\title{
Catalytic and Atom-Economic Intermolecular Amidoselenenylation of Alkenes
}

\author{
E Tang, ${ }^{*}$ Weilin Wang, Yinjiao Zhao, Meng Zhang, Xin Dai \\ Key Laboratory of Medicinal Chemistry for Natural Resources (Yunnan University) Ministry of \\ Education and School of Chemical Science and Technology, Yunnan University, 2 Green Lake North \\ Road, Kunming 650091, China, E-mail: tange@ynu.edu.cn
}

\section{Supporting Information}

Table of Contents

General Information.

Experimental procedures of products $3, \mathbf{4 a}, \mathbf{9 a}, \mathbf{5 a}, \mathbf{5} \mathbf{a}^{\prime}, \mathbf{5} \mathbf{s}$, and $\mathbf{5} \mathbf{s}^{\prime}$ .S1-S2

Analysis of the relative configuration of the products $( \pm)-3 \mathrm{c}$ and $( \pm)-3 \mathrm{~s}$.

Characterization of products $\mathbf{3}$.......

Characterization of products $4 \mathbf{a}, \mathbf{9 a}, \mathbf{5 a}, \mathbf{5} \mathbf{a}^{\prime}, \mathbf{5} \mathbf{s}$, and $\mathbf{5} \mathbf{s}^{\prime}$ S9-S11

X-ray structure of compound $\mathbf{3 o}$. $\mathrm{S} 11-\mathrm{S} 20$

References.

Copies of NMR Spectra for compounds S22-S45

Copies of ROESY Spectra for compounds $\mathbf{5 c}$ and $\mathbf{5 s}$ S46-S47 


\section{General Information}

All reactions were conducted using oven-dried glassware under an atmosphere of Nitrogen $\left(\mathrm{N}_{2}\right)$. Commercial grade reagents were purchased from Aladdin and Acros and used without further purification. All solvents were dried and distilled before use according to proper procedures. Alkenes $\mathbf{1 a},{ }^{[1]} \mathbf{1 f},{ }^{[1]} \mathbf{1 m},{ }^{[2]}$ 1n, ${ }^{[2]} \mathbf{1 0},{ }^{[2]} N$-phenylselenophthalimide (NPSP),${ }^{[3]}$ and $N$-phenylselenosuccinimide (NPSS) ${ }^{[4]}$ were prepared according to the literatures. The ${ }^{1} \mathrm{H}$ NMR and ${ }^{13} \mathrm{C}$ NMR spectra were recorded on Bruker Advance-400 or Bruker Advance-300. High Resolution Mass spectra (HRMS) were obtained by the ESI method from Agilent LC/MSD TOF instrument. HMBC, HSQC, and ROESY spectra were recorded on Bruker Advance-400. The infrared (IR) spectra were acquired on a FT-IR spectrometer.

\section{Experimental procedures of products 3:}

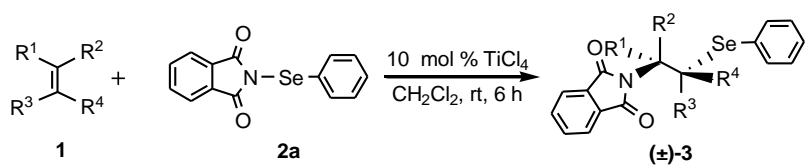

To a stirred solution of NPSP $(166.2 \mathrm{mg}, 0.55 \mathrm{mmol})$ in $\mathrm{CH}_{2} \mathrm{Cl}_{2}(5 \mathrm{~mL})$ at room temperature, $\mathrm{TiCl}_{4}(5.5 \mu \mathrm{L})$ was added. The reaction mixture was stirred for $5 \mathrm{~min}$ and the alkenes $(0.5 \mathrm{mmol})$ were added. The reaction mixture was stirred for another $6 \mathrm{~h}$ at this temperature. After the completion of the reaction, it was quenched with saturated aqueous $\mathrm{NaHCO}_{3}$ solution and the mixture was extracted with $\mathrm{CH}_{2} \mathrm{Cl}_{2}(3 \times 10 \mathrm{~mL})$. The organic layer was dried over anhydrous $\mathrm{MgSO}_{4}$ and filtered. After removal of the solvents under reduced pressure, the crude products were purified by flash chromatography on silica Gel (elution with petroleum ether/ethyl acetate: 10/1) to give the products 3 .

\section{Experimental procedures of products $4 a$ and 9a}

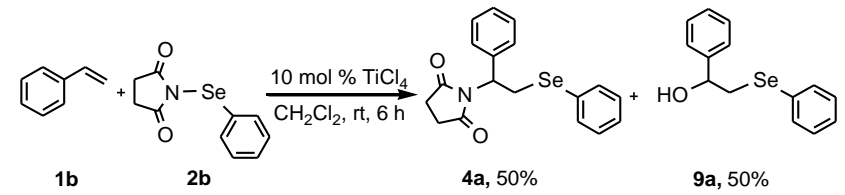

To a stirred solution of NPSS (139.8 mg, $0.55 \mathrm{mmol})$ in $\mathrm{CH}_{2} \mathrm{Cl}_{2}(5 \mathrm{~mL})$ at room temperature, $\mathrm{TiCl}_{4}(5.5 \mu \mathrm{L})$ was added. The reaction mixture was stirred for $5 \mathrm{~min}$ and styrene $(52.1 \mathrm{mg}, 0.5 \mathrm{mmol})$ were added. The reaction mixture was stirred for another $6 \mathrm{~h}$ at this temperature. After the completion of the reaction, it was quenched with saturated aqueous $\mathrm{NaHCO}_{3}$ solution and the mixture was extracted with $\mathrm{CH}_{2} \mathrm{Cl}_{2}(3 \times 10 \mathrm{~mL})$. The organic layer was dried over anhydrous $\mathrm{MgSO}_{4}$ and filtered. After removal of the solvents under reduced pressure, the crude products were purified by flash chromatography on silica Gel (elution with petroleum ether/ethyl acetate: 10/1) to give the products $4 \mathbf{a}(90 \mathrm{mg}, 50 \%)$ and $9 \mathbf{a}(69 \mathrm{mg}, 50 \%)$.

\section{Experimental procedures of product $4 \mathrm{a}$ from $9 \mathrm{a}^{[6]}$}

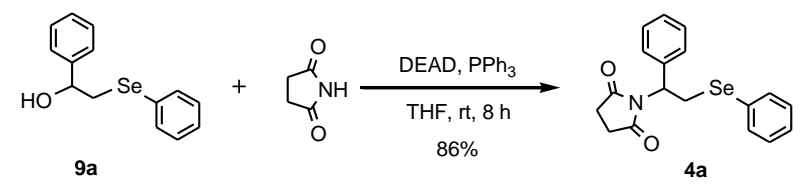

Triphenylphosphine $\left(\mathrm{Ph}_{3} \mathrm{P}, 57.7 \mathrm{mg}, 0.22 \mathrm{mmol}\right)$ and succinimide $(21.8 \mathrm{mg}, 0.22 \mathrm{mmol})$ were dissolved in THF $(5 \mathrm{~mL})$. To this mixture was added 1-phenyl-2-(phenylseleno)ethanol (9a, $55.4 \mathrm{mg}, 0.2 \mathrm{mmol})$ 
dissolved in THF ( $2 \mathrm{~mL}$ ), followed by the diethyl azodicarboxylate (DEAD) solution (40\% in toluene, 41.8 $\mathrm{mg}, 0.24 \mathrm{mmol})$. The mixture was stirred for $8 \mathrm{~h}$ at room temperature, before the solvent was removed under reduced pressure. The reaction mixture was then re-dissolved in $\mathrm{CH}_{2} \mathrm{Cl}_{2}(2 \mathrm{~mL}), \mathrm{K}_{2} \mathrm{CO}_{3}(0.2 \mathrm{M}, 0.8 \mathrm{~mL})$ added and the mixture stirred for $1 \mathrm{~h}$. The mixture was extracted with $\mathrm{CH}_{2} \mathrm{Cl}_{2}(3 \times 5 \mathrm{~mL})$ and the organic phase washed with brine and dried over anhydrous $\mathrm{MgSO}_{4}$. The solvent was removed under reduced pressure and the crude product was purified by silica-gel column chromatography (elution with petroleum ether/ethyl acetate: $10 / 1)$ to give the product $\mathbf{4 a}(62 \mathrm{mg}, 86 \%)$.

\section{Experimental procedures of products $5 \mathrm{a}, 5 \mathrm{a}^{\prime}, \mathbf{5 c}$, and $5 \mathrm{~s}$}
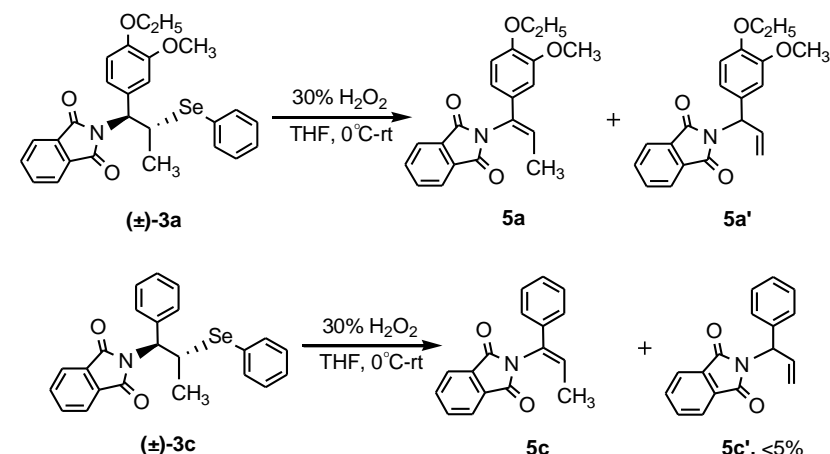

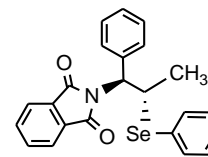

$( \pm)-3 s$

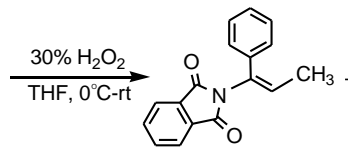

$5 s$

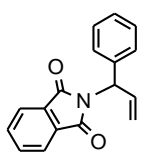

$5 c^{\prime},<5 \%$

$30 \%$ Aqueous $\mathrm{H}_{2} \mathrm{O}_{2}(0.5 \mathrm{~mL})$ solution was added to a solution of $3 \mathbf{a}$ or $\mathbf{3 c}$ or $\mathbf{3 s}(0.2 \mathrm{mmol})$ in $\mathrm{THF}(8 \mathrm{~mL})$ at $0^{\circ} \mathrm{C}$. The reaction mixture was stirred at $0^{\circ} \mathrm{C}$ for $1.5 \mathrm{~h}$ and then stirred at room temperature for 30 minutes. After the completion of the reaction, it was quenched with saturated aqueous $\mathrm{NaHSO}_{3}$ solution and the reaction mixture was extracted with $\mathrm{CH}_{2} \mathrm{Cl}_{2}(3 \times 10 \mathrm{~mL})$. The organic phase was dried over anhydrous $\mathrm{MgSO}_{4}$, filtered, and concentrated under reduced pressure. The residue was purified by flash chromatography on silica Gel (elution with petroleum ether/ethyl acetate: 10/1) to give $\mathbf{5 a}(54 \mathrm{mg}, 80 \%)$ and $\mathbf{5 a}$ ' (13 mg, 20\%) or 5c (49 $\mathrm{mg}, 93 \%)$ or $\mathbf{5 s}(47 \mathrm{mg}, 90 \%)$.

\section{Analysis of the relative configuration of the products ( \pm )-3c and $( \pm)-3 \mathrm{~s}$}

The products $( \pm)-\mathbf{3 c}$ and $( \pm)-3 \mathbf{s}$ were synthesized respectively from $(E)$ - $\beta$-methylstyphene (1a) and (Z)- $\beta$-methylstyphene (1s). The selenoxide syn-elimination of the compound $( \pm)-3 \mathbf{c}$ and $( \pm)$-3s afforded compounds $\mathbf{5 c}$ and $\mathbf{5 s}$. According to the mechanism of selenoxide elimination reaction, if a $\beta$-hydrogen atom is present, syn-elimination of selenoxide occurs easily to give the corresponding alkene (Scheme 1). ${ }^{[7]}$ Furthermore, according to the ROESY spectrum of $\mathbf{5 c}$, the correlation of $\delta_{\mathrm{H}}(7.32)$ and $\delta_{\mathrm{H}}(6.51)$, together with the ${ }^{1} \mathrm{H}$ NMR, and ${ }^{13} \mathrm{C}$ NMR spectra, the following structure of compound $\mathbf{5 c}$ can be deduced as $(Z)-2-[$ 1-phenyl-1-propenyl]-1H-isoindole-1,3(2H)-dione. Therefore, the alkene $\mathbf{5 c}$ was synthesized from (E)- $\beta$-methylstyphene (1a) via anti-addition with NPSP and syn-elimination of selenoxide, whereas the alkene 5s was obtained from (Z)- $\beta$-methylstyphene (1s) via anti-addition with NPSP and syn-elimination of selenoxide because of no correlation of $\delta_{\mathrm{H}}(7.36)$ and $\delta_{\mathrm{H}}(5.98)$ in the ROESY spectrum of $\mathbf{5} \mathbf{s}$. Therefore, the reaction of $(E)-\beta$-methylstyphene $(\mathbf{1 a})$ produces $(1 S, 2 S)$ and $(1 R, 2 R)$ adducts $( \pm)-3 \mathbf{c}$, and the reaction of $(Z)-\beta$-methylstyphene (1s) produces $(1 S, 2 R)$ and $(1 R, 2 S)$ adducts $( \pm)-3 \mathbf{s}$ (Scheme 2 and 3$)$. 


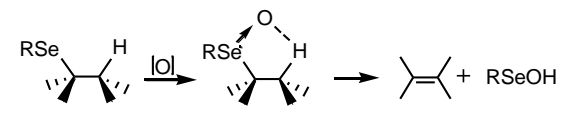

Scheme 1. The mechanism of selenoxide elimination reaction

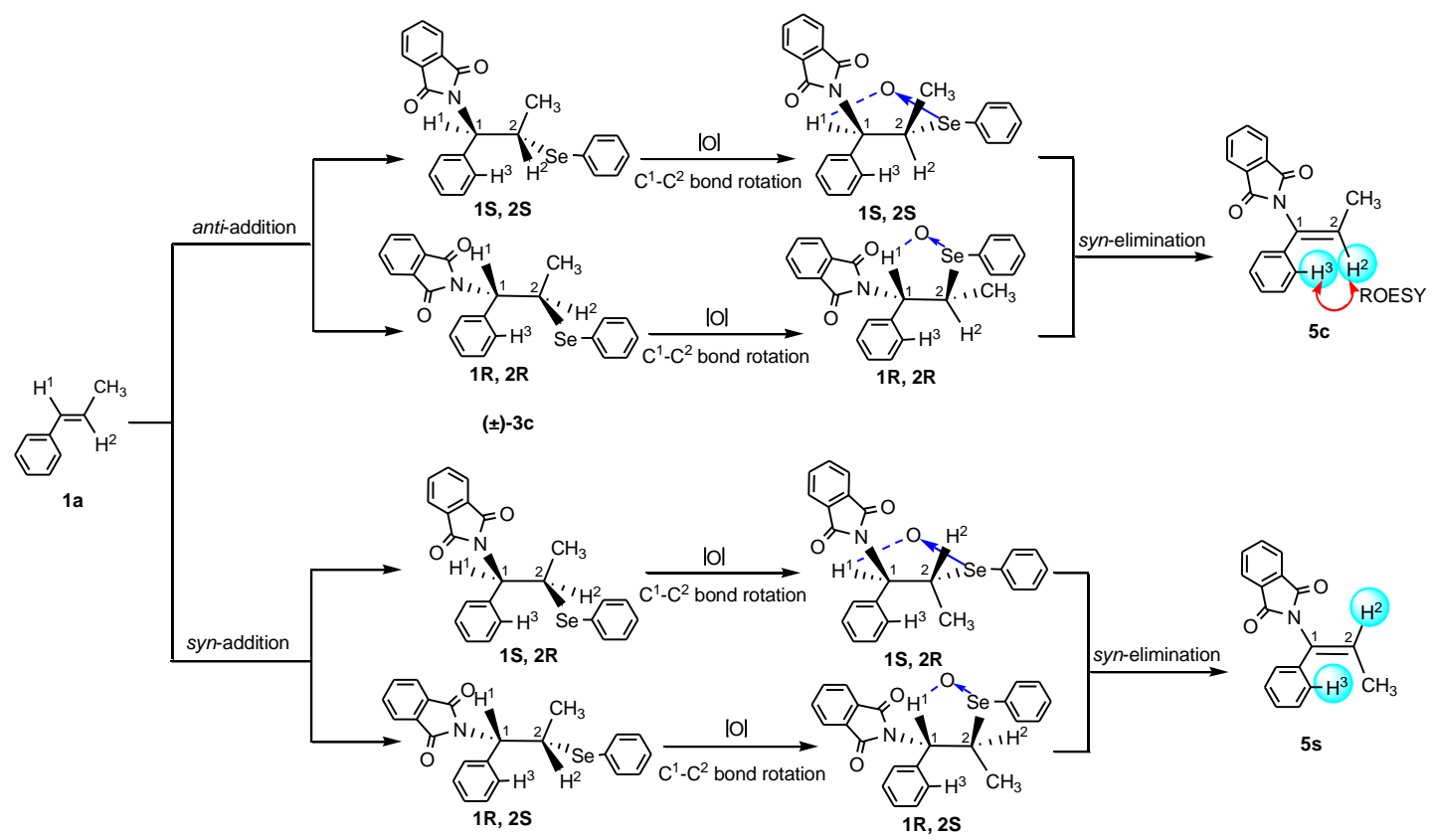

Scheme 2. Analysis of the relative configuration of the product $( \pm)-3 c$

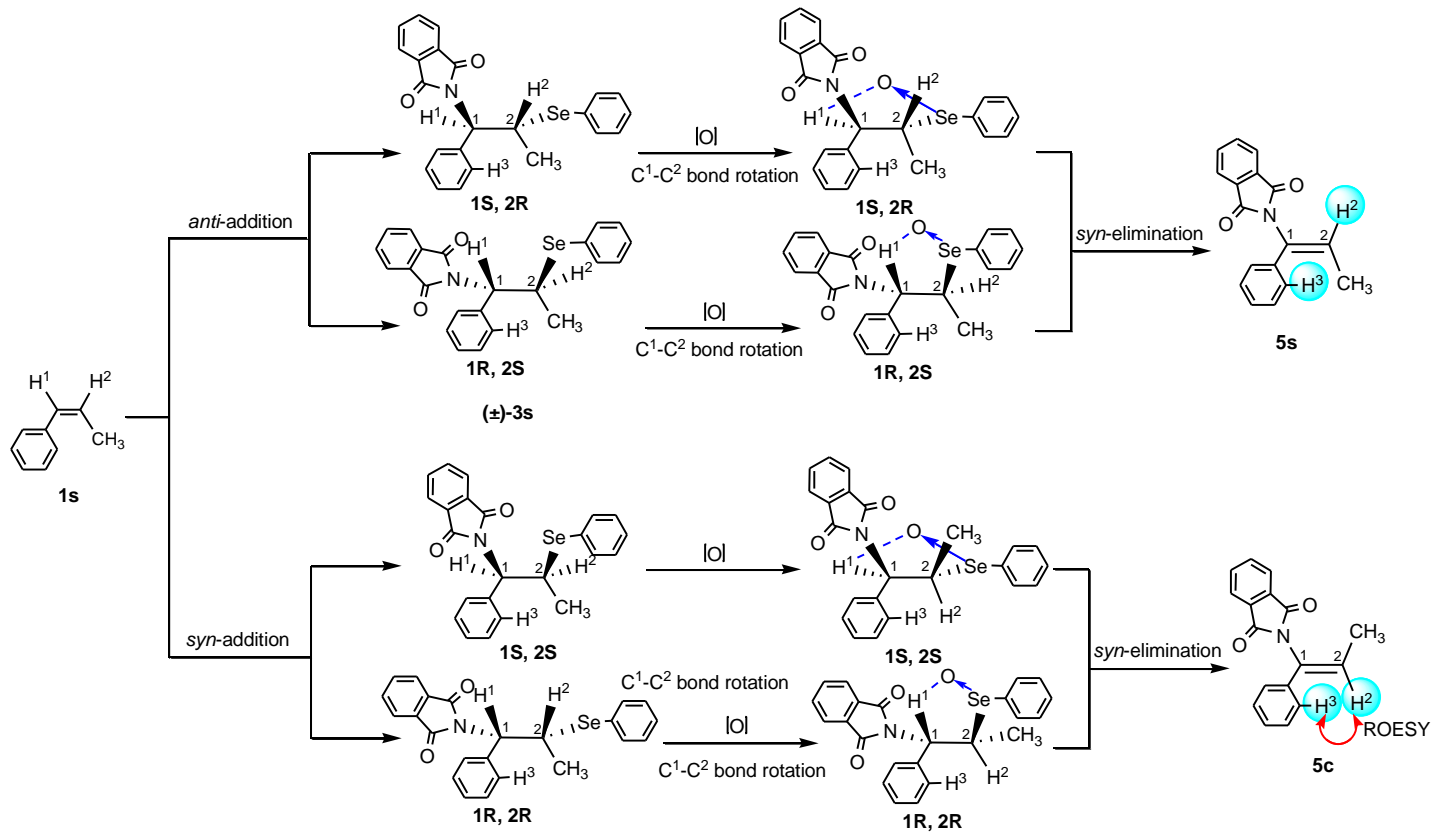

Scheme 3. Analysis of the relative configuration of the product ( \pm )-3s 


\section{Characterization of products 3}

2-[1-(3'-Methoxy-4'-ethoxy)phenyl-2-(phenylseleno)propyl]-1H-isoindole-1,3(2H)-dione $( \pm 3 a)$

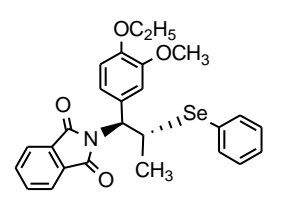

Yellow viscous liquid.

Yield: $230 \mathrm{mg}, 93 \%$.

${ }^{1}$ H NMR $\left(300 \mathrm{MHz}, \mathrm{CDCl}_{3}\right): \delta$ 7.79-7.50 (m, 2H), 7.70-7.66 (m, 2H), 7.43-7.40 $(\mathrm{m}, 2 \mathrm{H}), 7.28-7.19(\mathrm{~m}, 3 \mathrm{H}), 7.12-7.10(\mathrm{~m}, 2 \mathrm{H}), 6.75(\mathrm{~d}, J=8.7 \mathrm{~Hz}, 1 \mathrm{H}), 5.23(\mathrm{~d}, J$ $=12.0 \mathrm{~Hz}, 1 \mathrm{H}), 4.67-4.56(\mathrm{~m}, 1 \mathrm{H}), 4.07(\mathrm{q}, J=6.9,14.1 \mathrm{~Hz}, 2 \mathrm{H}), 3.79(\mathrm{~s}, 3 \mathrm{H})$, $1.44(\mathrm{t}, J=7.2 \mathrm{~Hz}, 6 \mathrm{H})$.

${ }^{13} \mathrm{C}$ NMR $\left(75 \mathrm{MHz}, \mathrm{CDCl}_{3}\right): \delta$ 168.2, 149.0, 148.3, 136.1, 134.1, 131.8, 131.0, 128.9, 128.4, 128.0, 123.4, 121.9, 112.5, 112.1, 64.3, 61.4, 55.9, 38.9, 20.6, 14.9 .

IR $\left(\mathrm{KBr}, \mathrm{cm}^{-1}\right): 2974,2921,1768,1709,1597,1514,1462,1377,1328,1262$, 1144, 1079, 1038, 731.

HRMS $\left(\mathrm{ESI}^{+}\right)$: calcd for $\left[\mathrm{C}_{26} \mathrm{H}_{25} \mathrm{NO}_{4} \mathrm{NaSe}\right]^{+}:$518.0846, found 518.0847.

2-[1-Phenyl-2-(phenylseleno)ethyl ]-1H-isoindole-1,3(2H)-dione (3b)

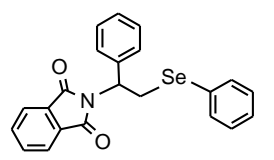

Yellow viscous liquid.

Yield: $142 \mathrm{mg}, 70 \%$.

${ }^{1} \mathbf{H}$ NMR $\left(300 \mathrm{MHz}, \mathrm{CDCl}_{3}\right): \delta$ 7.78-7.73 (m, 2H), 7.70-7.66 (m, 2H), 7.52-7.48 (m, 4H), 7.34-7.16 (m, 6H), $5.51(\mathrm{dd}, J=5.1,11.4 \mathrm{~Hz}, 1 \mathrm{H}), 4.27$ (dd, $J=11.4,12.9$ $\mathrm{Hz}, 1 \mathrm{H}), 3.55$ (dd, $J=5.1,13.2 \mathrm{~Hz}, 1 \mathrm{H}) \mathrm{ppm}$.

${ }^{13}$ C NMR (75 MHz, $\left.\mathrm{CDCl}_{3}\right): \delta 168.3,139.3,134.3,134.0,132.0,129.2,128.8$, 128.3, 128.1, 127.7, 123.4, 55.7, $29.6 \mathrm{ppm}$.

IR $\left(\mathrm{KBr}, \mathrm{cm}^{-1}\right):$ 3058, 2922, 2855, 1769, 1711, 1597, 1468, 1379, 1180, 1078, 718.

HRMS $\left(\mathrm{ESI}^{+}\right)$: calcd for $\left[\mathrm{C}_{22} \mathrm{H}_{17} \mathrm{NO}_{2} \mathrm{NaSe}\right]^{+}: 430.0322$, found 430.0322 .

(E)-2-[1-Phenyl-2-(phenylseleno)propyl]-1H-isoindole-1,3(2H)-dione $( \pm 3 c)$

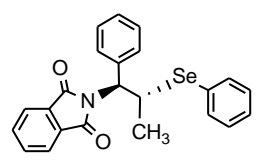

Colorless solid.

Mp: $93.6-94.1^{\circ} \mathrm{C}$.

Yield: $174 \mathrm{mg}, 83 \%$.

${ }^{1} \mathbf{H}$ NMR $\left(300 \mathrm{MHz}, \mathrm{CDCl}_{3}\right): \delta$ 7.79-7.75 (m, 2H), 7.69-7.65 (m, 2H), 7.61-7.58 (m, 2H), 7.45-7.42 (m, 2H), 7.32-7.20 (m, 6H), 5.29 (d, J=12 Hz, 1H), 4.71-4.61 $(\mathrm{m}, 1 \mathrm{H}), 1.45(\mathrm{~d}, J=6.6 \mathrm{~Hz}, 3 \mathrm{H}) \mathrm{ppm}$.

${ }^{13}$ C NMR (75 MHz, $\left.\mathrm{CDCl}_{3}\right): \delta 168.1,138.5,136.3,134.2,131.7,129.3,129.0$, 128.6, 128.4, 128.2, 127.9, 123.4, 61.1. 37.9, $20.6 \mathrm{ppm}$.

IR $\left(\mathrm{KBr}, \mathrm{cm}^{-1}\right): 3059,2923,2855,1769,1711,1610,1458,1378,1174,1180$, 1012, 720 .

HRMS $\left(\mathrm{ESI}^{+}\right)$: calcd for $\left[\mathrm{C}_{23} \mathrm{H}_{19} \mathrm{NO}_{2} \mathrm{NaSe}\right]^{+}: 444.0479$, found 444.0468 .

2-[1-(4'-Methyl)phenyl-2-(phenylseleno)propyl]-1H-isoindole-1,3(2H)-dione (3d)

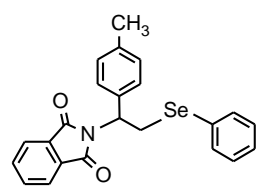

Yellow viscous liquid.

Yield: $153 \mathrm{mg}, 73 \%$.

${ }^{1} \mathbf{H}$ NMR $\left(300 \mathrm{MHz}, \mathrm{CDCl}_{3}\right): \delta$ 7.76-7.72 (m, 2H), 7.69-7.64 (m, 2H), 7.51-7.47 $(\mathrm{m}, 2 \mathrm{H}), 7.39(\mathrm{~d}, J=8.1 \mathrm{~Hz}, 2 \mathrm{H}), 7.17-7.10(\mathrm{~m}, 5 \mathrm{H}), 5.48(\mathrm{dd}, J=4.8,11.1 \mathrm{~Hz}$, $1 \mathrm{H}), 4.24$ (q, $J=11.4,12.9 \mathrm{~Hz}, 1 \mathrm{H}), 3.54(\mathrm{dd}, J=4.8,12.9 \mathrm{~Hz}, 1 \mathrm{H}), 2.30$ (s, 3H) ppm.

${ }^{13}$ C NMR $\left(75 \mathrm{MHz}, \mathrm{CDCl}_{3}\right): \delta 168.3,138.1,136.3,134.2,134.0,131.9,129.4$, 
$129.2,128.7,128.0,127.6,123.3,55.4,29.6,21.2 \mathrm{ppm}$.

IR $\left(\mathrm{KBr}, \mathrm{cm}^{-1}\right): 2923,1770,1466,1379,1181,1078,726$.

HRMS $\left(\mathrm{ESI}^{+}\right)$: calcd for $\left[\mathrm{C}_{23} \mathrm{H}_{19} \mathrm{NO}_{2} \mathrm{NaSe}\right]^{+}: 444.0479$, found 444.0469 .

2-[1-(4'-Methoxy)phenyl-2(phenylseleno)propyl]-1H-isoindole-1,3(2H)-dione (3e)

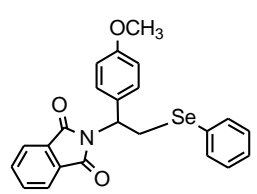

Yellow viscous liquid.

Yield: $164 \mathrm{mg}, 75 \%$.

${ }^{1} \mathbf{H}$ NMR $\left(300 \mathrm{MHz}, \mathrm{CDCl}_{3}\right): \delta$ 7.76-7.73 (m, 2H), 7.67-7.65 (m, 2H), 7.51-7.42 (m, 4H), $7.16(\mathrm{t}, J=3 \mathrm{~Hz}, 3 \mathrm{H}), 6.83(\mathrm{~d}, J=8.7 \mathrm{~Hz}, 2 \mathrm{H}), 5.47$ (dd, $J=5.1,11.1$ $\mathrm{Hz}, 1 \mathrm{H}), 4.23$ (dd, $J=11.1,12.9 \mathrm{~Hz}, 1 \mathrm{H}), 3.76$ (s, 3H), 3.54 (dd, $J=5.1,12.9 \mathrm{~Hz}$, 1H) $\mathrm{ppm}$.

${ }^{13}$ C NMR $\left(75 \mathrm{MHz}, \mathrm{CDCl}_{3}\right): \delta 168.3,159.5,134.2,134.0,131.9,131.4,129.4$, 129.2, 127.6, 123.3, 114.0, 55.4, 55.1, 29.7 ppm.

IR $\left(\mathrm{KBr}, \mathrm{cm}^{-1}\right): 2928,1769,1710,1610,1512,1465,1379,1250,1078,1035$, 725 .

HRMS $\left(\mathrm{ESI}^{+}\right)$: calcd for $\left[\mathrm{C}_{23} \mathrm{H}_{19} \mathrm{NO}_{3} \mathrm{NaSe}\right]^{+}: 460.0428$, found 460.0426 .

2-[1-(3', 4'-Diethoxy)phenyl-2-(phenylseleno)propyl]-1H-isoindole-1,3(2H)-dione $( \pm 3 f)$

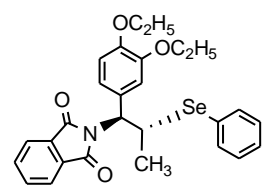

Yellow viscous liquid.

Yield: $229 \mathrm{mg}, 90 \%$.

${ }^{1}$ H NMR $\left(300 \mathrm{MHz}, \mathrm{CDCl}_{3}\right): \delta$ 7.78-7.74 (m, 2H), 7.69-7.64 (m, 2H), 7.44-7.41 $(\mathrm{m}, 2 \mathrm{H}), 7.28-7.18(\mathrm{~m}, 3 \mathrm{H}), 7.14-7.07(\mathrm{~m}, 2 \mathrm{H}), 6.76(\mathrm{~d}, J=8.4 \mathrm{~Hz}, 1 \mathrm{H}), 5.22(\mathrm{~d}, J$ $=12.0 \mathrm{~Hz}, 1 \mathrm{H}), 4.66-4.55(\mathrm{~m}, 1 \mathrm{H}), 4.09-4.00(\mathrm{~m}, 4 \mathrm{H}), 1.45-1.39(\mathrm{~m}, 9 \mathrm{H}) \mathrm{ppm}$.

${ }^{13}$ C NMR $\left(75 \mathrm{MHz}, \mathrm{CDCl}_{3}\right): \delta 168.2,148.7,148.5,136.2,134.1,131.8,131.1$, $128.9,128.4,128.0,123.4,121.9$, 114.4, 112.9, 64.53, 64.50, 61.3, 38.8, 20.6, 15.0, $14.9 \mathrm{ppm}$.

IR $\left(\mathrm{KBr}, \mathrm{cm}^{-1}\right): 2974,1768,1709,1513,1381,1047,883,730$.

HRMS $\left(\mathrm{ESI}^{+}\right.$): calcd for $\left[\mathrm{C}_{27} \mathrm{H}_{27} \mathrm{NO}_{4} \mathrm{NaSe}\right]^{+}:$532.1003, found 532.0993.

2-[1-( 4'-Methoxy)phenyl-2-(phenylseleno)propyl ]-1H-isoindole-1,3(2H)-dione $( \pm 3 g)$

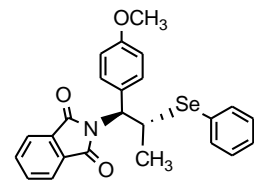

Yellow viscous liquid.

Yield: $194 \mathrm{mg}, 86 \%$.

${ }^{1}$ H NMR $\left(300 \mathrm{MHz}, \mathrm{CDCl}_{3}\right): \delta$ 7.78-7.74 (m, 2H), 7.68-7.64 (m, 2H), $7.52(\mathrm{~d}, J=$ $9.0 \mathrm{~Hz}, 2 \mathrm{H}), 7.46-7.43(\mathrm{dd}, J=7.8,1.2 \mathrm{~Hz}, 2 \mathrm{H}), 7.31-7.18(\mathrm{~m}, 3 \mathrm{H}), 6.80(\mathrm{~d}, J=$ $8.7 \mathrm{~Hz}, 2 \mathrm{H}), 5.25(\mathrm{~d}, J=12 \mathrm{~Hz}, 1 \mathrm{H}), 4.70-4.59(\mathrm{~m}, 1 \mathrm{H}), 3.77$ (s, 3H), $1.43(\mathrm{~d}, J=$ $6.9 \mathrm{~Hz}, 3 \mathrm{H}) \mathrm{ppm}$.

${ }^{13}$ C NMR $\left(75 \mathrm{MHz}, \mathrm{CDCl}_{3}\right): \delta 168.2,159.5,136.3,134.2,131.8,130.7,130.5$, 129.0, 128.1, 123.4, 113.8, 60.7, 55.3, 38.4, $20.6 \mathrm{ppm}$.

IR $\left(\mathrm{KBr}, \mathrm{cm}^{-1}\right): 2967,2921,1768,1710,1610,1513,1460,1379,1249,1178$, 1082, 1033, 727.

HRMS $\left(\mathrm{ESI}^{+}\right)$: calcd for $\left[\mathrm{C}_{24} \mathrm{H}_{21} \mathrm{NO}_{3} \mathrm{NaSe}\right]^{+}: 474.0584$, found 474.0578.

2-[1-(4'-Chloro)phenyl-2-(phenylseleno)propyl ]-1H-isoindole-1,3(2H)-dione (3h)

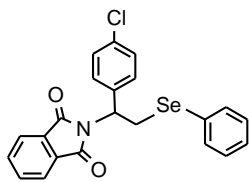

Yellow solid.

Mp: $67.4-68.2^{\circ} \mathbf{C}$.

Yield: $165 \mathrm{mg}, 75 \%$.

${ }^{1}$ H NMR $\left(300 \mathrm{MHz}, \mathrm{CDCl}_{3}\right): \delta$ 7.77-7.73 (m, 2H), 7.71-7.66 (m, 2H), 7.50-7.47 (m, 2H), 7.44-7.41 (m, 2H), 7.28-7.25 (m, 2H), 7.18-7.16 (m, 3H), 5.47 (dd, $J=$ 
5.4, $11.1 \mathrm{~Hz}, 1 \mathrm{H}), 4.18(\mathrm{dd}, J=10.8,12.9 \mathrm{~Hz}, 1 \mathrm{H}), 3.53(\mathrm{dd}, J=5.4,12.9 \mathrm{~Hz}, 1 \mathrm{H})$ ppm.

${ }^{13}$ C NMR $\left(75 \mathrm{MHz}, \mathrm{CDCl}_{3}\right): \delta 168.2,137.7,134.4,134.2,134.1,131.8,129.6$, $129.3,129.0,128.5,127.8,123.5,55.0,29.3 \mathrm{ppm}$.

IR $\left(\mathrm{KBr}, \mathrm{cm}^{-1}\right): 2925,1770,1712,1485,1379,1084,726$.

HRMS $\left(\mathrm{ESI}^{+}\right)$: calcd for $\left[\mathrm{C}_{22} \mathrm{H}_{16} \mathrm{ClKNO}_{2} \mathrm{Se}^{+}:\right.$: 479.9672, found 479.9667 .

2-[1-(4'-Fluoro)phenyl-2-(phenylseleno)propyl]-1H-isoindole-1,3(2H)-dione (3i)

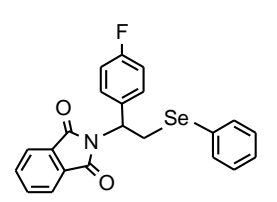

Yellow viscous liquid.

Yield: $151 \mathrm{mg}, 71 \%$.

${ }^{1}$ H NMR $\left(300 \mathrm{MHz}, \mathrm{CDCl}_{3}\right): \delta$ 7.77-7.73 (m, 2H), 7.70-7.66 (m, 2H), 7.50-7.45 (m, 4H), 7.19-7.16 (m, 3H), 6.98 (t, $J=8.7 \mathrm{~Hz}, 2 \mathrm{H}), 5.48$ (dd, $J=5.4,11.1 \mathrm{~Hz}$, $1 \mathrm{H}), 4.20(\mathrm{dd}, J=11.1,12.9 \mathrm{~Hz}, 1 \mathrm{H}), 3.53(\mathrm{dd}, J=5.1,12.9 \mathrm{~Hz}, 1 \mathrm{H}) \mathrm{ppm}$.

${ }^{13}$ C NMR (75 MHz, $\left.\mathrm{CDCl}_{3}\right): \delta 168.2,162.6(\mathrm{~d}, J=246 \mathrm{~Hz}), 135.1,134.3,134.1$, $131.9,130.0$ (d, $J=8.25 \mathrm{~Hz}), 129.2,128.5,127.8,123.4,115.7$ (d, $J=21.75 \mathrm{~Hz})$, 55.0, $29.5 \mathrm{ppm}$.

IR (KBr, cm $\left.{ }^{-1}\right):$ 2929, 1770, 1711, 1605, 1510, 1378, 1228, 1079, 834, 725.

HRMS $\left(\mathrm{ESI}^{+}\right)$: calcd for $\left[\mathrm{C}_{22} \mathrm{H}_{16} \mathrm{FKNO}_{2} \mathrm{Se}\right]^{+}:$: 463.9967, found 463.9966.

2-[1-(4'-Bromo)phenyl-2-(phenylseleno)propyl]-1H-isoindole-1,3(2H)-dione (3j)

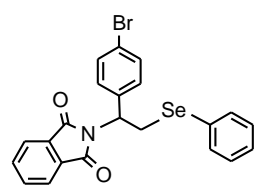

Yellow viscous liquid.

Yield: $184 \mathrm{mg}, 76 \%$.

${ }^{1}$ H NMR (300 MHz, $\mathrm{CDCl}_{3}$ ): $\delta$ 7.78-7.75 (m, 2H), 7.70-7.67 (m, 2H), 7.50-7.35 $(\mathrm{m}, 6 \mathrm{H}), 7.17(\mathrm{t}, J=3.2 \mathrm{~Hz}, 3 \mathrm{H}), 5.45(\mathrm{dd}, J=5.1,10.8 \mathrm{~Hz}, 1 \mathrm{H}), 4.18(\mathrm{dd}, J=$ $11.1,13.2 \mathrm{~Hz}, 1 \mathrm{H}), 3.53(\mathrm{dd}, J=5.4,12.9 \mathrm{~Hz}, 1 \mathrm{H}) \mathrm{ppm}$.

${ }^{13}$ C NMR (75 MHz, $\left.\mathrm{CDCl}_{3}\right): \delta 168.2,138.2,134.4,134.2,131.9,131.8,129.9$, $129.3,128.5,127.8,123.5,122.4,55.1,29.3 \mathrm{ppm}$.

IR $\left(\mathrm{KBr}, \mathrm{cm}^{-1}\right): 2922,1770,1712,1480,1380,1077,823,726$.

HRMS $\left(\mathrm{ESI}^{+}\right)$: calcd for $\left[\mathrm{C}_{22} \mathrm{H}_{16} \mathrm{BrKNO}_{2} \mathrm{Se}\right]^{+}:$523.9167, found 523.9158.

2-[2-phenyl-1-phenylseleo-2-propyl]-1H-isoindole-1,3(2H)-dione (3k)

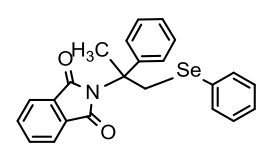

Colorless viscous liquid.

Yield: $36 \mathrm{mg}, 17 \%$.

${ }^{1} \mathbf{H}$ NMR $\left(300 \mathrm{MHz}, \mathrm{CDCl}_{3}\right): \delta$ 7.69-7.62 (m, 4H), 7.48-7.37 (m, 2H), 7.35-7.19 $(\mathrm{m}, 5 \mathrm{H}), 7.07-7.02(\mathrm{~m}, 3 \mathrm{H}), 4.51(\mathrm{~d}, J=12.6 \mathrm{~Hz}, 1 \mathrm{H}), 3.60(\mathrm{~d}, J=12.6 \mathrm{~Hz}, 1 \mathrm{H})$, $2.15(\mathrm{~s}, 3 \mathrm{H}) \mathrm{ppm}$.

${ }^{13}$ C NMR $\left(75 \mathrm{MHz}, \mathrm{CDCl}_{3}\right): \delta 169.3,145.5,133.9,133.7,131.8,130.3,128.9$, $128.7,127.3,127.2,124.8,123.1,65.1,40.3,27.9$ ppm.

IR $\left(\mathrm{KBr}, \mathrm{cm}^{-1}\right): 1771,1710,1599,1449,1363,1317,1196,1059,717$.

HRMS (ESI ${ }^{+}$): calcd for $\left[\mathrm{C}_{23} \mathrm{H}_{19} \mathrm{NO}_{2} \mathrm{NaSe}^{+}:\right.$444.0479, found 444.0470 .

2-[3-Methoxy-1-phenyl-2-(phenylseleno)-1-propyl]-1H-isoindole-1,3(2H)-dione $( \pm 3 \mathrm{~m})$

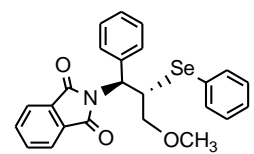

Colorless solid.

Mp: $96.6-97.1^{\circ} \mathrm{C}$.

Yield: $189 \mathrm{mg}, 84 \%$.

${ }^{1}$ H NMR $\left(300 \mathrm{MHz}, \mathrm{CDCl}_{3}\right): \delta$ 7.79-7.77 (m, 2H), 7.68-7.65 (m, 2H), 7.56-7.53 $(\mathrm{m}, 2 \mathrm{H}), 7.35-7.32(\mathrm{~m}, 2 \mathrm{H}), 7.28-7.15(\mathrm{~m}, 6 \mathrm{H}), 5.59(\mathrm{~d}, J=12.3 \mathrm{~Hz}, 1 \mathrm{H})$, 4.70-4.63 (m, 1H), $3.71(\mathrm{t}, J=4.2 \mathrm{~Hz}, 2 \mathrm{H}), 3.17$ (s, 3H) ppm. 
${ }^{13} \mathbf{C}$ NMR $\left(75 \mathrm{MHz}, \mathrm{CDCl}_{3}\right): \delta 168.2,138.2,135.6,134.1,132.0,129.6,129.0$, $128.7,128.4,128.3,128.0,123.4,74.6,59.3,57.6,44.1 \mathrm{ppm}$.

IR $\left(\mathrm{KBr}, \mathrm{cm}^{-1}\right): 2922,1770,1712,1605,1462,1081,721$.

HRMS (ESI ${ }^{+}$): calcd for $\left[\mathrm{C}_{24} \mathrm{H}_{21} \mathrm{NO}_{3} \mathrm{NaSe}\right]^{+}$: 474.0584 , found 474.0573 .

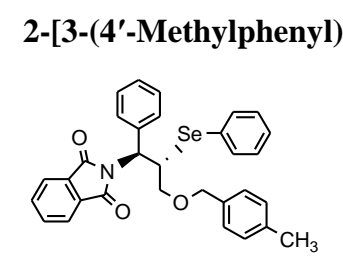

methoxy-1-phenyl-2-(phenylseleno)propyl]-1H-isoindole-1,3(2H)-dione $( \pm 3 n)$

Yellow viscous liquid.

Yield: $235 \mathrm{mg}, 87 \%$.

${ }^{1} \mathbf{H}$ NMR $\left(300 \mathrm{MHz}, \mathrm{CDCl}_{3}\right): \delta$ 7.68-7.64 (m, 2H), 7.63-7.59 (m, 2H), 7.54-7.51 $(\mathrm{m}, 2 \mathrm{H}), 7.30-7.11(\mathrm{~m}, 8 \mathrm{H}), 6.97(\mathrm{~d}, J=7.8 \mathrm{~Hz}, 2 \mathrm{H}), 6.87(\mathrm{~d}, J=7.8 \mathrm{~Hz}, 2 \mathrm{H})$, $5.62(\mathrm{~d}, J=12.3 \mathrm{~Hz}, 1 \mathrm{H}), 4.68(\mathrm{dt}, J=3.9,12.3 \mathrm{~Hz}, 1 \mathrm{H}), 4.40(\mathrm{~d}, J=11.7 \mathrm{~Hz}$, $1 \mathrm{H}), 4.23(\mathrm{~d}, J=11.7 \mathrm{~Hz}, 1 \mathrm{H}), 3.76$ (d, $J=3.9 \mathrm{~Hz}, 2 \mathrm{H}), 2.17$ (s, $3 \mathrm{H}) \mathrm{ppm}$.

${ }^{13}$ C NMR $\left(75 \mathrm{MHz}, \mathrm{CDCl}_{3}\right): \delta 168.2,138.4,137.1,135.5,134.5,133.8,131.9$, $129.5,128.9,128.9,128.4,128.2,128.1,127.9,123.2,73.3,71.7,57.7,44.5,21.2$ ppm.

IR $\left(\mathrm{KBr}, \mathrm{cm}^{-1}\right): 2923,1771,1712,1458,1385,1080,720$.

HRMS (ESI ${ }^{+}$): calcd for $\left[\mathrm{C}_{31} \mathrm{H}_{27} \mathrm{KNO}_{3} \mathrm{Se}^{+}: 580.0793\right.$, found 580.0791 .

2-[3-(2'-Methylphenyl)methoxy-1-phenyl-2-(phenylseleno)propyl]-1H-isoindole-1,3(2H)-dione $( \pm 30)$

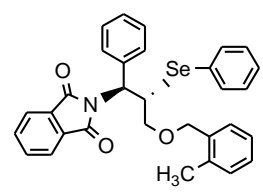

Colorless solid.

Mp: $119.3-120.1^{\circ} \mathrm{C}$.

Yield: $230 \mathrm{mg}, 85 \%$.

${ }^{1} \mathbf{H}$ NMR $\left(300 \mathrm{MHz}, \mathrm{CDCl}_{3}\right): \delta$ 7.65-7.61 (m, 2H), 7.59-7.51 (m, 4H), 7.30-7.11 (m, 8H), $7.03(\mathrm{~d}, J=7.5 \mathrm{~Hz}, 1 \mathrm{H}), 6.98-6.87(\mathrm{~m}, 3 \mathrm{H}), 5.60(\mathrm{~d}, J=12.0 \mathrm{~Hz}, 1 \mathrm{H})$, $4.71(\mathrm{dt}, J=3.9,12.3 \mathrm{~Hz}, 1 \mathrm{H}), 4.38(\mathrm{~d}, J=12 \mathrm{~Hz}, 1 \mathrm{H}), 4.29(\mathrm{~d}, J=11.7 \mathrm{~Hz}, 1 \mathrm{H})$, $3.83(\mathrm{~d}, J=3.9 \mathrm{~Hz}, 2 \mathrm{H}), 2.19$ (s, 3H) ppm.

${ }^{13}$ C NMR $\left(75 \mathrm{MHz}, \mathrm{CDCl}_{3}\right)$ : $\delta 168.1,138.4,136.6,135.6,135.5,133.8,131.8$, 130.1, 129.5, 128.9, 128.8, 128.7, 128.4, 128.2, 127.9, 127.6, 125.5, 123.2, 72.3, $71.6,57.8,44.5,18.9 \mathrm{ppm}$.

IR $\left(\mathrm{KBr}, \mathrm{cm}^{-1}\right): 2921,2848,1770,1710,1464,1378,1084,722$.

HRMS (ESI ${ }^{+}$): calcd for $\left[\mathrm{C}_{31} \mathrm{H}_{27} \mathrm{NO}_{3} \mathrm{NaSe}\right]^{+}: 564.1054$, found 564.1049.

2-(2-Phenylselenocyclohexyl)-1 $H$-Isoindole-1,3(2H)-dione $( \pm 3 p)$

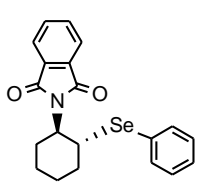

Colorless solid.

Mp: $95.9-96.2^{\circ} \mathrm{C}$.

Yield: $133 \mathrm{mg}, 69 \%$.

${ }^{1}$ H NMR (300 MHz, $\mathrm{CDCl}_{3}$ ): $\delta$ 7.70-7.63 (m, 4H), 7.42-7.39 (m, 2H), 7.03-7.00 $(\mathrm{m}, 3 \mathrm{H}), 4.26-4.16(\mathrm{~m}, 1 \mathrm{H}), 4.12-4.03(\mathrm{~m}, 1 \mathrm{H}), 2.34-2.16(\mathrm{~m}, 2 \mathrm{H}), 1.85-1.81(\mathrm{~m}$, $2 \mathrm{H}), 1.75-1.72(\mathrm{~m}, 1 \mathrm{H}), 1.56-1.29(\mathrm{~m}, 3 \mathrm{H}) \mathrm{ppm}$.

${ }^{13}$ C NMR $\left(75 \mathrm{MHz}, \mathrm{CDCl}_{3}\right): \delta 168.3,135.5,133.7,131.9,128.7,127.6,127.5$, 123.2, 56.0, 44.2, 34.3, 31.2, 26.8, $25.6 \mathrm{ppm}$.

IR $\left(\mathrm{KBr}, \mathrm{cm}^{-1}\right): 2937,2856,1757,1708,1455,1375,1183,1120,1065,715$.

HRMS (ESI ${ }^{+}$): calcd for $\left[\mathrm{C}_{20} \mathrm{H}_{19} \mathrm{NO}_{2} \mathrm{NaSe}\right]^{+}: 408.0479$, found 408.0474 .

2-(2-Phenylseleno-1,2,3,4-tetrahydro-1-naphthalenyl)-1 $H$-Isoindole-1,3(2H)-dione $( \pm 3 q)$ 


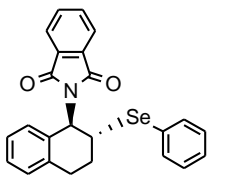

Brown viscous liquid.

Yield: $177 \mathrm{mg}, 82 \%$.

${ }^{1}$ H NMR $\left(300 \mathrm{MHz}, \mathrm{CDCl}_{3}\right): \delta$ 7.77-7.73 $(\mathrm{m}, 2 \mathrm{H}), 7.71-7.66(\mathrm{~m}, 2 \mathrm{H})$, 7.56-7.53 $(\mathrm{m}, 2 \mathrm{H}), 7.12-7.01(\mathrm{~m}, 6 \mathrm{H}), 6.89(\mathrm{~d}, J=7.8 \mathrm{~Hz}, 1 \mathrm{H}), 5.63(\mathrm{~d}, J=11.8 \mathrm{~Hz}, 1 \mathrm{H})$, 4.36- $4.27(\mathrm{~m}, 1 \mathrm{H}), 3.22-3.11(\mathrm{~m}, 1 \mathrm{H}), 2.91-2.84(\mathrm{~m}, 1 \mathrm{H}), 2.55-2.47(\mathrm{~m}, 1 \mathrm{H})$, 2.04-1.89 (m, 1H) ppm.

${ }^{13}$ C NMR (75 MHz, $\left.\mathrm{CDCl}_{3}\right): \delta 168.2,136.7,135.8,134.7,134.0,131.9,129.0$, $128.9,127.8,127.3,127.0,126.6,125.9,123.4,55.8,41.7,31.1,30.5$ ppm.

IR $\left(\mathrm{KBr}, \mathrm{cm}^{-1}\right): 2925,1769,1711,1609,1472,1380,1091,729$.

HRMS $\left(\mathrm{ESI}^{+}\right)$: calcd for $\left[\mathrm{C}_{24} \mathrm{H}_{19} \mathrm{NO}_{2} \mathrm{NaSe}\right]^{+}: 456.0479$, found 456.0478 .

2-(1-Phenylseleno-2-octyl)- $1 H$-isoindole-1,3(2H)-dione (3r)

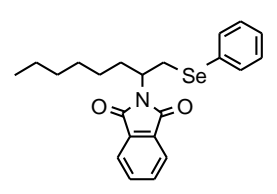

Colorless viscous liquid.

Yield: $108 \mathrm{mg}, 52 \%$.

${ }^{1}$ H NMR $\left(300 \mathrm{MHz}, \mathrm{CDCl}_{3}\right): \delta$ 7.76-7.73 $(\mathrm{m}, 2 \mathrm{H}), 7.69-7.66(\mathrm{~m}, 2 \mathrm{H}), 7.46-7.43$ (m, 2H), 7.11-7.09 (m, 3H), 4.46-4.35 (m, 1H), $3.68(\mathrm{dd}, J=10.8,13.2 \mathrm{~Hz}, 1 \mathrm{H})$, $3.17(\mathrm{dd}, J=4.5,13.2 \mathrm{~Hz}, 1 \mathrm{H}), 2.16-2.03(\mathrm{~m}, 1 \mathrm{H}), 1.84-1.74(\mathrm{~m}, 1 \mathrm{H}), 1.25-1.22$ $(\mathrm{m}, 8 \mathrm{H}), 0.82(\mathrm{t}, J=6.6 \mathrm{~Hz}, 3 \mathrm{H}) \mathrm{ppm}$.

${ }^{13}$ C NMR $\left(75 \mathrm{MHz}, \mathrm{CDCl}_{3}\right): \delta 168.6,133.9,133.8,131.9,129.1,127.3,123.3$, 52.9, 32.9, 31.7, 30.4, 28.9, 26.8, 22.7, $14.1 \mathrm{ppm}$.

IR $\left(\mathrm{KBr}, \mathrm{cm}^{-1}\right): 2926,2856,1771,1711,1613,1578,1467,1392,1370,1075,719$, 531.

HRMS (ESI ${ }^{+}$): calcd for $\left[\mathrm{C}_{22} \mathrm{H}_{25} \mathrm{NO}_{2} \mathrm{Se}\right]^{+}:$415.1051, found 415.1053 .

Table 1. The Data Summarized from the HSQC Spectrum of $\mathbf{3 r}$

\begin{tabular}{cccc}
\hline Entry & $\delta_{\mathrm{C}} / \mathrm{ppm}$ & Order of $\mathrm{C}$ atoms & Directly connected $\mathrm{H}, \delta_{\mathrm{H}} / \mathrm{ppm}$ \\
\hline 1 & 168.6 & $\mathrm{C}$ & $7.68,7.45$ \\
2 & $133.9,133.8$ & $2 \mathrm{CH}, 2 \mathrm{CH}$ & \\
3 & 131.9 & $\mathrm{C}$ & 7.10 \\
5 & 129.1 & $2 \mathrm{CH}$ & 7.75 \\
6 & 127.3 & $\mathrm{CH}$ & 4.41 \\
7 & 123.3 & $2 \mathrm{CH}$ & $2.10,1.79$ \\
8 & 52.9 & $\mathrm{CH}$ & $3.68,3.17$ \\
9 & 32.9 & $2 \mathrm{CH}$ & \\
10 & 30.4 & $2 \mathrm{CH}$ & 1.24 \\
11 & 31.7 & $\mathrm{CH}_{2}$ & \\
12 & 28.9 & $\mathrm{CH}_{2}$ & 0.82 \\
13 & 26.8 & $\mathrm{CH}_{2}$ & \\
14 & 22.7 & $\mathrm{CH}_{2}$ & $\mathrm{CH}_{3}$ \\
15 & 14.1 & & \\
\hline
\end{tabular}

Table 2. The Data Summarized from the HMBC Spectrum of $\mathbf{3 r}$

\begin{tabular}{cccl}
\hline entry & $\delta_{\mathrm{C}} / \mathrm{ppm}$ & $\begin{array}{r}\text { H atoms with a long-range } \\
\text { coupling, } \delta_{\mathrm{H}} / \mathrm{ppm}\end{array}$ & $\begin{array}{l}\text { H atoms with the }{ }^{1} J \\
\text { coupling, } \delta_{\mathrm{H}} / \mathrm{ppm}\end{array}$ \\
\hline 1 & 168.6 & $7.75,4.41,(7.68)$ & \\
\hline
\end{tabular}




\begin{tabular}{cccc}
\hline 2 & $133.9,133.8$ & $7.75,7.10$ & $7.68,7.45$ \\
3 & 131.9 & $7.75,7.68$ & 7.10 \\
5 & 129.1 & $7.45,7.10,3.68,3.17$ & \\
6 & 127.3 & 7.45 & \\
7 & 123.3 & 7.68 & \\
8 & 52.9 & 3.68 & \\
9 & 32.9 & $4.41,3.68,3.17$ & 1.24 \\
10 & 30.4 & $4.41,(1.24)$ & \\
11 & 31.7 & $4.41,1.24,0.82$ & \\
12 & 28.9 & $2.10,1.79$ & \\
13 & 26.8 & $2.10,1.79$ & \\
14 & 22.7 & $1.24,0.82$ & \\
15 & 14.1 & 1.24 & \\
\hline
\end{tabular}

\section{2-(2-Phenylseleno-1-octyl)-1H-isoindole-1,3(2H)-dione (3r')}

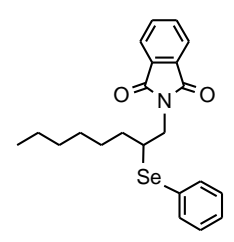

Colorless viscous liquid.

Yield: $21 \mathrm{mg}, 10 \%$.

${ }^{1}$ H NMR $\left(300 \mathrm{MHz}, \mathrm{CDCl}_{3}\right): \delta$ 7.78-7.75 $(\mathrm{m}, 2 \mathrm{H}), 7.69-7.66(\mathrm{~m}, 2 \mathrm{H})$, 7.54-7.50 $(\mathrm{m}, 2 \mathrm{H}), 7.13-7.11(\mathrm{~m}, 3 \mathrm{H}), 4.02-3.86(\mathrm{~m}, 2 \mathrm{H}), 3.74-3.66(\mathrm{~m}, 1 \mathrm{H}), 1.69-1.41(\mathrm{~m}$, $2 \mathrm{H}), 1.26(\mathrm{~m}, 8 \mathrm{H}), 0.89-0.84(\mathrm{~m}, 3 \mathrm{H}) \mathrm{ppm}$.

${ }^{13}$ C NMR (75 MHz, $\left.\mathrm{CDCl}_{3}\right): \delta 168.4,134.4,134.0,132.0,129.0,127.4,123.4$, 43.9, 43.2, 32.8, 31.8, 29.1, 27.7, 22.7, $14.2 \mathrm{ppm}$.

IR $\left(\mathrm{KBr}, \mathrm{cm}^{-1}\right):$ 2927, 2855, 1772, 1715, 1578, 1434, 1394,1071, 721, 693, 530.

HRMS $\left(\mathrm{ESI}^{+}\right)$: calcd for $\left[\mathrm{C}_{22} \mathrm{H}_{25} \mathrm{NO}_{2} \mathrm{Se}\right]^{+}: 415.1051$, found 415.1056 .

2-[1-Phenyl-2-(phenylseleno)propyl]-1H-isoindole-1,3(2H)-dione $( \pm 3 \mathrm{~s})$

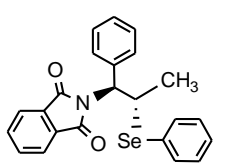

Yellow liquid.

Yield: $111 \mathrm{mg}, 53 \%$.

${ }^{1}$ H NMR (400 MHz, $\left.\mathrm{CDCl}_{3}\right): \delta$ 7.76-7.74 (m, 2H), 7.67-7.65 (m, 2H), $7.59(\mathrm{~d}, J=$ $7.2 \mathrm{~Hz}, 2 \mathrm{H}), 7.54-7.52(\mathrm{~m}, 2 \mathrm{H}), 7.33-7.25(\mathrm{~m}, 3 \mathrm{H}), 7.20-7.15(\mathrm{~m}, 3 \mathrm{H}), 5.22(\mathrm{~d}, J=$ $11.6 \mathrm{~Hz}, 1 \mathrm{H}), 4.88-4.79(\mathrm{~m}, 1 \mathrm{H}), 1.28(\mathrm{~d}, J=6.8 \mathrm{~Hz}, 3 \mathrm{H}) \mathrm{ppm}$.

${ }^{13}$ C NMR $\left(100 \mathrm{MHz}, \mathrm{CDCl}_{3}\right): \delta 168.1,138.2,136.3,133.8,131.8,128.9,128.8$, 128.7, 128.2, 128.2, 128.0, 123.2, 61.1. 37.9, 20.6 ppm.

IR $\left(\mathrm{KBr}, \mathrm{cm}^{-1}\right): 3059,2915,1765,1709,1615,1445,1379,1180,1012,714$.

HRMS $\left(\mathrm{ESI}^{+}\right)$: calcd for $\left[\mathrm{C}_{23} \mathrm{H}_{19} \mathrm{NO}_{2} \mathrm{NaSe}\right]^{+}: 444.0479$, found 444.0469 .

\section{Characterization of products $4 a, 9 a, 5 a, 5 a^{\prime}, 5 s$, and $5 s^{\prime}$}

\section{1-[1-Phenyl- 2-(phenylseleno)ethyl]-2,5-pyrrolidinedione (4a)}

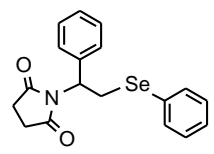

Brown viscous liquid.

Yield: $90 \mathrm{mg}, 50 \%$.

${ }^{1} \mathbf{H}$ NMR $\left(300 \mathrm{MHz}, \mathrm{CDCl}_{3}\right): \delta$ 7.55-7.52 (m, 2H), 7.44-7.41 (m, 2H), 7.30-7.24 (m, $6 \mathrm{H}), 5.38(\mathrm{dd}, J=4.5,11.4 \mathrm{~Hz}, 1 \mathrm{H}), 4.24(\mathrm{dd}, J=11.4,12.6 \mathrm{~Hz}, 1 \mathrm{H}), 3.39(\mathrm{dd}, J=$ $4.5,12.6 \mathrm{~Hz}, 1 \mathrm{H}), 2.51-2.37(\mathrm{~m}, 4 \mathrm{H}) \mathrm{ppm}$. 
${ }^{13} \mathbf{C}$ NMR (75 MHz, $\left.\mathrm{CDCl}_{3}\right): \delta 177.1,138.6,134.1,129.2,128.6,128.3,128.1,127.8$, 56.3, 28.0, $27.9 \mathrm{ppm}$.

IR $\left(\mathrm{KBr}, \mathrm{cm}^{-1}\right): 2030,1769,1700,1365,1183,1137,741,693$.

HRMS (ESI ${ }^{+}$): calcd for $\left[\mathrm{C}_{18} \mathrm{H}_{17} \mathrm{KNO}_{2} \mathrm{Se}\right]^{+}: 398.0062$, found 398.0056 .

1-Phenyl- 2-(phenylseleno)ethanol (9a) ${ }^{[5]}$

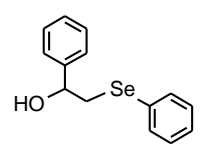

Colorless viscous liquid.

Yield: $69 \mathrm{mg}, 50 \%$.

${ }^{1} \mathbf{H}$ NMR $\left(300 \mathrm{MHz}, \mathrm{CDCl}_{3}\right): \delta$ 7.50-7.47 (m, 2H), 7.29-7.21 (m, 8H), 4.71-4.67 (m, $1 \mathrm{H}), 3.25-3.20(\mathrm{~m}, 1 \mathrm{H}), 3.12-3.05(\mathrm{~m}, 1 \mathrm{H}), 3.03(\mathrm{~s}, 1 \mathrm{H})$.

${ }^{13}$ C NMR (300MHz, $\mathrm{CDCl}_{3}$ ): $\delta$ 142.7, 133.1, 129.5, 129.3, 128.6, 128.0, 127.4, 125.9, 72.4, 38.3.

2-[1-(3'-methoxy-4'-ethoxy)phenyl-1-propenyl]-1H-isoindole-1,3(2H)-dione (5a)

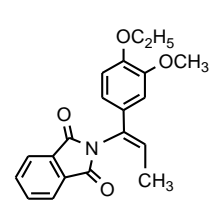

Light yellow solid.

Mp: $145.6-146.0^{\circ} \mathrm{C}$.

Yield: $54 \mathrm{mg}, 80 \%$.

${ }^{1}$ H NMR $\left(300 \mathrm{MHz}, \mathrm{CDCl}_{3}\right): \delta$ 7.96-7.92 (m, 2H), 7.82-7.78 (m, 2H), $6.96(\mathrm{~s}, 1 \mathrm{H})$, $6.76(\mathrm{~d}, J=0.9 \mathrm{~Hz}, 2 \mathrm{H}), 6.41(\mathrm{q}, \mathrm{J}=6.9 \mathrm{~Hz}, 1 \mathrm{H}), 4.07(\mathrm{q}, 2 \mathrm{H}), 3.85(\mathrm{~d}, J=4.5 \mathrm{~Hz}$, $3 \mathrm{H}), 1.74(\mathrm{~d}, J=6.9 \mathrm{~Hz}, 3 \mathrm{H}), 1.44(\mathrm{t}, J=6.9 \mathrm{~Hz}, 3 \mathrm{H}) \mathrm{ppm}$.

${ }^{13}$ C NMR $\left(75 \mathrm{MHz}, \mathrm{CDCl}_{3}\right): \delta 167.3,149.5,148.8,134.5,132.2,130.7,129.5,125.8$, $123.9,117.9,112.6,109.0,64.5,56.2,14.9,14.2 \mathrm{ppm}$.

IR $\left(\mathrm{KBr}, \mathrm{cm}^{-1}\right): 2974,2917,1770,1721,1598,1599,1466,1373,1105,1041$, 883,723 .

HRMS (ESI ${ }^{+}$): calcd for $\left[\mathrm{C}_{20} \mathrm{H}_{19} \mathrm{NO}_{4}\right]^{+}: 337.1314$, found 337.1318.

2-[3-(3'-methoxy-4'-ethoxy)phenyl-3-propenyl]-1H-isoindole-1,3(2H)-dione (5a')

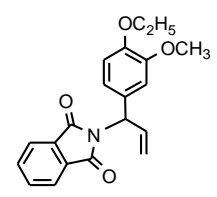

Yellow viscous liquid.

Yield: $13 \mathrm{mg}, 20 \%$.

${ }^{1} \mathbf{H}$ NMR $\left(300 \mathrm{MHz}, \mathrm{CDCl}_{3}\right): \delta$ 7.84-7.80 (m, 2H), 7.73-7.69 (m, 2H), 7.05-6.99 (m, $2 \mathrm{H}), 6.81(\mathrm{~d}, J=8.4 \mathrm{~Hz}, 1 \mathrm{H}), 6.69-6.58(\mathrm{~m}, 1 \mathrm{H}), 5.90(\mathrm{~d}, J=7.5 \mathrm{~Hz}, 1 \mathrm{H}), 5.36-5.29$ $(\mathrm{m}, 2 \mathrm{H}), 4.07(\mathrm{q}, 2 \mathrm{H}), 3.86(\mathrm{~s}, 3 \mathrm{H}), 1.44(\mathrm{t}, J=6.9 \mathrm{~Hz}, 3 \mathrm{H}) \mathrm{ppm}$.

${ }^{13} \mathbf{C}$ NMR $\left(75 \mathrm{MHz}, \mathrm{CDCl}_{3}\right): \delta 167.9,149.4,148.2,134.62,134.1,132.1,131.2,123.5$, $120.5,118.7,112.6,111.9,64.4,56.9,56.1,14.9 \mathrm{ppm}$.

IR $\left(\mathrm{KBr}, \mathrm{cm}^{-1}\right): 2925,1769,1712,1602,1514,1462,1380,1262,1140,1084,1038$ 721.

HRMS $\left(\mathrm{ESI}^{+}\right)$: calcd for $\left[\mathrm{C}_{20} \mathrm{H}_{19} \mathrm{NO}_{4} \mathrm{Na}\right]^{+}: 360.1212$, found 360.1204 .

(Z)-2-[1-phenyl-1-propenyl]-1H-isoindole-1,3(2H)-dione (5c)

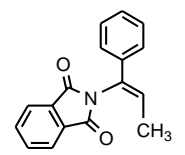

Light yellow solid

Mp: $137.6-138.2^{\circ} \mathrm{C}$.

Yield: $49 \mathrm{mg}, 93 \%$.

${ }^{1} \mathbf{H}$ NMR $\left(300 \mathrm{MHz}, \mathrm{CDCl}_{3}\right): \delta$ 7.96-7.92 (m, 2H), 7.82-7.78 (m, 2H), 7.35-7.25 (m, $5 \mathrm{H}), 6.52(\mathrm{q}, J=7.2,14.1 \mathrm{~Hz}, 1 \mathrm{H}), 1.77(\mathrm{~d}, J=6.9 \mathrm{~Hz}, 3 \mathrm{H}) \mathrm{ppm}$.

${ }^{13} \mathbf{C}$ NMR $\left(75 \mathrm{MHz}, \mathrm{CDCl}_{3}\right): \delta 167.2,136.5,134.5,132.2,130.9,128.8,128.3,127.5$, 125.1, 124.0, $14.3 \mathrm{ppm}$.

IR $\left(\mathrm{KBr}, \mathrm{cm}^{-1}\right): 3036,2920,2848,1773,1719,1455,1379,1290,1115,1084,723$.

HRMS (ESI ${ }^{+}$): calcd for $\left[\mathrm{C}_{17} \mathrm{H}_{13} \mathrm{NO}_{2} \mathrm{Na}\right]^{+}: 286.0844$, found 286.0836 . 
(E)-2-[1-phenyl-1-propenyl]-1H-isoindole-1,3(2H)-dione $(5 \mathrm{~s})$

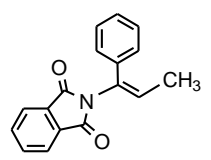

White solid

Mp: $103.6-104.5^{\circ} \mathrm{C}$.

Yield: $47 \mathrm{mg}, 90 \%$.

${ }^{1}$ H NMR $\left(400 \mathrm{MHz}, \mathrm{CDCl}_{3}\right): \delta$ 7.88-7.85 (m, 2H), 7.74-7.72 (m, 2H), 7.37-7.26 (m, $5 \mathrm{H}), 5.98$ (q, $J=7.2,14.4 \mathrm{~Hz}, 1 \mathrm{H}), 1.95(\mathrm{~d}, J=6.9 \mathrm{~Hz}, 3 \mathrm{H}) \mathrm{ppm}$.

${ }^{13}$ C NMR $\left(100 \mathrm{MHz}, \mathrm{CDCl}_{3}\right): \delta 167.7,135.4,134.1,132.0,130.0,129.4,128.6,128.3$, 128.1, 123.6, $14.4 \mathrm{ppm}$.

IR $\left(\mathrm{KBr}, \mathrm{cm}^{-1}\right): 2913,1773,1717,1615,1454,1387,1171,1084,712$.

HRMS $\left(\mathrm{ESI}^{+}\right)$: calcd for $\left[\mathrm{C}_{17} \mathrm{H}_{13} \mathrm{NO}_{2} \mathrm{Na}\right]^{+}: 286.0844$, found 286.0837 .

\section{X-ray structure of compound 3o}
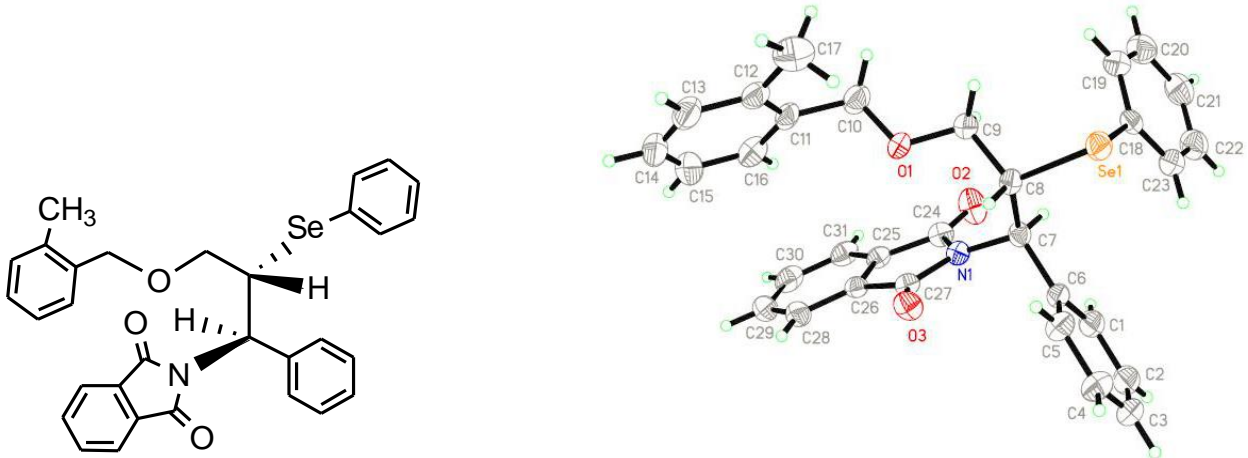

Table 1. Crystal data and structure refinement for $\mathbf{3 o}$.

\begin{tabular}{ll}
\hline Identification code & $\mathbf{3 o}$ \\
Empirical formula & $\mathrm{C} 31 \mathrm{H} 27 \mathrm{~N} \mathrm{O} 3 \mathrm{Se}$ \\
Formula weight & 540.50 \\
Temperature & $293(2) \mathrm{K}$ \\
Wavelength & $0.71073 \AA$ \\
Crystal system & Triclinic \\
Space group & $\mathrm{P}-1$ \\
Unit cell dimensions & $\mathrm{a}=6.8204(8) \AA \quad \quad \alpha=72.8040(10)^{\circ}$. \\
& $\mathrm{b}=13.7059(15) \AA \quad \beta=87.4050(10)^{\circ}$. \\
& $\mathrm{c}=14.4554(16) \AA \quad \gamma=88.7930(10)^{\circ}$. \\
Volume & $1289.5(3) \AA^{3}$ \\
Z & 2 \\
Calculated density & $1.392 \mathrm{Mg} / \mathrm{m}^{3}$ \\
Absorption coefficient & $1.489 \mathrm{~mm}^{-1}$ \\
F(000) & 556 \\
Crystal size & $0.34 \times 0.30 \times 0.28 \mathrm{~mm}{ }^{3}$ \\
Theta range for data collection & 1.48 to $25.15^{\circ}$. \\
Limiting indices & $-8<=\mathrm{h}<=8,-16<=\mathrm{k}<=16,-17<=1<=17$ \\
Reflections collected / unique & $10298 / 4592[\mathrm{R}(\mathrm{int})=0.0364]$ \\
Completeness to theta $=25.15$ & $99.5 \%$ \\
Max. and min. transmission & $0.6806 \mathrm{and} 0.6315^{\circ}$ \\
\hline
\end{tabular}




\begin{tabular}{ll}
\hline Refinement method & Full-matrix least-squares on $\mathrm{F}^{2}$ \\
Data / restraints / parameters & $4592 / 0 / 326$ \\
Goodness-of-fit on $\mathrm{F}^{2}$ & 1.047 \\
Final R indices [I>2sigma(I)] & $\mathrm{R} 1=0.0398, \mathrm{wR} 2=0.0776$ \\
R indices (all data) & $\mathrm{R} 1=0.0645, \mathrm{wR} 2=0.0868$ \\
Largest diff. peak and hole & 0.314 and -0.285 e. $\mathrm{A}^{-3}$ \\
\hline
\end{tabular}

Table 2. Atomic coordinates ( $\times 10^{4}$ ) and equivalent isotropic displacement parameters $\left(\mathrm{A}^{2} \times 10^{3}\right)$ for 3 o.

\begin{tabular}{|c|c|c|c|c|}
\hline & $\mathrm{x}$ & $\mathrm{y}$ & $\mathrm{z}$ & $\mathrm{U}(\mathrm{eq})^{a}$ \\
\hline $\mathrm{N}(1)$ & $3008(3)$ & $2850(2)$ & $1597(2)$ & $41(1)$ \\
\hline $\mathrm{O}(1)$ & $698(3)$ & $3125(2)$ & 3197(1) & $54(1)$ \\
\hline $\mathrm{O}(2)$ & $5920(3)$ & $2377(2)$ & $2366(2)$ & $74(1)$ \\
\hline $\mathrm{O}(3)$ & $389(3)$ & $3810(2)$ & $849(2)$ & $55(1)$ \\
\hline $\operatorname{Se}(1)$ & $-466(1)$ & $275(1)$ & $3003(1)$ & $54(1)$ \\
\hline $\mathrm{C}(1)$ & $3293(5)$ & $1202(2)$ & $436(2)$ & $60(1)$ \\
\hline $\mathrm{C}(2)$ & $3039(7)$ & 994(3) & $-431(3)$ & $77(1)$ \\
\hline$C(3)$ & $1239(8)$ & 1154(3) & $-838(3)$ & $82(1)$ \\
\hline$C(4)$ & $-296(6)$ & $1522(3)$ & $-396(3)$ & $75(1)$ \\
\hline$C(5)$ & $-47(5)$ & 1734(3) & $469(2)$ & $59(1)$ \\
\hline$C(6)$ & $1744(5)$ & $1578(2)$ & $894(2)$ & $45(1)$ \\
\hline$C(7)$ & $2170(4)$ & $1820(2)$ & $1829(2)$ & $43(1)$ \\
\hline $\mathrm{C}(8)$ & $470(4)$ & 1692(2) & $2578(2)$ & $43(1)$ \\
\hline $\mathrm{C}(9)$ & $926(5)$ & $2050(2)$ & $3444(2)$ & $50(1)$ \\
\hline$C(10)$ & $1233(5)$ & $3526(3)$ & $3942(2)$ & $64(1)$ \\
\hline $\mathrm{C}(11)$ & $546(5)$ & $4616(3)$ & $3705(2)$ & $54(1)$ \\
\hline $\mathrm{C}(12)$ & $-1391(6)$ & 4871(3) & $3924(2)$ & $59(1)$ \\
\hline$C(13)$ & $-1890(6)$ & 5893(3) & $3695(3)$ & $75(1)$ \\
\hline $\mathrm{C}(14)$ & $-575(8)$ & $6646(3)$ & $3255(3)$ & $86(1)$ \\
\hline$C(15)$ & $1281(8)$ & $6397(3)$ & $3030(3)$ & $86(1)$ \\
\hline$C(16)$ & $1840(6)$ & 5391(3) & $3259(3)$ & $70(1)$ \\
\hline$C(17)$ & $-2876(6)$ & $4064(4)$ & $4385(3)$ & $91(1)$ \\
\hline $\mathrm{C}(18)$ & 1934(5) & $-439(2)$ & $3431(2)$ & $50(1)$ \\
\hline C(19) & $2543(6)$ & $-579(3)$ & $4357(2)$ & $64(1)$ \\
\hline $\mathrm{C}(20)$ & $4216(7)$ & $-1140(3)$ & $4672(3)$ & $82(1)$ \\
\hline $\mathrm{C}(21)$ & $5311(6)$ & $-1566(3)$ & $4065(3)$ & $80(1)$ \\
\hline $\mathrm{C}(22)$ & $4716(6)$ & $-1432(3)$ & $3148(3)$ & $76(1)$ \\
\hline $\mathrm{C}(23)$ & $3040(6)$ & $-880(3)$ & $2827(3)$ & $63(1)$ \\
\hline $\mathrm{C}(24)$ & 4832(4) & $3035(3)$ & $1917(2)$ & $48(1)$ \\
\hline$C(25)$ & $5066(4)$ & $4161(2)$ & $1603(2)$ & $44(1)$ \\
\hline$C(26)$ & $3387(4)$ & $4603(2)$ & $1146(2)$ & $42(1)$ \\
\hline $\mathrm{C}(27)$ & 2018(5) & $3763(2)$ & $1153(2)$ & $43(1)$ \\
\hline$C(28)$ & $3157(5)$ & $5634(3)$ & $770(2)$ & $55(1)$ \\
\hline $\mathrm{C}(29)$ & $4710(6)$ & $6234(3)$ & $867(3)$ & $66(1)$ \\
\hline
\end{tabular}




\begin{tabular}{lllll}
$\mathrm{C}(30)$ & $6401(6)$ & $5799(3)$ & $1321(3)$ & $68(1)$ \\
$\mathrm{C}(31)$ & $6619(5)$ & $4755(3)$ & $1701(2)$ & $57(1)$ \\
\hline
\end{tabular}

${ }^{a} \mathrm{U}(\mathrm{eq})$ is defined as one third of the trace of the orthogonalized $\mathrm{U}^{\mathrm{ij}}$ tensor.

Table 3. Bond lengths $[\AA ̊]$ and angles $\left[^{\circ}\right]$ for 30 .

\begin{tabular}{|c|c|}
\hline $\mathrm{N}(1)-\mathrm{C}(24)$ & $1.400(4)$ \\
\hline $\mathrm{N}(1)-\mathrm{C}(27)$ & $1.402(4)$ \\
\hline $\mathrm{N}(1)-\mathrm{C}(7)$ & $1.472(4)$ \\
\hline $\mathrm{O}(1)-\mathrm{C}(10)$ & $1.411(3)$ \\
\hline $\mathrm{O}(1)-\mathrm{C}(9)$ & $1.416(3)$ \\
\hline $\mathrm{O}(2)-\mathrm{C}(24)$ & $1.207(3)$ \\
\hline $\mathrm{O}(3)-\mathrm{C}(27)$ & $1.207(3)$ \\
\hline $\operatorname{Se}(1)-C(18)$ & $1.920(3)$ \\
\hline $\operatorname{Se}(1)-C(8)$ & $1.967(3)$ \\
\hline $\mathrm{C}(1)-\mathrm{C}(2)$ & $1.384(5)$ \\
\hline $\mathrm{C}(1)-\mathrm{C}(6)$ & $1.392(4)$ \\
\hline $\mathrm{C}(1)-\mathrm{H}(1)$ & 0.9300 \\
\hline$C(2)-C(3)$ & $1.371(5)$ \\
\hline $\mathrm{C}(2)-\mathrm{H}(2)$ & 0.9300 \\
\hline $\mathrm{C}(3)-\mathrm{C}(4)$ & $1.366(5)$ \\
\hline $\mathrm{C}(3)-\mathrm{H}(3)$ & 0.9300 \\
\hline$C(4)-C(5)$ & $1.383(4)$ \\
\hline $\mathrm{C}(4)-\mathrm{H}(4)$ & 0.9300 \\
\hline$C(5)-C(6)$ & $1.376(4)$ \\
\hline $\mathrm{C}(5)-\mathrm{H}(5)$ & 0.9300 \\
\hline$C(6)-C(7)$ & $1.525(4)$ \\
\hline$C(7)-C(8)$ & $1.527(4)$ \\
\hline $\mathrm{C}(7)-\mathrm{H}(7)$ & 0.9800 \\
\hline $\mathrm{C}(8)-\mathrm{C}(9)$ & $1.520(4)$ \\
\hline $\mathrm{C}(8)-\mathrm{H}(8)$ & 0.9800 \\
\hline $\mathrm{C}(9)-\mathrm{H}(9 \mathrm{~A})$ & 0.9700 \\
\hline $\mathrm{C}(9)-\mathrm{H}(9 \mathrm{~B})$ & 0.9700 \\
\hline $\mathrm{C}(10)-\mathrm{C}(11)$ & $1.501(4)$ \\
\hline $\mathrm{C}(10)-\mathrm{H}(10 \mathrm{~A})$ & 0.9700 \\
\hline $\mathrm{C}(10)-\mathrm{H}(10 \mathrm{~B})$ & 0.9700 \\
\hline $\mathrm{C}(11)-\mathrm{C}(16)$ & $1.380(5)$ \\
\hline $\mathrm{C}(11)-\mathrm{C}(12)$ & $1.406(5)$ \\
\hline $\mathrm{C}(12)-\mathrm{C}(13)$ & $1.379(5)$ \\
\hline $\mathrm{C}(12)-\mathrm{C}(17)$ & $1.499(5)$ \\
\hline $\mathrm{C}(13)-\mathrm{C}(14)$ & $1.369(6)$ \\
\hline $\mathrm{C}(13)-\mathrm{H}(13)$ & 0.9300 \\
\hline$C(14)-C(15)$ & $1.354(6)$ \\
\hline
\end{tabular}




\begin{tabular}{|c|c|}
\hline $\mathrm{C}(14)-\mathrm{H}(14)$ & 0.9300 \\
\hline $\mathrm{C}(15)-\mathrm{C}(16)$ & $1.370(5)$ \\
\hline $\mathrm{C}(15)-\mathrm{H}(15)$ & 0.9300 \\
\hline $\mathrm{C}(16)-\mathrm{H}(16)$ & 0.9300 \\
\hline $\mathrm{C}(17)-\mathrm{H}(17 \mathrm{~A})$ & 0.9600 \\
\hline C(17)-H(17B) & 0.9600 \\
\hline $\mathrm{C}(17)-\mathrm{H}(17 \mathrm{C})$ & 0.9600 \\
\hline C(18)-C(19) & $1.377(4)$ \\
\hline $\mathrm{C}(18)-\mathrm{C}(23)$ & $1.390(4)$ \\
\hline $\mathrm{C}(19)-\mathrm{C}(20)$ & $1.380(5)$ \\
\hline C(19)-H(19) & 0.9300 \\
\hline $\mathrm{C}(20)-\mathrm{C}(21)$ & $1.376(5)$ \\
\hline $\mathrm{C}(20)-\mathrm{H}(20)$ & 0.9300 \\
\hline $\mathrm{C}(21)-\mathrm{C}(22)$ & $1.363(5)$ \\
\hline $\mathrm{C}(21)-\mathrm{H}(21)$ & 0.9300 \\
\hline $\mathrm{C}(22)-\mathrm{C}(23)$ & $1.378(5)$ \\
\hline $\mathrm{C}(22)-\mathrm{H}(22)$ & 0.9300 \\
\hline $\mathrm{C}(23)-\mathrm{H}(23)$ & 0.9300 \\
\hline $\mathrm{C}(24)-\mathrm{C}(25)$ & $1.484(4)$ \\
\hline $\mathrm{C}(25)-\mathrm{C}(26)$ & $1.384(4)$ \\
\hline$C(25)-C(31)$ & $1.387(4)$ \\
\hline$C(26)-C(28)$ & $1.365(4)$ \\
\hline $\mathrm{C}(26)-\mathrm{C}(27)$ & $1.494(4)$ \\
\hline $\mathrm{C}(28)-\mathrm{C}(29)$ & $1.391(5)$ \\
\hline $\mathrm{C}(28)-\mathrm{H}(28)$ & 0.9300 \\
\hline $\mathrm{C}(29)-\mathrm{C}(30)$ & $1.387(5)$ \\
\hline C(29)-H(29) & 0.9300 \\
\hline $\mathrm{C}(30)-\mathrm{C}(31)$ & $1.381(5)$ \\
\hline $\mathrm{C}(30)-\mathrm{H}(30)$ & 0.9300 \\
\hline $\mathrm{C}(31)-\mathrm{H}(31)$ & 0.9300 \\
\hline $\mathrm{C}(24)-\mathrm{N}(1)-\mathrm{C}(27)$ & $111.4(3)$ \\
\hline $\mathrm{C}(24)-\mathrm{N}(1)-\mathrm{C}(7)$ & $122.8(3)$ \\
\hline $\mathrm{C}(27)-\mathrm{N}(1)-\mathrm{C}(7)$ & $125.3(2)$ \\
\hline $\mathrm{C}(10)-\mathrm{O}(1)-\mathrm{C}(9)$ & $112.8(2)$ \\
\hline $\mathrm{C}(18)-\mathrm{Se}(1)-\mathrm{C}(8)$ & $100.32(12)$ \\
\hline $\mathrm{C}(2)-\mathrm{C}(1)-\mathrm{C}(6)$ & $120.6(4)$ \\
\hline $\mathrm{C}(2)-\mathrm{C}(1)-\mathrm{H}(1)$ & 119.7 \\
\hline $\mathrm{C}(6)-\mathrm{C}(1)-\mathrm{H}(1)$ & 119.7 \\
\hline $\mathrm{C}(3)-\mathrm{C}(2)-\mathrm{C}(1)$ & $119.5(4)$ \\
\hline $\mathrm{C}(3)-\mathrm{C}(2)-\mathrm{H}(2)$ & 120.2 \\
\hline $\mathrm{C}(1)-\mathrm{C}(2)-\mathrm{H}(2)$ & 120.2 \\
\hline $\mathrm{C}(4)-\mathrm{C}(3)-\mathrm{C}(2)$ & $120.5(4)$ \\
\hline $\mathrm{C}(4)-\mathrm{C}(3)-\mathrm{H}(3)$ & 119.8 \\
\hline
\end{tabular}




\begin{tabular}{|c|c|}
\hline $\mathrm{C}(2)-\mathrm{C}(3)-\mathrm{H}(3)$ & 119.8 \\
\hline$C(3)-C(4)-C(5)$ & $120.2(4)$ \\
\hline $\mathrm{C}(3)-\mathrm{C}(4)-\mathrm{H}(4)$ & 119.9 \\
\hline $\mathrm{C}(5)-\mathrm{C}(4)-\mathrm{H}(4)$ & 119.9 \\
\hline$C(6)-C(5)-C(4)$ & 120.6(3) \\
\hline $\mathrm{C}(6)-\mathrm{C}(5)-\mathrm{H}(5)$ & 119.7 \\
\hline $\mathrm{C}(4)-\mathrm{C}(5)-\mathrm{H}(5)$ & 119.7 \\
\hline $\mathrm{C}(5)-\mathrm{C}(6)-\mathrm{C}(1)$ & 118.6(3) \\
\hline$C(5)-C(6)-C(7)$ & 124.2(3) \\
\hline $\mathrm{C}(1)-\mathrm{C}(6)-\mathrm{C}(7)$ & $117.2(3)$ \\
\hline $\mathrm{N}(1)-\mathrm{C}(7)-\mathrm{C}(6)$ & $109.7(2)$ \\
\hline $\mathrm{N}(1)-\mathrm{C}(7)-\mathrm{C}(8)$ & $110.7(2)$ \\
\hline $\mathrm{C}(6)-\mathrm{C}(7)-\mathrm{C}(8)$ & $116.5(2)$ \\
\hline $\mathrm{N}(1)-\mathrm{C}(7)-\mathrm{H}(7)$ & 106.5 \\
\hline $\mathrm{C}(6)-\mathrm{C}(7)-\mathrm{H}(7)$ & 106.5 \\
\hline $\mathrm{C}(8)-\mathrm{C}(7)-\mathrm{H}(7)$ & 106.5 \\
\hline $\mathrm{C}(9)-\mathrm{C}(8)-\mathrm{C}(7)$ & $113.8(2)$ \\
\hline $\mathrm{C}(9)-\mathrm{C}(8)-\mathrm{Se}(1)$ & 110.7(2) \\
\hline $\mathrm{C}(7)-\mathrm{C}(8)-\mathrm{Se}(1)$ & $111.08(19$ \\
\hline $\mathrm{C}(9)-\mathrm{C}(8)-\mathrm{H}(8)$ & 106.9 \\
\hline $\mathrm{C}(7)-\mathrm{C}(8)-\mathrm{H}(8)$ & 106.9 \\
\hline $\mathrm{Se}(1)-\mathrm{C}(8)-\mathrm{H}(8)$ & 106.9 \\
\hline $\mathrm{O}(1)-\mathrm{C}(9)-\mathrm{C}(8)$ & $109.4(2)$ \\
\hline $\mathrm{O}(1)-\mathrm{C}(9)-\mathrm{H}(9 \mathrm{~A})$ & 109.8 \\
\hline $\mathrm{C}(8)-\mathrm{C}(9)-\mathrm{H}(9 \mathrm{~A})$ & 109.8 \\
\hline $\mathrm{O}(1)-\mathrm{C}(9)-\mathrm{H}(9 \mathrm{~B})$ & 109.8 \\
\hline $\mathrm{C}(8)-\mathrm{C}(9)-\mathrm{H}(9 \mathrm{~B})$ & 109.8 \\
\hline $\mathrm{H}(9 \mathrm{~A})-\mathrm{C}(9)-\mathrm{H}(9 \mathrm{~B})$ & 108.2 \\
\hline $\mathrm{O}(1)-\mathrm{C}(10)-\mathrm{C}(11)$ & $108.7(3)$ \\
\hline $\mathrm{O}(1)-\mathrm{C}(10)-\mathrm{H}(10 \mathrm{~A})$ & 110.0 \\
\hline $\mathrm{C}(11)-\mathrm{C}(10)-\mathrm{H}(10 \mathrm{~A})$ & 110.0 \\
\hline $\mathrm{O}(1)-\mathrm{C}(10)-\mathrm{H}(10 \mathrm{~B})$ & 110.0 \\
\hline $\mathrm{C}(11)-\mathrm{C}(10)-\mathrm{H}(10 \mathrm{~B})$ & 110.0 \\
\hline $\mathrm{H}(10 \mathrm{~A})-\mathrm{C}(10)-\mathrm{H}(10 \mathrm{~B})$ & 108.3 \\
\hline $\mathrm{C}(16)-\mathrm{C}(11)-\mathrm{C}(12)$ & $118.9(3)$ \\
\hline $\mathrm{C}(16)-\mathrm{C}(11)-\mathrm{C}(10)$ & 119.4(3) \\
\hline $\mathrm{C}(12)-\mathrm{C}(11)-\mathrm{C}(10)$ & $121.6(3)$ \\
\hline $\mathrm{C}(13)-\mathrm{C}(12)-\mathrm{C}(11)$ & $117.8(4)$ \\
\hline $\mathrm{C}(13)-\mathrm{C}(12)-\mathrm{C}(17)$ & $120.8(4)$ \\
\hline $\mathrm{C}(11)-\mathrm{C}(12)-\mathrm{C}(17)$ & $121.4(3)$ \\
\hline $\mathrm{C}(14)-\mathrm{C}(13)-\mathrm{C}(12)$ & $122.0(4)$ \\
\hline $\mathrm{C}(14)-\mathrm{C}(13)-\mathrm{H}(13)$ & 119.0 \\
\hline $\mathrm{C}(12)-\mathrm{C}(13)-\mathrm{H}(13)$ & 119.0 \\
\hline $\mathrm{C}(15)-\mathrm{C}(14)-\mathrm{C}(13)$ & $120.0(4)$ \\
\hline
\end{tabular}




\begin{tabular}{|c|c|}
\hline $\mathrm{C}(15)-\mathrm{C}(14)-\mathrm{H}(14)$ & 120.0 \\
\hline $\mathrm{C}(13)-\mathrm{C}(14)-\mathrm{H}(14)$ & 120.0 \\
\hline$C(14)-C(15)-C(16)$ & $119.7(4)$ \\
\hline $\mathrm{C}(14)-\mathrm{C}(15)-\mathrm{H}(15)$ & 120.2 \\
\hline $\mathrm{C}(16)-\mathrm{C}(15)-\mathrm{H}(15)$ & 120.2 \\
\hline $\mathrm{C}(15)-\mathrm{C}(16)-\mathrm{C}(11)$ & $121.5(4)$ \\
\hline $\mathrm{C}(15)-\mathrm{C}(16)-\mathrm{H}(16)$ & 119.2 \\
\hline $\mathrm{C}(11)-\mathrm{C}(16)-\mathrm{H}(16)$ & 119.2 \\
\hline $\mathrm{C}(12)-\mathrm{C}(17)-\mathrm{H}(17 \mathrm{~A})$ & 109.5 \\
\hline $\mathrm{C}(12)-\mathrm{C}(17)-\mathrm{H}(17 \mathrm{~B})$ & 109.5 \\
\hline $\mathrm{H}(17 \mathrm{~A})-\mathrm{C}(17)-\mathrm{H}(17 \mathrm{~B})$ & 109.5 \\
\hline $\mathrm{C}(12)-\mathrm{C}(17)-\mathrm{H}(17 \mathrm{C})$ & 109.5 \\
\hline $\mathrm{H}(17 \mathrm{~A})-\mathrm{C}(17)-\mathrm{H}(17 \mathrm{C})$ & 109.5 \\
\hline $\mathrm{H}(17 \mathrm{~B})-\mathrm{C}(17)-\mathrm{H}(17 \mathrm{C})$ & 109.5 \\
\hline $\mathrm{C}(19)-\mathrm{C}(18)-\mathrm{C}(23)$ & $118.3(3)$ \\
\hline $\mathrm{C}(19)-\mathrm{C}(18)-\mathrm{Se}(1)$ & 121.2(3) \\
\hline $\mathrm{C}(23)-\mathrm{C}(18)-\mathrm{Se}(1)$ & $120.3(3)$ \\
\hline $\mathrm{C}(18)-\mathrm{C}(19)-\mathrm{C}(20)$ & $120.6(3)$ \\
\hline C(18)-C(19)-H(19) & 119.7 \\
\hline $\mathrm{C}(20)-\mathrm{C}(19)-\mathrm{H}(19)$ & 119.7 \\
\hline $\mathrm{C}(21)-\mathrm{C}(20)-\mathrm{C}(19)$ & $120.6(4)$ \\
\hline $\mathrm{C}(21)-\mathrm{C}(20)-\mathrm{H}(20)$ & 119.7 \\
\hline $\mathrm{C}(19)-\mathrm{C}(20)-\mathrm{H}(20)$ & 119.7 \\
\hline $\mathrm{C}(22)-\mathrm{C}(21)-\mathrm{C}(20)$ & 119.2(4) \\
\hline $\mathrm{C}(22)-\mathrm{C}(21)-\mathrm{H}(21)$ & 120.4 \\
\hline $\mathrm{C}(20)-\mathrm{C}(21)-\mathrm{H}(21)$ & 120.4 \\
\hline $\mathrm{C}(21)-\mathrm{C}(22)-\mathrm{C}(23)$ & $120.8(4)$ \\
\hline $\mathrm{C}(21)-\mathrm{C}(22)-\mathrm{H}(22)$ & 119.6 \\
\hline $\mathrm{C}(23)-\mathrm{C}(22)-\mathrm{H}(22)$ & 119.6 \\
\hline $\mathrm{C}(22)-\mathrm{C}(23)-\mathrm{C}(18)$ & $120.5(3)$ \\
\hline $\mathrm{C}(22)-\mathrm{C}(23)-\mathrm{H}(23)$ & 119.8 \\
\hline $\mathrm{C}(18)-\mathrm{C}(23)-\mathrm{H}(23)$ & 119.8 \\
\hline $\mathrm{O}(2)-\mathrm{C}(24)-\mathrm{N}(1)$ & $124.3(3)$ \\
\hline $\mathrm{O}(2)-\mathrm{C}(24)-\mathrm{C}(25)$ & $129.6(3)$ \\
\hline $\mathrm{N}(1)-\mathrm{C}(24)-\mathrm{C}(25)$ & 106.1(3) \\
\hline$C(26)-C(25)-C(31)$ & $121.2(3)$ \\
\hline$C(26)-C(25)-C(24)$ & $108.5(3)$ \\
\hline $\mathrm{C}(31)-\mathrm{C}(25)-\mathrm{C}(24)$ & $130.4(3)$ \\
\hline$C(28)-C(26)-C(25)$ & $122.6(3)$ \\
\hline $\mathrm{C}(28)-\mathrm{C}(26)-\mathrm{C}(27)$ & $129.5(3)$ \\
\hline $\mathrm{C}(25)-\mathrm{C}(26)-\mathrm{C}(27)$ & $107.9(3)$ \\
\hline $\mathrm{O}(3)-\mathrm{C}(27)-\mathrm{N}(1)$ & $124.3(3)$ \\
\hline $\mathrm{O}(3)-\mathrm{C}(27)-\mathrm{C}(26)$ & $129.8(3)$ \\
\hline $\mathrm{N}(1)-\mathrm{C}(27)-\mathrm{C}(26)$ & $106.0(3)$ \\
\hline
\end{tabular}




$\begin{array}{ll}\mathrm{C}(26)-\mathrm{C}(28)-\mathrm{C}(29) & 116.5(3) \\ \mathrm{C}(26)-\mathrm{C}(28)-\mathrm{H}(28) & 121.7 \\ \mathrm{C}(29)-\mathrm{C}(28)-\mathrm{H}(28) & 121.7 \\ \mathrm{C}(30)-\mathrm{C}(29)-\mathrm{C}(28) & 121.4(3) \\ \mathrm{C}(30)-\mathrm{C}(29)-\mathrm{H}(29) & 119.3 \\ \mathrm{C}(28)-\mathrm{C}(29)-\mathrm{H}(29) & 119.3 \\ \mathrm{C}(31)-\mathrm{C}(30)-\mathrm{C}(29) & 121.7(3) \\ \mathrm{C}(31)-\mathrm{C}(30)-\mathrm{H}(30) & 119.1 \\ \mathrm{C}(29)-\mathrm{C}(30)-\mathrm{H}(30) & 119.1 \\ \mathrm{C}(30)-\mathrm{C}(31)-\mathrm{C}(25) & 116.7(3) \\ \mathrm{C}(30)-\mathrm{C}(31)-\mathrm{H}(31) & 121.7 \\ \mathrm{C}(25)-\mathrm{C}(31)-\mathrm{H}(31) & 121.7\end{array}$

Symmetry transformations used to generate equivalent atoms:

Table 4. Anisotropic displacement parameters $\left(\AA^{2} \times 10^{3}\right)$ for $\mathbf{3 o} .^{a}$

\begin{tabular}{|c|c|c|c|c|c|c|}
\hline & U11 & U22 & U33 & U23 & U13 & U12 \\
\hline $\mathrm{N}(1)$ & $36(1)$ & $44(2)$ & $45(1)$ & $-15(1)$ & $0(1)$ & $0(1)$ \\
\hline $\mathrm{O}(1)$ & $76(2)$ & $45(1)$ & $47(1)$ & $-23(1)$ & $-1(1)$ & $3(1)$ \\
\hline $\mathrm{O}(2)$ & $44(1)$ & $63(2)$ & $113(2)$ & $-19(2)$ & $-23(1)$ & $8(1)$ \\
\hline $\mathrm{O}(3)$ & $47(1)$ & $56(2)$ & $60(1)$ & $-11(1)$ & $-17(1)$ & 2(1) \\
\hline $\operatorname{Se}(1)$ & $57(1)$ & $51(1)$ & $57(1)$ & $-19(1)$ & $5(1)$ & $-11(1)$ \\
\hline$C(1)$ & $71(2)$ & $52(2)$ & $57(2)$ & $-18(2)$ & $8(2)$ & $3(2)$ \\
\hline $\mathrm{C}(2)$ & $115(4)$ & $56(2)$ & $59(3)$ & $-21(2)$ & $21(2)$ & $3(2)$ \\
\hline C(3) & $145(4)$ & $57(3)$ & $50(2)$ & $-24(2)$ & $-5(3)$ & $-2(3)$ \\
\hline $\mathrm{C}(4)$ & $96(3)$ & $75(3)$ & $61(2)$ & $-30(2)$ & $-17(2)$ & $-1(2)$ \\
\hline $\mathrm{C}(5)$ & 61(2) & $65(2)$ & $57(2)$ & $-28(2)$ & $-6(2)$ & $1(2)$ \\
\hline$C(6)$ & $56(2)$ & $40(2)$ & $42(2)$ & $-15(2)$ & $5(2)$ & $-5(2)$ \\
\hline$C(7)$ & $42(2)$ & $39(2)$ & $47(2)$ & $-13(2)$ & 2(1) & $3(1)$ \\
\hline$C(8)$ & $45(2)$ & $39(2)$ & $48(2)$ & $-17(2)$ & $-4(2)$ & $2(1)$ \\
\hline $\mathrm{C}(9)$ & $59(2)$ & $45(2)$ & $48(2)$ & $-16(2)$ & $1(2)$ & $-4(2)$ \\
\hline $\mathrm{C}(10)$ & 78(2) & $65(2)$ & $59(2)$ & $-30(2)$ & $-18(2)$ & $11(2)$ \\
\hline$C(11)$ & $70(2)$ & $52(2)$ & $48(2)$ & $-29(2)$ & $-18(2)$ & $7(2)$ \\
\hline $\mathrm{C}(12)$ & 76(3) & $64(2)$ & $44(2)$ & $-25(2)$ & $-11(2)$ & $10(2)$ \\
\hline $\mathrm{C}(13)$ & $88(3)$ & $76(3)$ & $69(3)$ & $-36(2)$ & $-6(2)$ & $22(3)$ \\
\hline$C(14)$ & $130(4)$ & $59(3)$ & $75(3)$ & $-30(2)$ & $-17(3)$ & $26(3)$ \\
\hline$C(15)$ & $122(4)$ & $57(3)$ & $82(3)$ & $-23(2)$ & $-3(3)$ & $-5(3)$ \\
\hline$C(16)$ & $73(3)$ & $78(3)$ & $69(2)$ & $-39(2)$ & $-1(2)$ & $-2(2)$ \\
\hline $\mathrm{C}(17)$ & $84(3)$ & 111(4) & 77(3) & $-27(3)$ & $3(2)$ & $-6(3)$ \\
\hline$C(18)$ & $63(2)$ & $35(2)$ & $48(2)$ & $-7(2)$ & $9(2)$ & $-7(2)$ \\
\hline$C(19)$ & $83(3)$ & $56(2)$ & $50(2)$ & $-12(2)$ & $-1(2)$ & $1(2)$ \\
\hline $\mathrm{C}(20)$ & 104(3) & 70(3) & 66(3) & $-11(2)$ & $-21(3)$ & $-1(3)$ \\
\hline $\mathrm{C}(21)$ & $78(3)$ & $61(3)$ & $92(3)$ & $-10(2)$ & $-4(3)$ & $8(2)$ \\
\hline$C(22)$ & $85(3)$ & $60(3)$ & 79(3) & $-18(2)$ & $11(2)$ & $11(2)$ \\
\hline
\end{tabular}




$\begin{array}{llllrrr}\mathrm{C}(23) & 82(3) & 52(2) & 56(2) & -18(2) & 4(2) & 3(2) \\ \mathrm{C}(24) & 36(2) & 57(2) & 50(2) & -17(2) & -3(2) & 4(2) \\ \mathrm{C}(25) & 41(2) & 54(2) & 41(2) & -19(2) & 3(1) & -2(2) \\ \mathrm{C}(26) & 46(2) & 45(2) & 36(2) & -12(2) & 2(1) & -3(2) \\ \mathrm{C}(27) & 44(2) & 50(2) & 36(2) & -14(2) & -1(2) & 0(2) \\ \mathrm{C}(28) & 65(2) & 49(2) & 50(2) & -12(2) & -4(2) & -7(2) \\ \mathrm{C}(29) & 92(3) & 49(2) & 55(2) & -12(2) & 8(2) & -12(2) \\ \mathrm{C}(30) & 74(3) & 75(3) & 63(2) & -34(2) & 16(2) & -36(2) \\ \mathrm{C}(31) & 48(2) & 76(3) & 55(2) & -30(2) & 1(2) & -12(2)\end{array}$

${ }^{a}$ The anisotropic displacement factor exponent takes the form: $-2 \pi^{2}\left[\mathrm{~h}^{2} \mathrm{a}^{*^{2}} \mathrm{U}^{11}+\ldots\right.$ $+2 \mathrm{~h} \mathrm{k} \mathrm{a}^{*} \mathrm{~b}^{*} \mathrm{U}^{12}$ ]

Table 5. Hydrogen coordinates ( $\times 10^{4}$ ) and isotropic displacement

\begin{tabular}{|c|c|c|c|c|}
\hline & $\mathrm{x}$ & $\mathrm{y}$ & z & $\mathrm{U}(\mathrm{eq})$ \\
\hline $\mathrm{H}(1)$ & 4511 & 1090 & 715 & 72 \\
\hline $\mathrm{H}(2)$ & 4083 & 748 & -736 & 92 \\
\hline $\mathrm{H}(3)$ & 1061 & 1011 & -1418 & 99 \\
\hline $\mathrm{H}(4)$ & -1512 & 1629 & -677 & 90 \\
\hline $\mathrm{H}(5)$ & -1099 & 1984 & 766 & 71 \\
\hline $\mathrm{H}(7)$ & 3201 & 1342 & 2138 & 51 \\
\hline $\mathrm{H}(8)$ & -615 & 2119 & 2253 & 52 \\
\hline $\mathrm{H}(9 \mathrm{~A})$ & 44 & 1726 & 3989 & 60 \\
\hline $\mathrm{H}(9 \mathrm{~B})$ & 2260 & 1859 & 3631 & 60 \\
\hline $\mathrm{H}(10 \mathrm{~A})$ & 2647 & 3491 & 3997 & 77 \\
\hline $\mathrm{H}(10 \mathrm{~B})$ & 635 & 3130 & 4557 & 77 \\
\hline $\mathrm{H}(13)$ & -3160 & 6075 & 3844 & 90 \\
\hline $\mathrm{H}(14)$ & -955 & 7328 & 3110 & 103 \\
\hline $\mathrm{H}(15)$ & 2172 & 6907 & 2723 & 103 \\
\hline $\mathrm{H}(16)$ & 3122 & 5225 & 3111 & 84 \\
\hline $\mathrm{H}(17 \mathrm{~A})$ & -4097 & 4381 & 4496 & 137 \\
\hline $\mathrm{H}(17 \mathrm{~B})$ & -2413 & 3640 & 4991 & 137 \\
\hline $\mathrm{H}(17 \mathrm{C})$ & -3070 & 3652 & 3962 & 137 \\
\hline $\mathrm{H}(19)$ & 1820 & -293 & 4775 & 77 \\
\hline $\mathrm{H}(20)$ & 4608 & -1231 & 5300 & 98 \\
\hline $\mathrm{H}(21)$ & 6443 & -1940 & 4278 & 96 \\
\hline $\mathrm{H}(22)$ & 5450 & -1717 & 2733 & 91 \\
\hline $\mathrm{H}(23)$ & 2645 & -803 & 2202 & 76 \\
\hline $\mathrm{H}(28)$ & 2021 & 5922 & 465 & 66 \\
\hline $\mathrm{H}(29)$ & 4612 & 6941 & 623 & 80 \\
\hline $\mathrm{H}(30)$ & 7414 & 6222 & 1371 & 81 \\
\hline $\mathrm{H}(31)$ & 7752 & 4464 & 2007 & 68 \\
\hline
\end{tabular}


Table 6. Torsion angles $\left[^{\circ}\right]$ for 30 .

\begin{tabular}{|c|c|}
\hline $\mathrm{C}(6)-\mathrm{C}(1)-\mathrm{C}(2)-\mathrm{C}(3)$ & $-0.5(5)$ \\
\hline $\mathrm{C}(1)-\mathrm{C}(2)-\mathrm{C}(3)-\mathrm{C}(4)$ & $0.4(6)$ \\
\hline $\mathrm{C}(2)-\mathrm{C}(3)-\mathrm{C}(4)-\mathrm{C}(5)$ & $-0.1(6)$ \\
\hline $\mathrm{C}(3)-\mathrm{C}(4)-\mathrm{C}(5)-\mathrm{C}(6)$ & $0.0(6)$ \\
\hline $\mathrm{C}(4)-\mathrm{C}(5)-\mathrm{C}(6)-\mathrm{C}(1)$ & $-0.1(5)$ \\
\hline$C(4)-C(5)-C(6)-C(7)$ & $178.1(3)$ \\
\hline$C(2)-C(1)-C(6)-C(5)$ & $0.3(5)$ \\
\hline $\mathrm{C}(2)-\mathrm{C}(1)-\mathrm{C}(6)-\mathrm{C}(7)$ & $-178.0(3)$ \\
\hline $\mathrm{C}(24)-\mathrm{N}(1)-\mathrm{C}(7)-\mathrm{C}(6)$ & $-123.1(3)$ \\
\hline $\mathrm{C}(27)-\mathrm{N}(1)-\mathrm{C}(7)-\mathrm{C}(6)$ & $65.8(3)$ \\
\hline $\mathrm{C}(24)-\mathrm{N}(1)-\mathrm{C}(7)-\mathrm{C}(8)$ & $107.0(3)$ \\
\hline $\mathrm{C}(27)-\mathrm{N}(1)-\mathrm{C}(7)-\mathrm{C}(8)$ & $-64.1(3)$ \\
\hline $\mathrm{C}(5)-\mathrm{C}(6)-\mathrm{C}(7)-\mathrm{N}(1)$ & $-94.2(3)$ \\
\hline $\mathrm{C}(1)-\mathrm{C}(6)-\mathrm{C}(7)-\mathrm{N}(1)$ & $84.0(3)$ \\
\hline $\mathrm{C}(5)-\mathrm{C}(6)-\mathrm{C}(7)-\mathrm{C}(8)$ & $32.5(4)$ \\
\hline $\mathrm{C}(1)-\mathrm{C}(6)-\mathrm{C}(7)-\mathrm{C}(8)$ & $-149.3(3)$ \\
\hline $\mathrm{N}(1)-\mathrm{C}(7)-\mathrm{C}(8)-\mathrm{C}(9)$ & $-47.8(3)$ \\
\hline $\mathrm{C}(6)-\mathrm{C}(7)-\mathrm{C}(8)-\mathrm{C}(9)$ & $-173.9(3)$ \\
\hline $\mathrm{N}(1)-\mathrm{C}(7)-\mathrm{C}(8)-\mathrm{Se}(1)$ & $-173.57(19)$ \\
\hline $\mathrm{C}(6)-\mathrm{C}(7)-\mathrm{C}(8)-\mathrm{Se}(1)$ & $60.2(3)$ \\
\hline $\mathrm{C}(18)-\mathrm{Se}(1)-\mathrm{C}(8)-\mathrm{C}(9)$ & $-72.0(2)$ \\
\hline $\mathrm{C}(18)-\mathrm{Se}(1)-\mathrm{C}(8)-\mathrm{C}(7)$ & $55.5(2)$ \\
\hline $\mathrm{C}(10)-\mathrm{O}(1)-\mathrm{C}(9)-\mathrm{C}(8)$ & $-175.7(3)$ \\
\hline $\mathrm{C}(7)-\mathrm{C}(8)-\mathrm{C}(9)-\mathrm{O}(1)$ & $81.0(3)$ \\
\hline $\mathrm{Se}(1)-\mathrm{C}(8)-\mathrm{C}(9)-\mathrm{O}(1)$ & $-153.0(2)$ \\
\hline $\mathrm{C}(9)-\mathrm{O}(1)-\mathrm{C}(10)-\mathrm{C}(11)$ & $-168.1(3)$ \\
\hline $\mathrm{O}(1)-\mathrm{C}(10)-\mathrm{C}(11)-\mathrm{C}(16)$ & $-96.8(4)$ \\
\hline $\mathrm{O}(1)-\mathrm{C}(10)-\mathrm{C}(11)-\mathrm{C}(12)$ & $83.2(4)$ \\
\hline $\mathrm{C}(16)-\mathrm{C}(11)-\mathrm{C}(12)-\mathrm{C}(13)$ & $-1.1(5)$ \\
\hline $\mathrm{C}(10)-\mathrm{C}(11)-\mathrm{C}(12)-\mathrm{C}(13)$ & $178.9(3)$ \\
\hline $\mathrm{C}(16)-\mathrm{C}(11)-\mathrm{C}(12)-\mathrm{C}(17)$ & $178.4(3)$ \\
\hline $\mathrm{C}(10)-\mathrm{C}(11)-\mathrm{C}(12)-\mathrm{C}(17)$ & $-1.5(5)$ \\
\hline $\mathrm{C}(11)-\mathrm{C}(12)-\mathrm{C}(13)-\mathrm{C}(14)$ & $1.0(5)$ \\
\hline $\mathrm{C}(17)-\mathrm{C}(12)-\mathrm{C}(13)-\mathrm{C}(14)$ & $-178.6(4)$ \\
\hline$C(12)-C(13)-C(14)-C(15)$ & $0.1(6)$ \\
\hline$C(13)-C(14)-C(15)-C(16)$ & $-1.0(6)$ \\
\hline$C(14)-C(15)-C(16)-C(11)$ & $0.9(6)$ \\
\hline $\mathrm{C}(12)-\mathrm{C}(11)-\mathrm{C}(16)-\mathrm{C}(15)$ & $0.2(5)$ \\
\hline$C(10)-C(11)-C(16)-C(15)$ & $-179.8(3)$ \\
\hline $\mathrm{C}(8)-\mathrm{Se}(1)-\mathrm{C}(18)-\mathrm{C}(19)$ & $85.8(3)$ \\
\hline
\end{tabular}




$\begin{array}{lr}\mathrm{C}(8)-\mathrm{Se}(1)-\mathrm{C}(18)-\mathrm{C}(23) & -98.0(3) \\ \mathrm{C}(23)-\mathrm{C}(18)-\mathrm{C}(19)-\mathrm{C}(20) & 0.3(5) \\ \mathrm{Se}(1)-\mathrm{C}(18)-\mathrm{C}(19)-\mathrm{C}(20) & 176.6(3) \\ \mathrm{C}(18)-\mathrm{C}(19)-\mathrm{C}(20)-\mathrm{C}(21) & 0.3(6) \\ \mathrm{C}(19)-\mathrm{C}(20)-\mathrm{C}(21)-\mathrm{C}(22) & -0.4(6) \\ \mathrm{C}(20)-\mathrm{C}(21)-\mathrm{C}(22)-\mathrm{C}(23) & -0.1(6) \\ \mathrm{C}(21)-\mathrm{C}(22)-\mathrm{C}(23)-\mathrm{C}(18) & 0.7(6) \\ \mathrm{C}(19)-\mathrm{C}(18)-\mathrm{C}(23)-\mathrm{C}(22) & -0.8(5) \\ \mathrm{Se}(1)-\mathrm{C}(18)-\mathrm{C}(23)-\mathrm{C}(22) & -177.1(3) \\ \mathrm{C}(27)-\mathrm{N}(1)-\mathrm{C}(24)-\mathrm{O}(2) & 176.0(3) \\ \mathrm{C}(7)-\mathrm{N}(1)-\mathrm{C}(24)-\mathrm{O}(2) & 3.7(4) \\ \mathrm{C}(27)-\mathrm{N}(1)-\mathrm{C}(24)-\mathrm{C}(25) & -3.2(3) \\ \mathrm{C}(7)-\mathrm{N}(1)-\mathrm{C}(24)-\mathrm{C}(25) & -175.5(2) \\ \mathrm{O}(2)-\mathrm{C}(24)-\mathrm{C}(25)-\mathrm{C}(26) & -177.1(3) \\ \mathrm{N}(1)-\mathrm{C}(24)-\mathrm{C}(25)-\mathrm{C}(26) & 2.0(3) \\ \mathrm{O}(2)-\mathrm{C}(24)-\mathrm{C}(25)-\mathrm{C}(31) & 3.3(5) \\ \mathrm{N}(1)-\mathrm{C}(24)-\mathrm{C}(25)-\mathrm{C}(31) & -177.6(3) \\ \mathrm{C}(31)-\mathrm{C}(25)-\mathrm{C}(26)-\mathrm{C}(28) & -0.1(4) \\ \mathrm{C}(24)-\mathrm{C}(25)-\mathrm{C}(26)-\mathrm{C}(28) & -179.7(3) \\ \mathrm{C}(31)-\mathrm{C}(25)-\mathrm{C}(26)-\mathrm{C}(27) & 179.5(3) \\ \mathrm{C}(24)-\mathrm{C}(25)-\mathrm{C}(26)-\mathrm{C}(27) & -0.1(3) \\ \mathrm{C}(24)-\mathrm{N}(1)-\mathrm{C}(27)-\mathrm{O}(3) & -177.1(3) \\ \mathrm{C}(7)-\mathrm{N}(1)-\mathrm{C}(27)-\mathrm{O}(3) & -5.1(4) \\ \mathrm{C}(24)-\mathrm{N}(1)-\mathrm{C}(27)-\mathrm{C}(26) & 3.1(3) \\ \mathrm{C}(7)-\mathrm{N}(1)-\mathrm{C}(27)-\mathrm{C}(26) & 175.1(2) \\ \mathrm{C}(28)-\mathrm{C}(26)-\mathrm{C}(27)-\mathrm{O}(3) & -2.0(5) \\ \mathrm{C}(25)-\mathrm{C}(26)-\mathrm{C}(27)-\mathrm{O}(3) & 178.4(3) \\ \mathrm{C}(28)-\mathrm{C}(26)-\mathrm{C}(27)-\mathrm{N}(1) & 177.7(3) \\ \mathrm{C}(25)-\mathrm{C}(26)-\mathrm{C}(27)-\mathrm{N}(1) & -1.8(3) \\ \mathrm{C}(25)-\mathrm{C}(26)-\mathrm{C}(28)-\mathrm{C}(29) & 0.0(4) \\ \mathrm{C}(27)-\mathrm{C}(26)-\mathrm{C}(28)-\mathrm{C}(29) & -0.3(3) \\ \mathrm{C}(26)-\mathrm{C}(28)-\mathrm{C}(29)-\mathrm{C}(30) & \\ \mathrm{C}(28)-\mathrm{C}(29)-\mathrm{C}(30)-\mathrm{C}(31) & -179.4(3) \\ \mathrm{C}(29)-\mathrm{C}(30)-\mathrm{C}(31)-\mathrm{C}(25) & \\ \mathrm{C}(26)-\mathrm{C}(25)-\mathrm{C}(31)-\mathrm{C}(30) & \\ \mathrm{C}(24)-\mathrm{C}(25)-\mathrm{C}(31)-\mathrm{C}(30) & \\ & \\ & \end{array}$

Symmetry transformations used to generate equivalent atoms:

Table 7. Hydrogen bonds for 30 [^ and $\left.{ }^{\circ}\right]$.

\begin{tabular}{lllll}
\hline D-H...A & d(D-H) & d(H...A $)$ & d(D...A $)$ & $<($ DHA $)$
\end{tabular}

\section{References}


(1) Bugarin, A.; Connell, B. T. Chem. Commun. 2011, 47, 7218.

(2) (a) Charette, A. B.; Molinaro, C.; Brochu, C. J. Am. Chem. Soc. 2001, 123, 12160. (b) Nicolaou, K. C.; Claremon, D. A.; Barnette, W. E.; Seitz, S. P. J. Am. Chem. Soc.1979, 101, 3704.

(3) Reich, H. J.; Renga, J. N.; Reich, I. L. J. Am. Chem. Soc. 1975, 97, 5434.

(4) Hori, T.; Sharpless, K. B. J. Org. Chem. 1979, 44, 4210.

(5) (a) Yang, M. H.; Yuan, C. Y.; Pan, Y.; Zhu, C. J. Chin. J. Chem. 2006, 24, 669. (b) Michelangelo, G.; Paolo, L. M.; Serena, R.; Francesca D. A.; Renato, N. Tetrahedron Asymmetry 2006, 17, 2713.

(6) Thvedt, T. H. K.; Fuglseth, E.; Sundby, E.; Hoff, B. H. Tetrahedron 2010, 66, 6733.

(7) Wirth, T. Organoselenium Chemistry: Synthesis and Reactions; Wiley-VCH: Weinheim, 2012.

\section{Copies of NMR Spectra for compounds}


${ }^{1} \mathrm{H}$ NMR $\left(300 \mathrm{MHz}, \mathrm{CDCl}_{3}\right)$<smiles>COc1ccc([C@H]([C@H](C)[CH+]c2ccccc2)N2C(=O)c3ccccc3C2=O)cc1OC</smiles>

3a

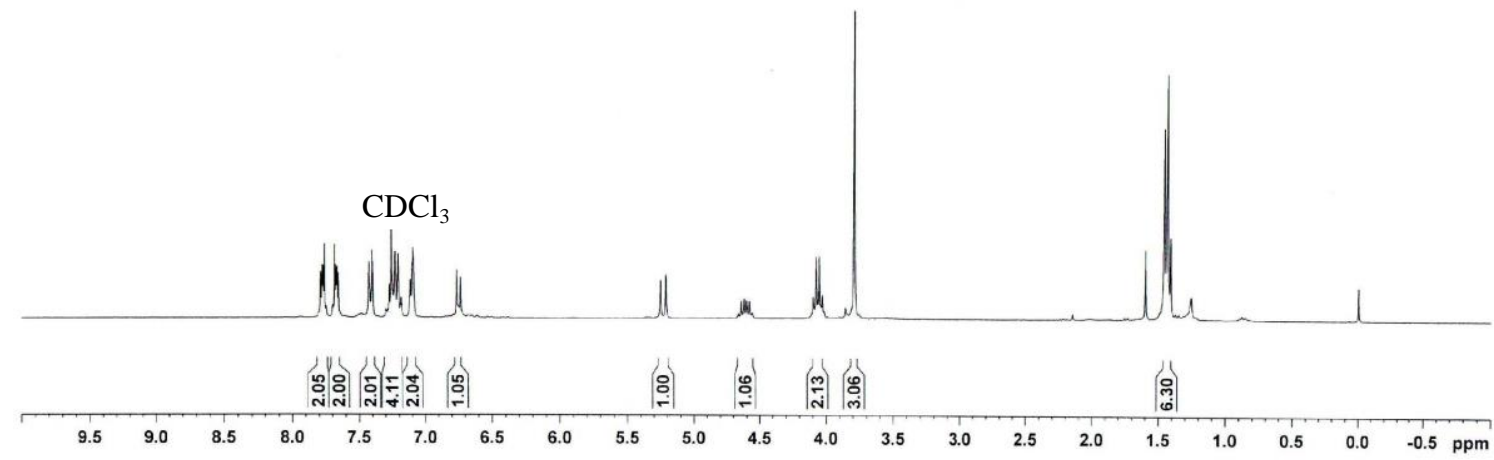

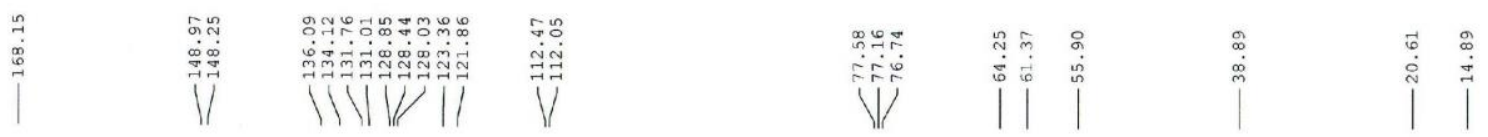

${ }^{13} \mathrm{C} \mathrm{NMR}\left(75 \mathrm{MHz}, \mathrm{CDCl}_{3}\right)$<smiles>COc1ccc([C@H](C(C)C(c2ccccc2)c2ccccc2)N2C(=O)c3ccccc3C2=O)c(OC)c1</smiles>

3a

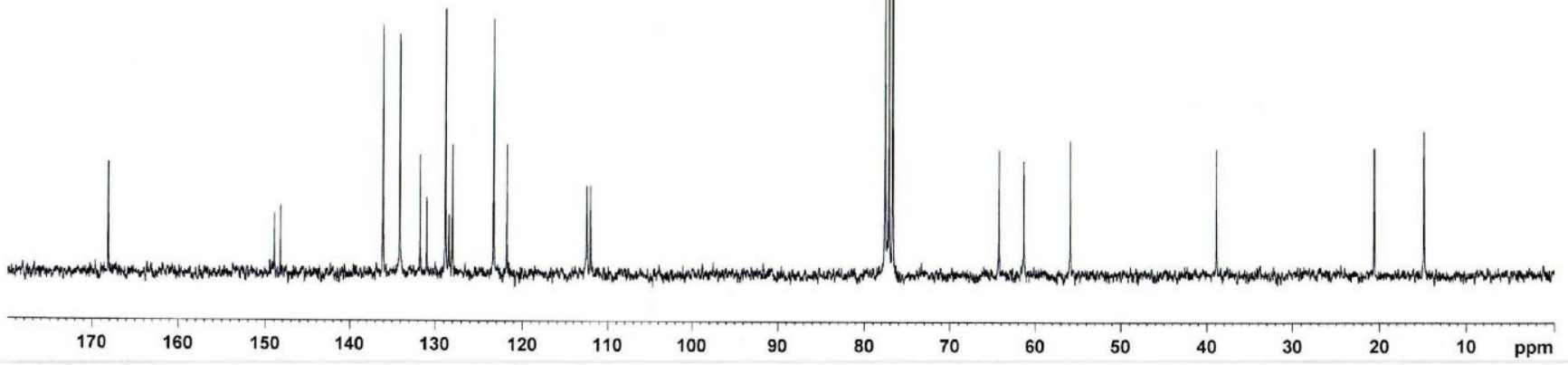


${ }^{1} \mathrm{H}$ NMR (300 MHz, $\mathrm{CDCl}_{3}$ )<smiles>O=C1c2ccccc2C(=O)N1C(C[Se]c1ccccc1)c1ccccc1</smiles>

3b
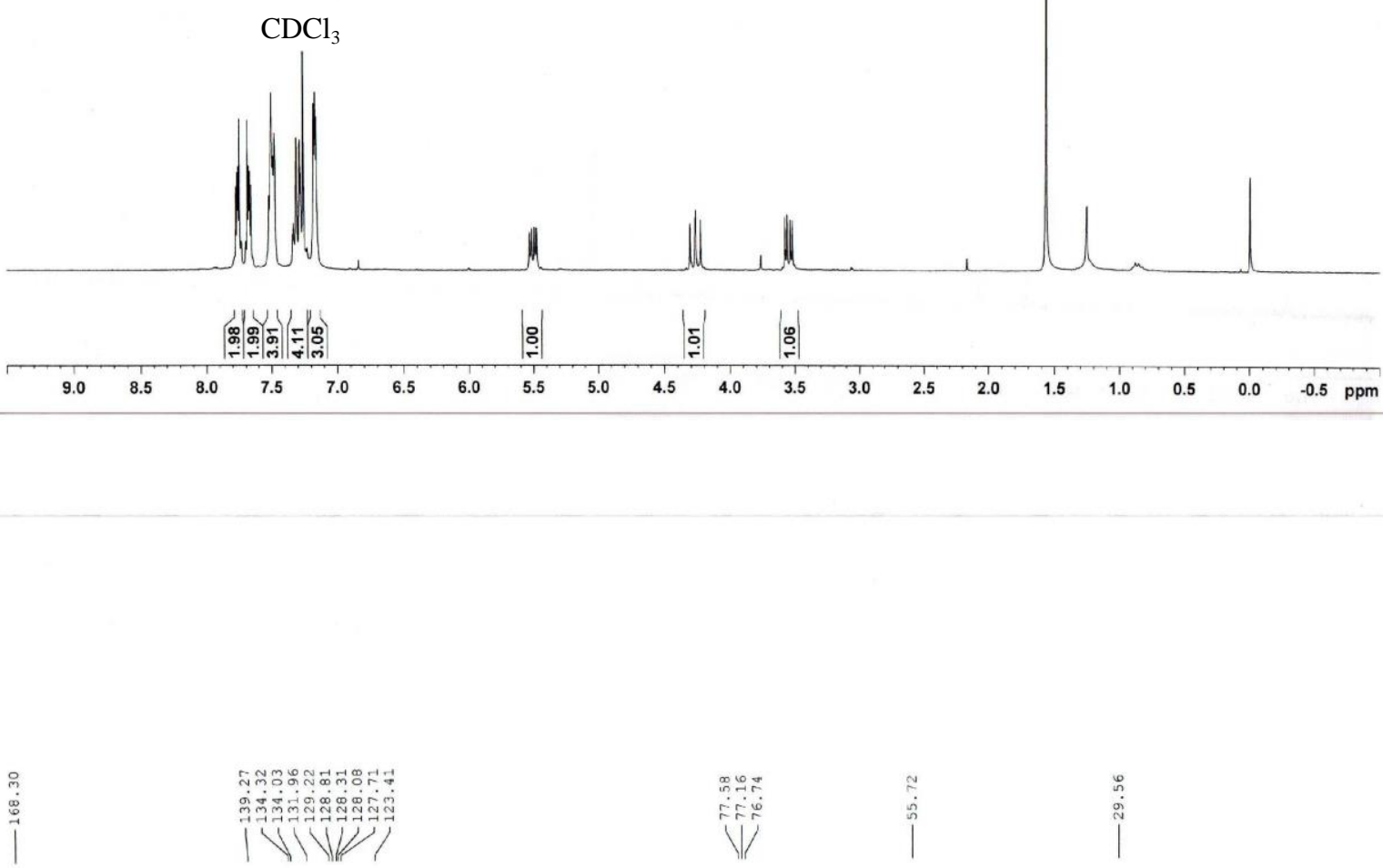

${ }^{13} \mathrm{C}$ NMR $\left(75 \mathrm{MHz}, \mathrm{CDCl}_{3}\right)$

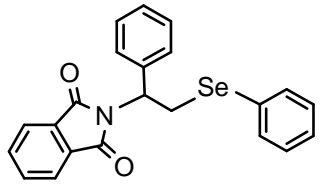

$3 b$

$\begin{array}{llll}170 & 160 & 150 & 140\end{array}$ 
${ }^{1} \mathrm{H}$ NMR $\left(300 \mathrm{MHz}, \mathrm{CDCl}_{3}\right)$
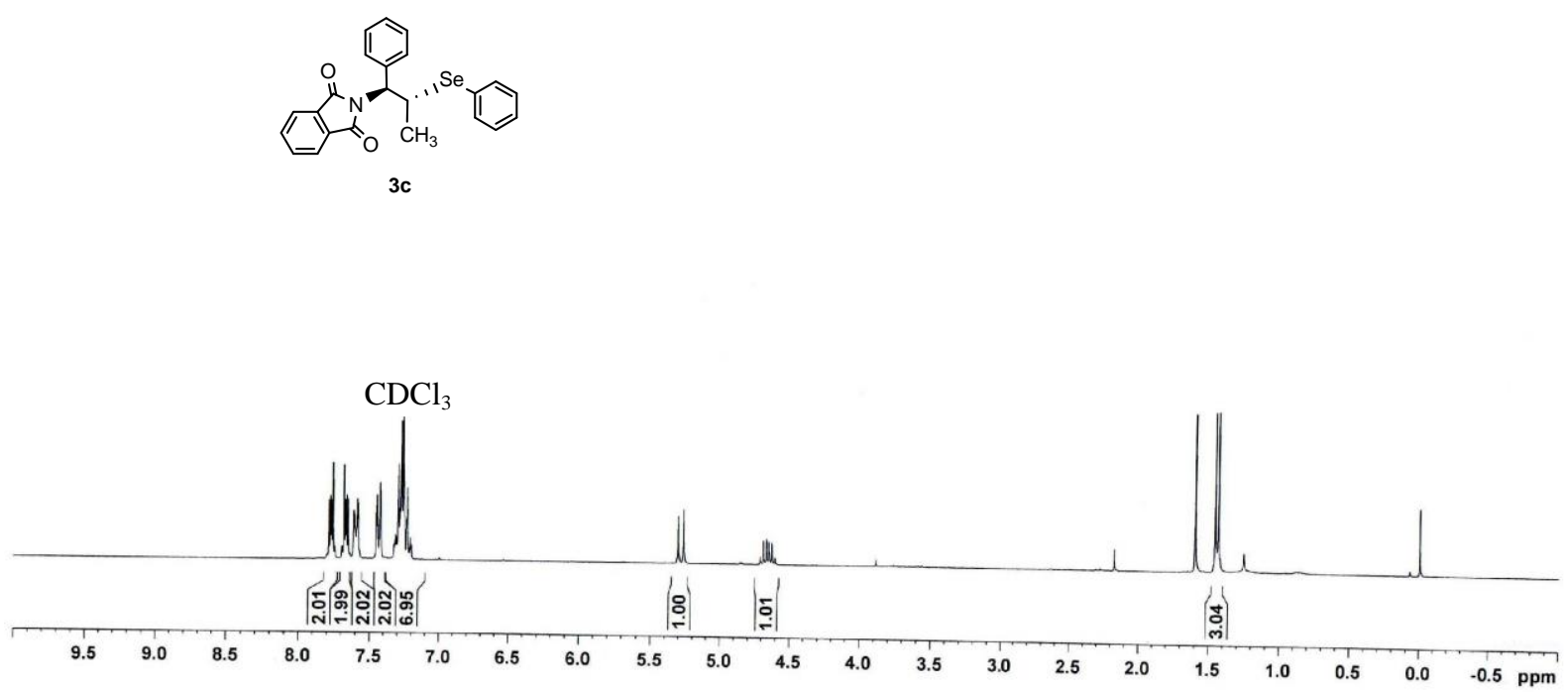

${ }^{13} \mathrm{C}$ NMR (75 MHz, $\mathrm{CDCl}_{3}$ )

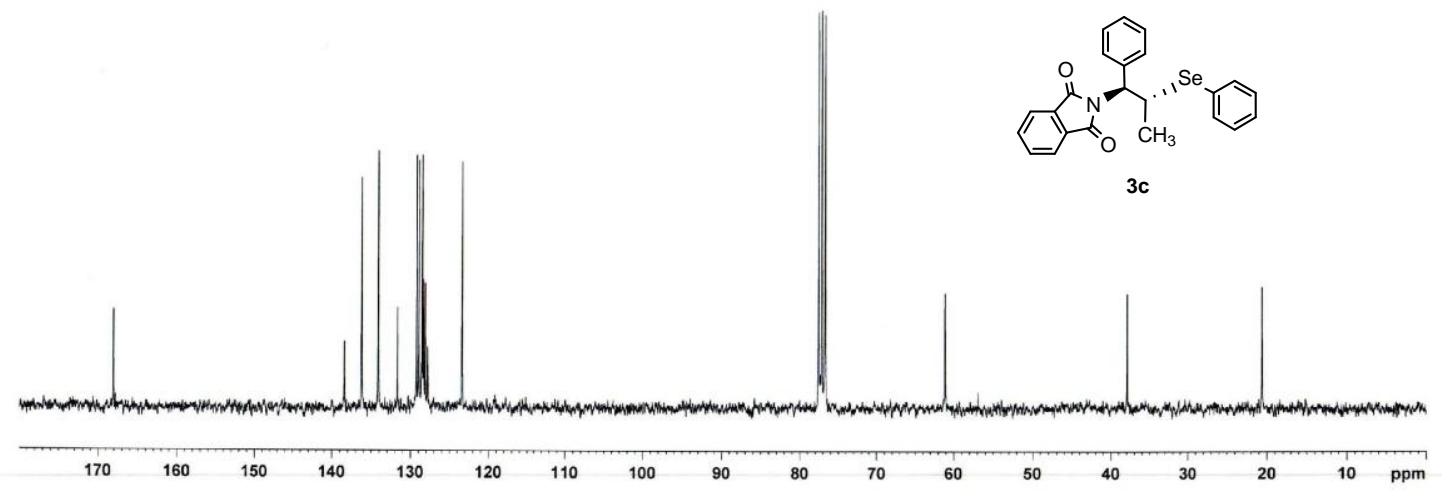


${ }^{1} \mathrm{H}$ NMR $\left(300 \mathrm{MHz}, \mathrm{CDCl}_{3}\right)$<smiles>Cc1ccc(C(Cc2ccccc2)N2C(=O)c3ccccc3C2=O)cc1</smiles>

3d
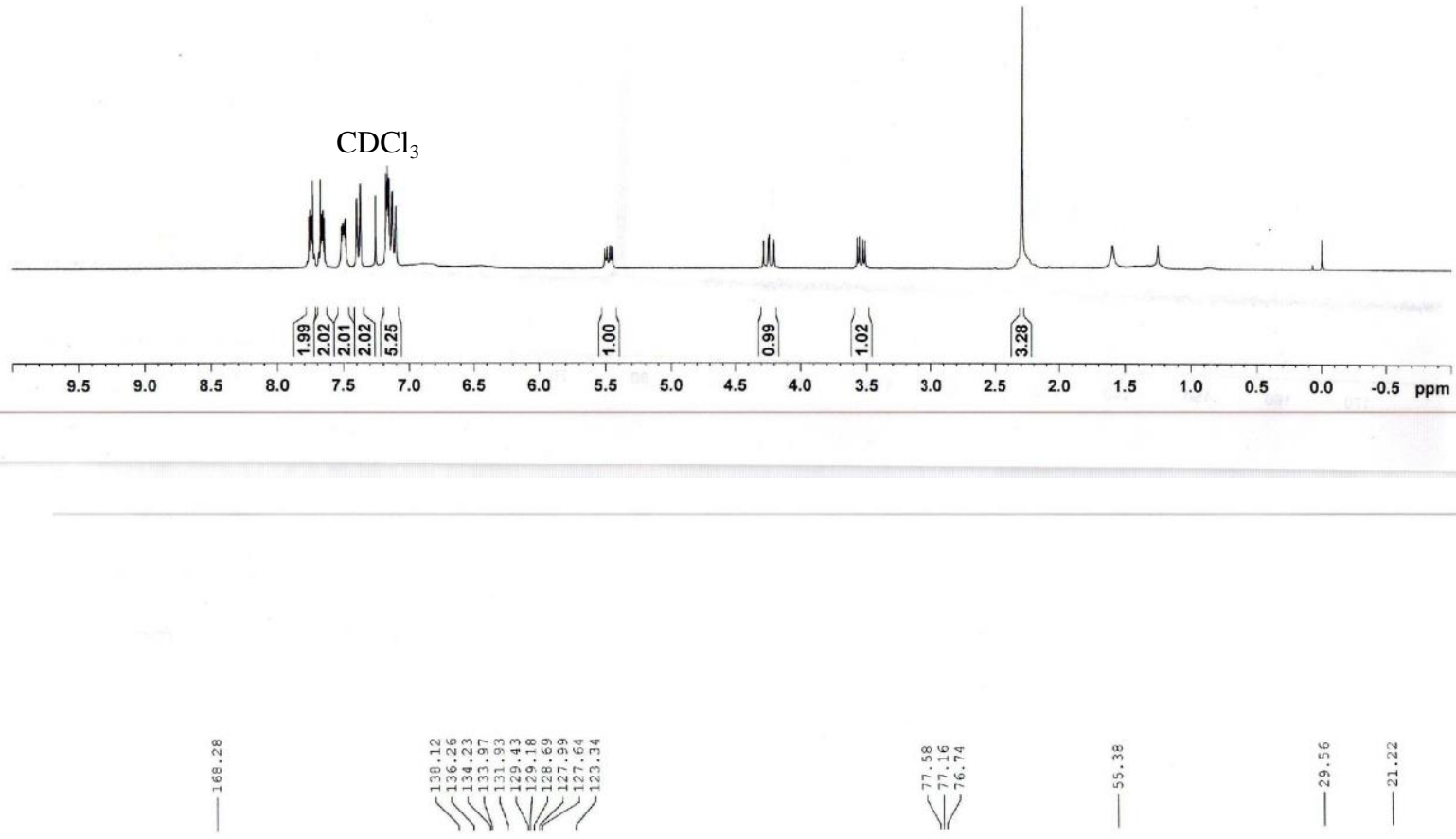

${ }^{13} \mathrm{C} \mathrm{NMR}\left(75 \mathrm{MHz}, \mathrm{CDCl}_{3}\right)$<smiles>Cc1ccc(C(C[Se]c2ccccc2)N2C(=O)c3ccccc3C2=O)cc1</smiles>

3d

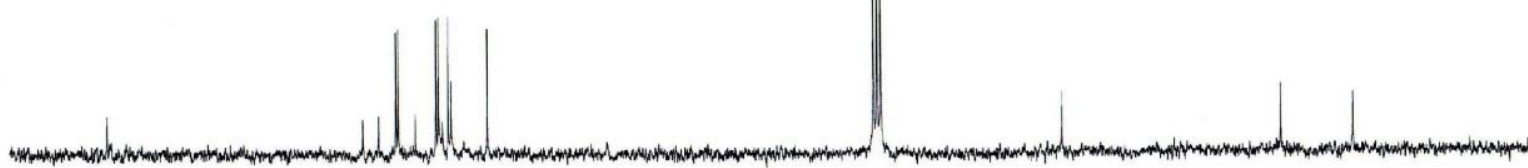

$\begin{array}{llllllllllllllllllll}170 & 160 & 150 & 140 & 130 & 120 & 110 & 100 & 90 & 80 & 70 & 60 & 50 & 40 & 30 & 20 & 10 & \mathrm{ppm}\end{array}$ 


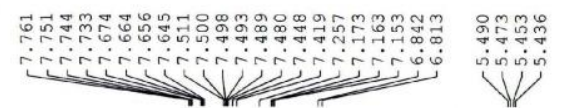

${ }^{1} \mathrm{H}$ NMR $\left(300 \mathrm{MHz}, \mathrm{CDCl}_{3}\right)$

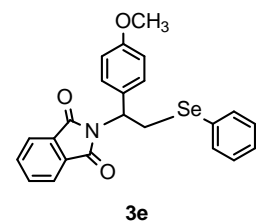

$3 e$

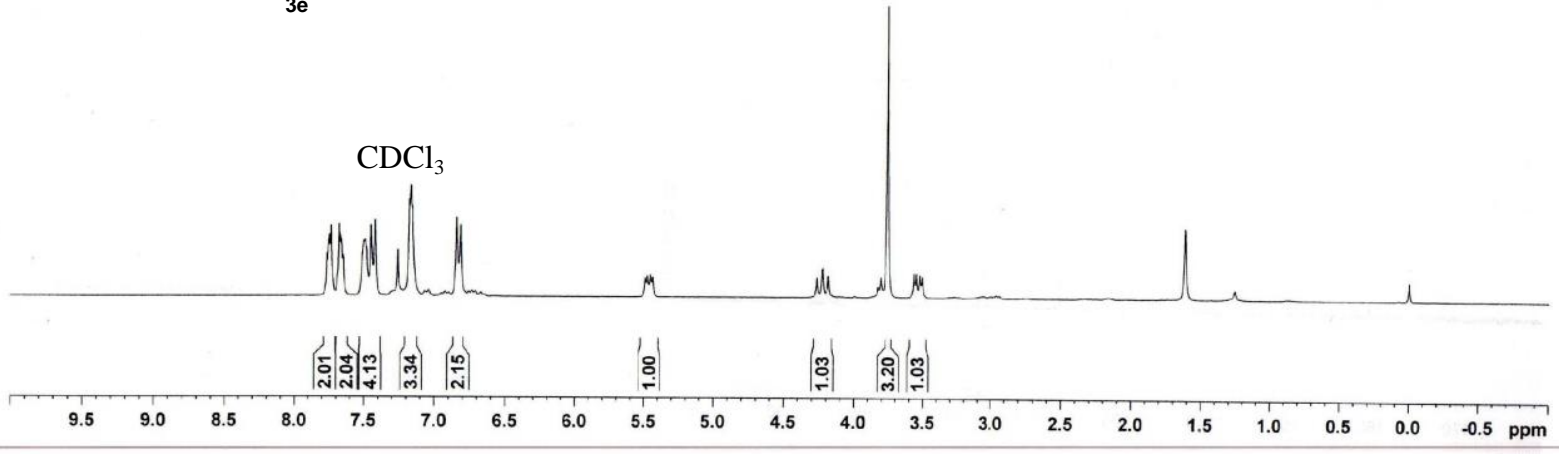

|

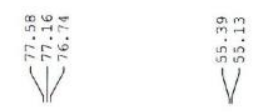

${ }^{13} \mathrm{C}$ NMR $\left(75 \mathrm{MHz}, \mathrm{CDCl}_{3}\right)$<smiles>COc1ccc(C(C[Se]c2ccccc2)n2c(=O)c3ccccc3c(=O)n2-c2ccccc2)cc1</smiles>

3e

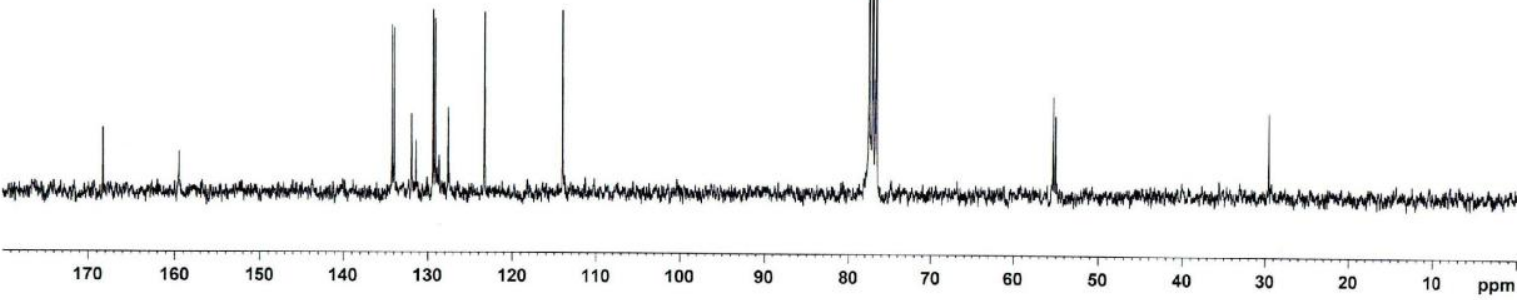



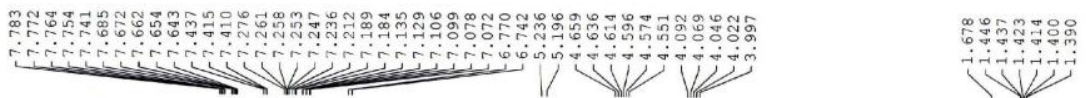

:

${ }^{1} \mathrm{H}$ NMR $\left(300 \mathrm{MHz}, \mathrm{CDCl}_{3}\right)$
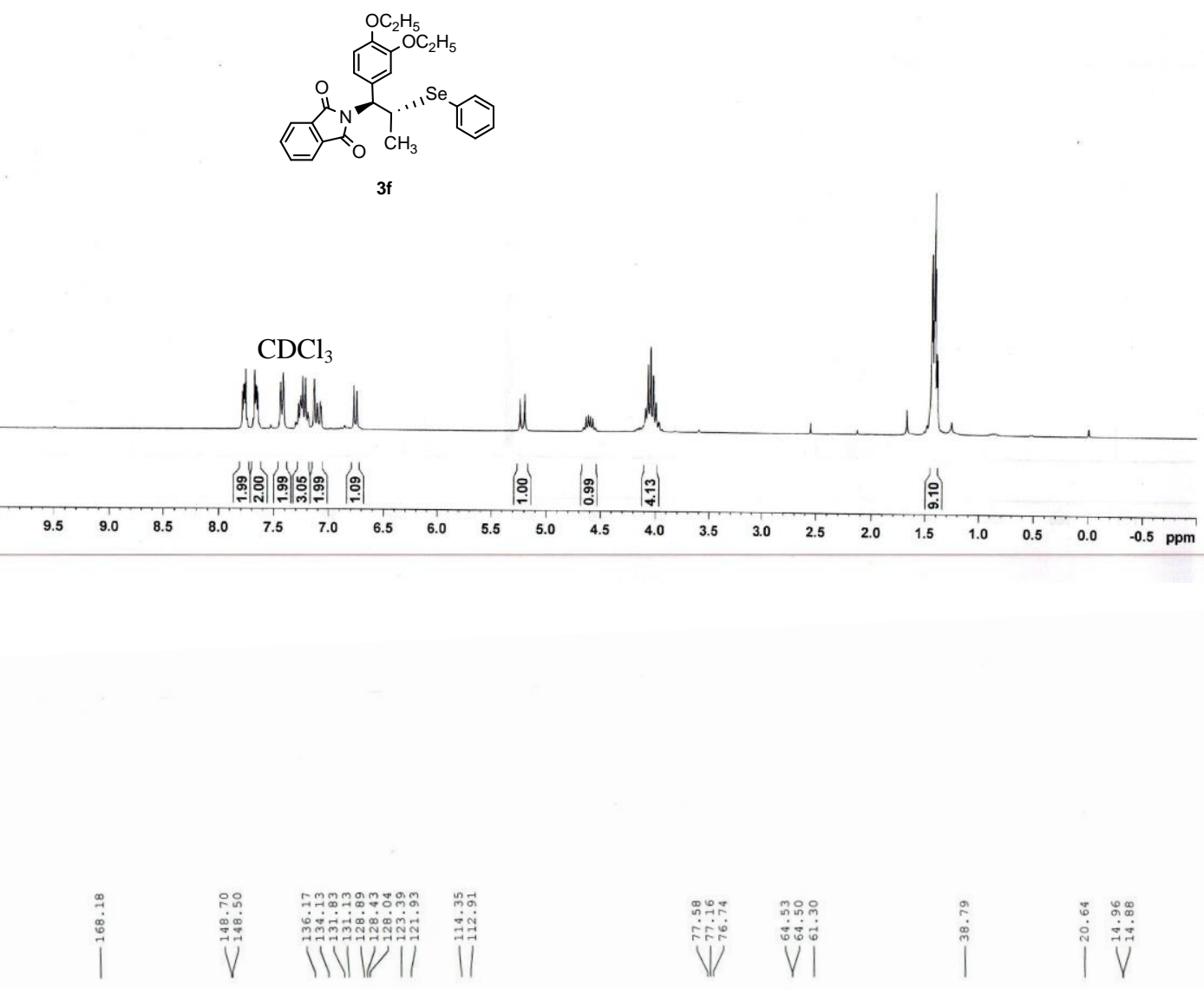

${ }^{13} \mathrm{C}$ NMR $\left(75 \mathrm{MHz}, \mathrm{CDCl}_{3}\right)$

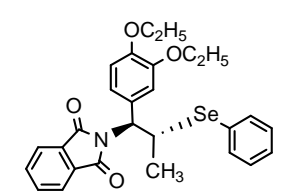

$3 f$

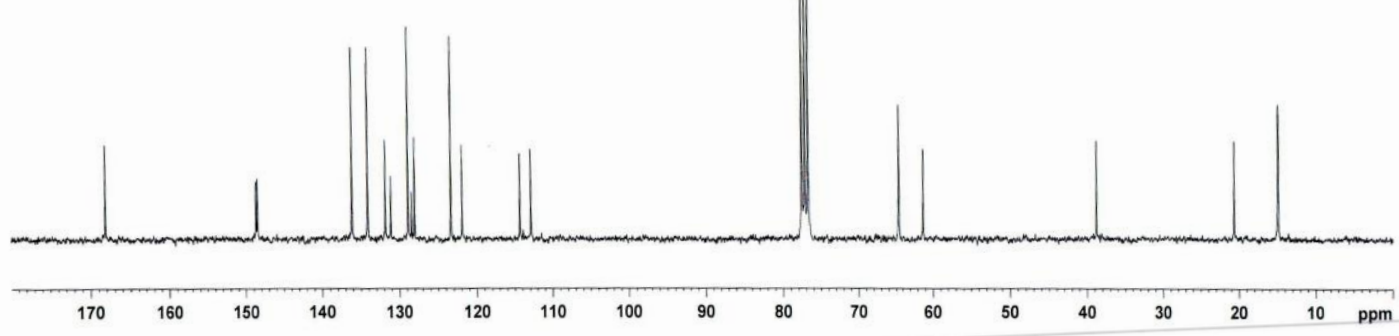

S27 
${ }^{1} \mathrm{H}$ NMR $\left(300 \mathrm{MHz}, \mathrm{CDCl}_{3}\right)$

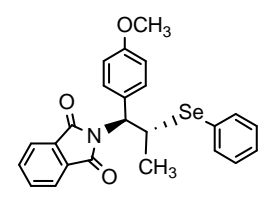

$3 g$

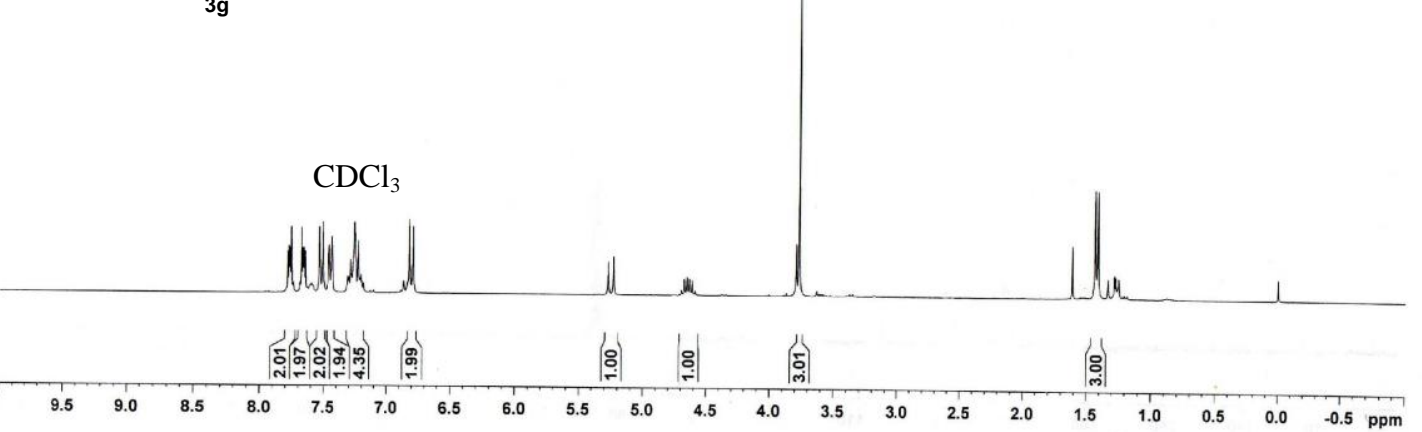

$\mid$

${ }^{13} \mathrm{C}$ NMR $\left(75 \mathrm{MHz}, \mathrm{CDCl}_{3}\right)$

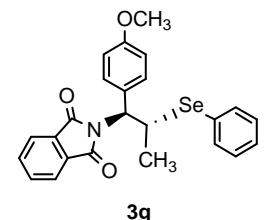

$3 g$

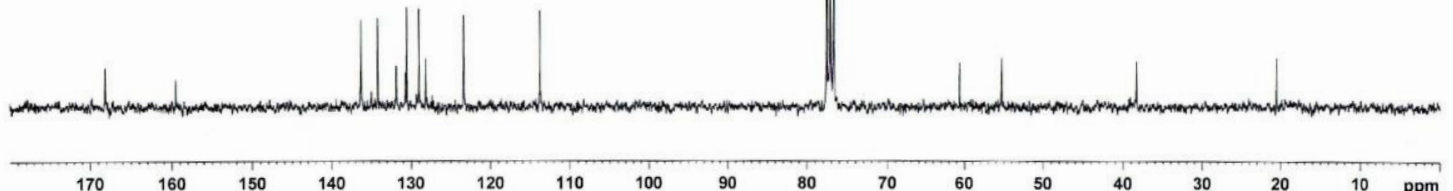



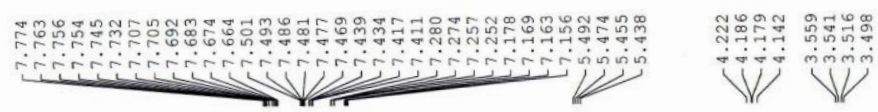

$\stackrel{\circ}{\stackrel{2}{i}}$

$\stackrel{\circ}{\dot{1}}$

${ }^{1} \mathrm{H}$ NMR (300 MHz, $\mathrm{CDCl}_{3}$ )
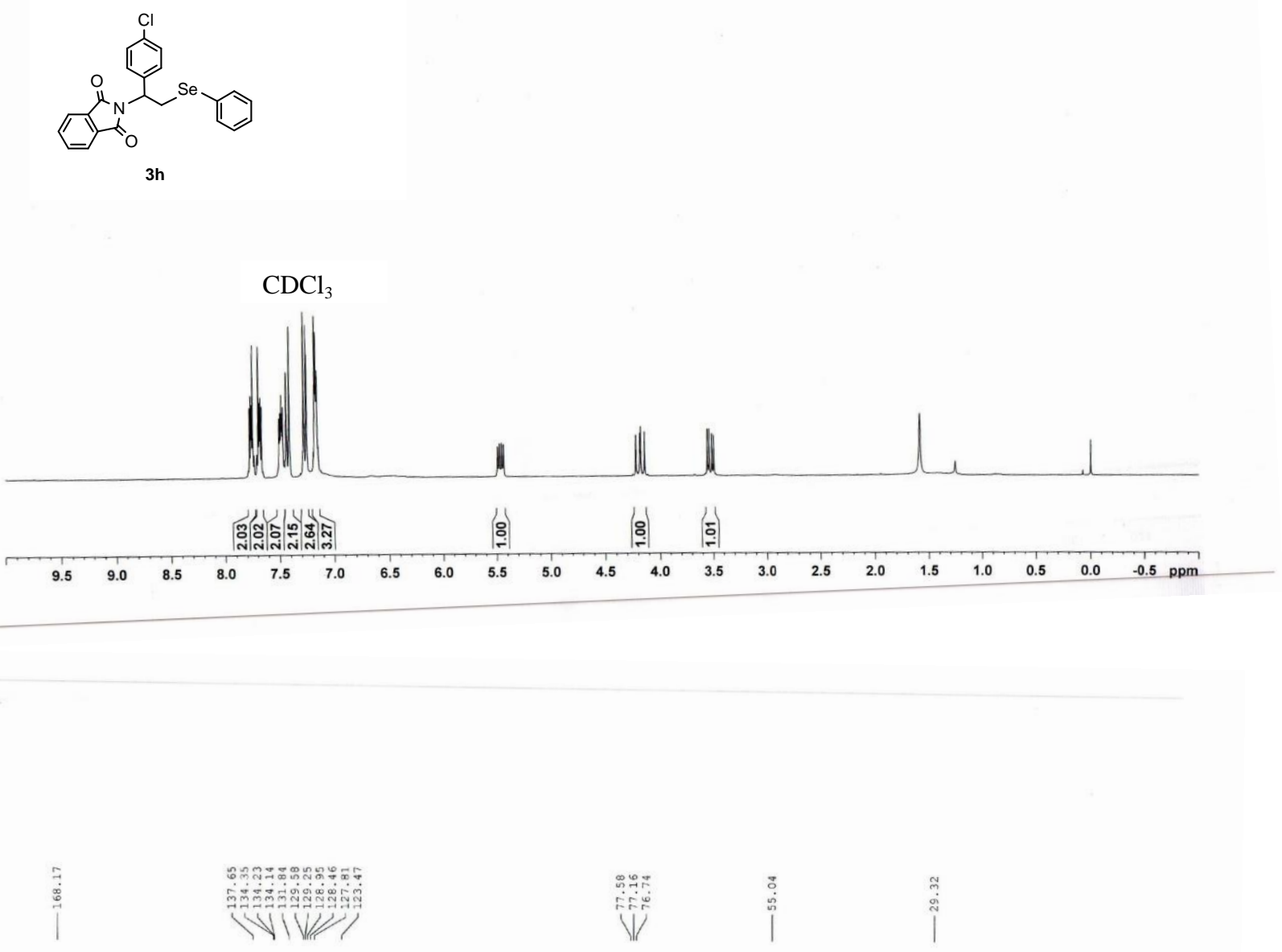

${ }^{13} \mathrm{C}$ NMR $\left(75 \mathrm{MHz}, \mathrm{CDCl}_{3}\right)$

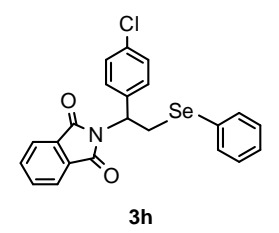

Sh

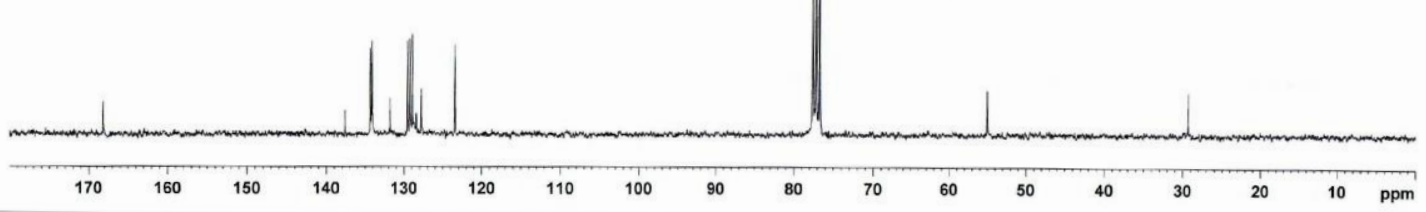

S29 

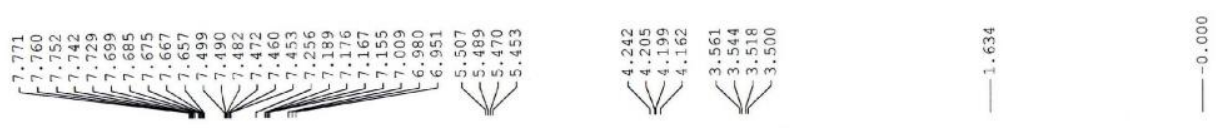

${ }^{1} \mathrm{H}$ NMR (300 MHz, $\mathrm{CDCl}_{3}$ )
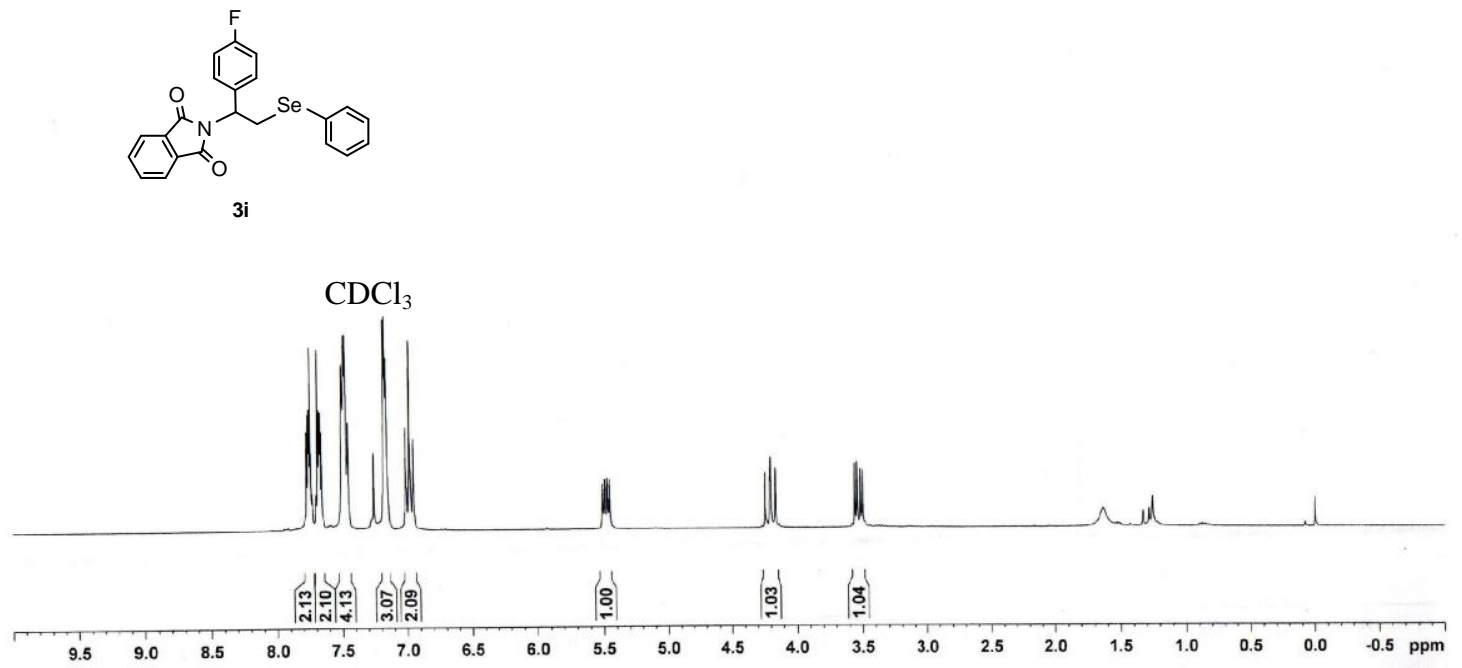

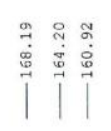
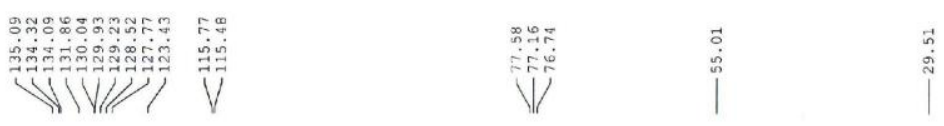

${ }^{13} \mathrm{C}$ NMR $\left(75 \mathrm{MHz}, \mathrm{CDCl}_{3}\right)$

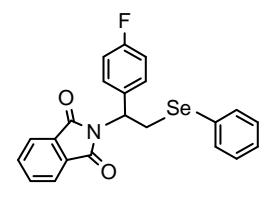

$3 \mathbf{i}$

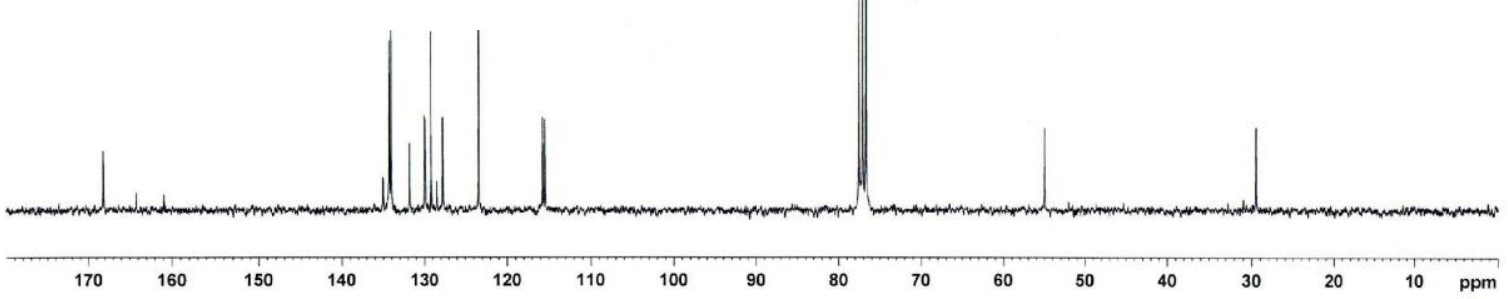

S30 

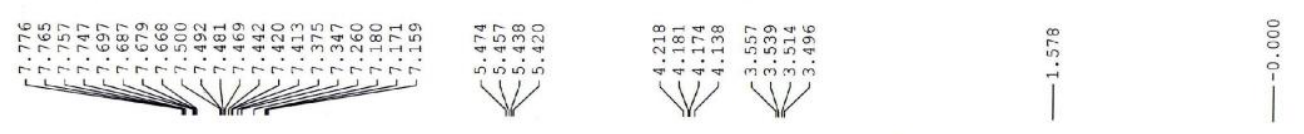

${ }^{1} \mathrm{H}$ NMR (300 MHz, $\mathrm{CDCl}_{3}$ )
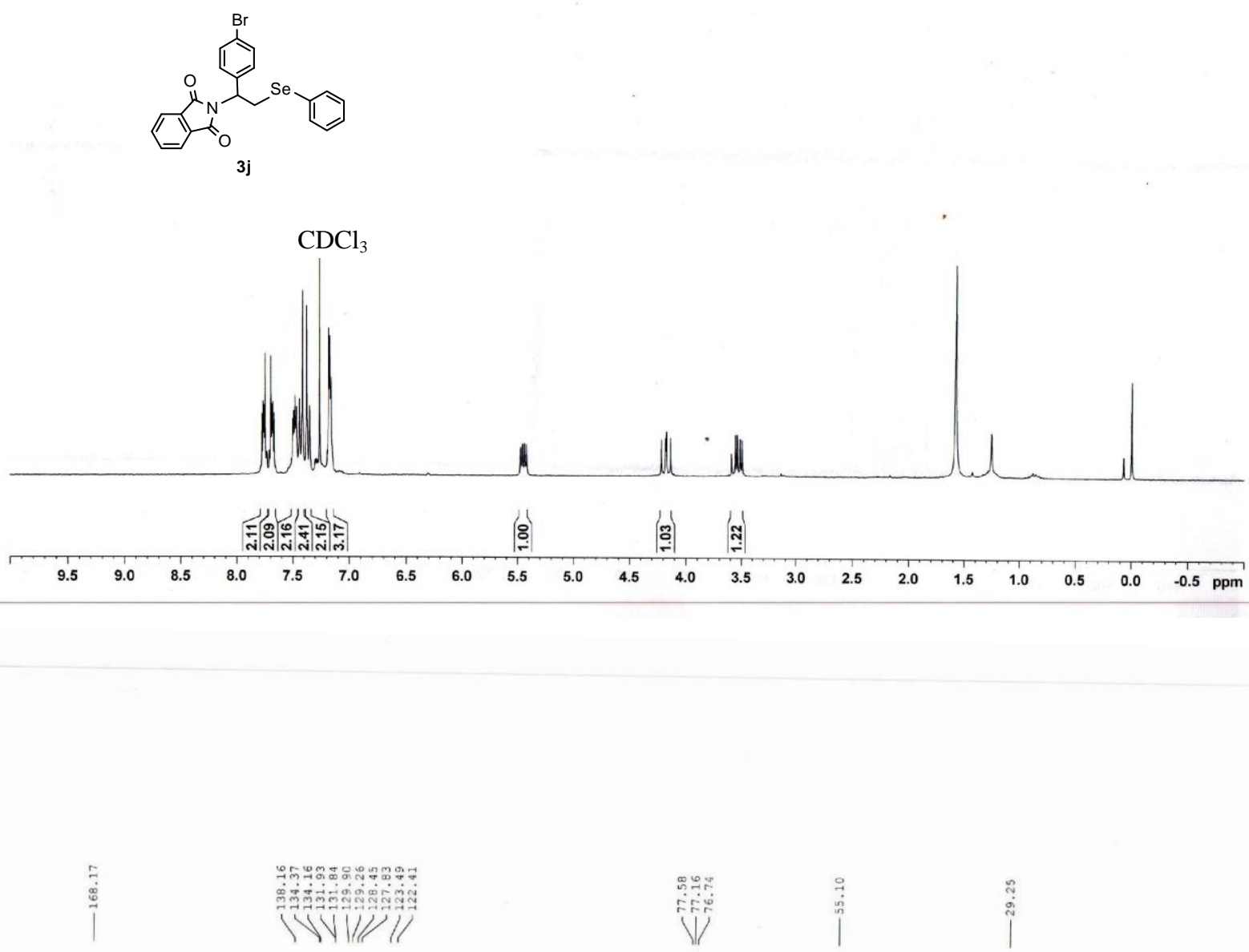

${ }^{13} \mathrm{C}$ NMR $\left(75 \mathrm{MHz}, \mathrm{CDCl}_{3}\right.$ )
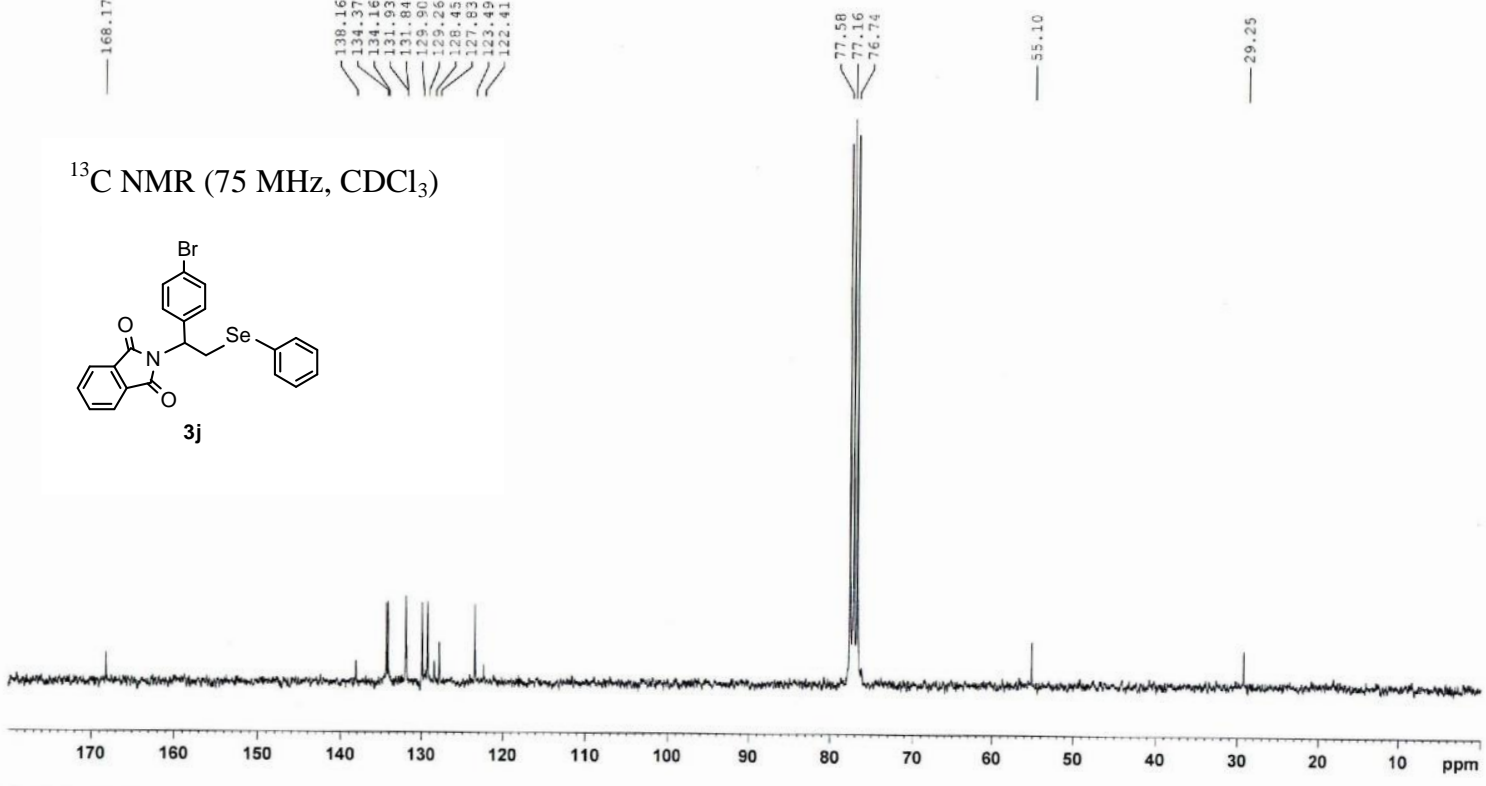

S31 
${ }^{1} \mathrm{H}$ NMR $\left(300 \mathrm{MHz}, \mathrm{CDCl}_{3}\right)$

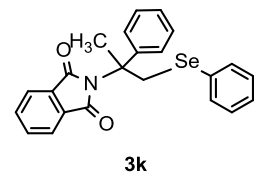

3k

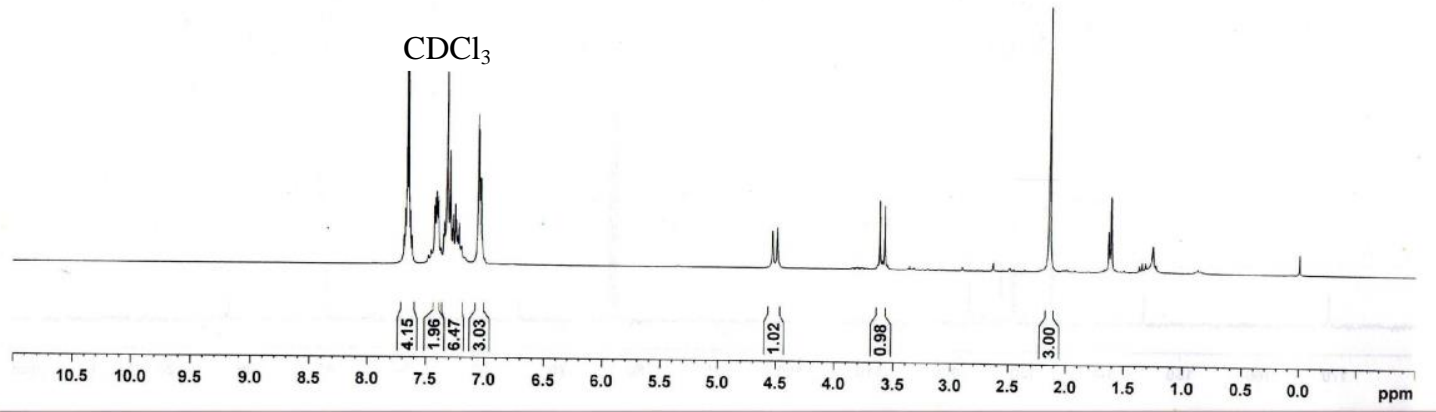

|

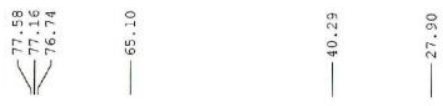

${ }^{13} \mathrm{C}$ NMR $\left(75 \mathrm{MHz}, \mathrm{CDCl}_{3}\right)$

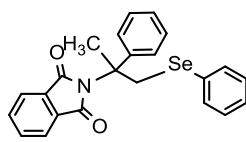

3k

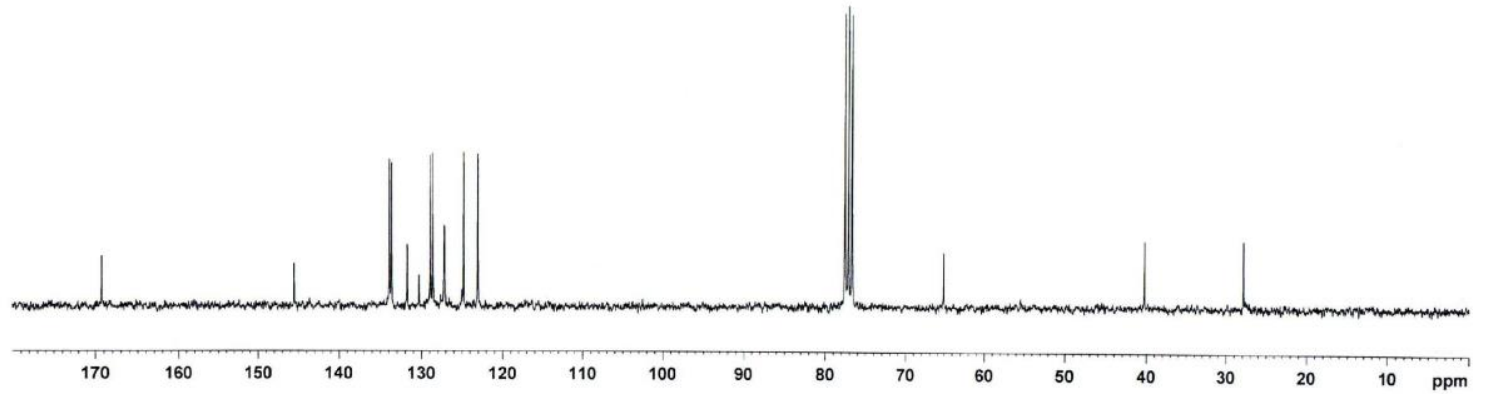




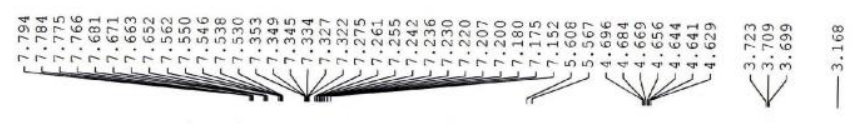

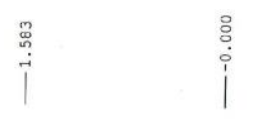

${ }^{1} \mathrm{H} \mathrm{NMR}\left(300 \mathrm{MHz}, \mathrm{CDCl}_{3}\right)$

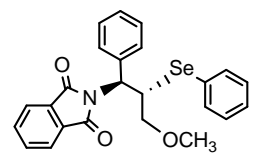

$3 m$
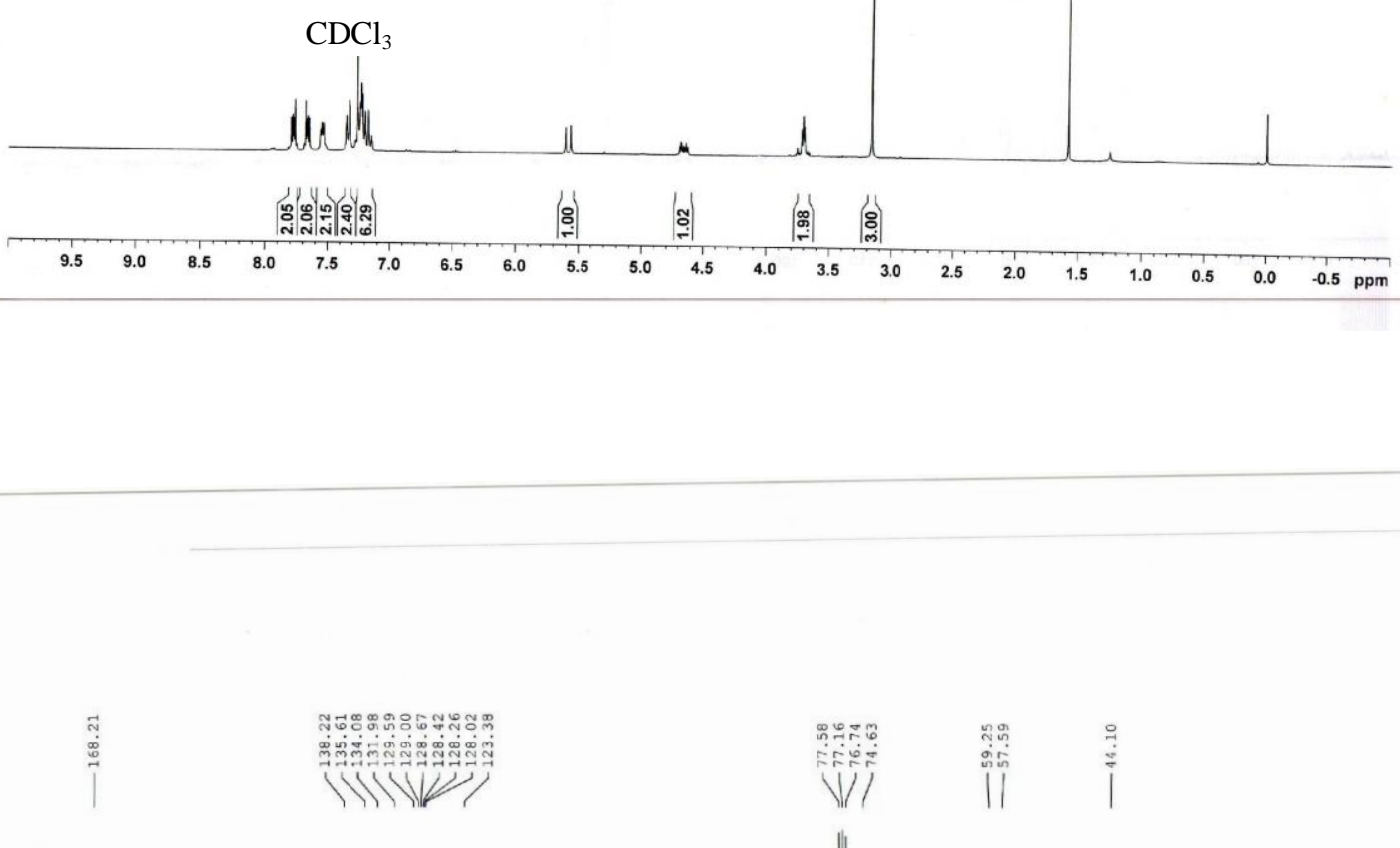

${ }^{13} \mathrm{C}$ NMR $\left(75 \mathrm{MHz}, \mathrm{CDCl}_{3}\right)$

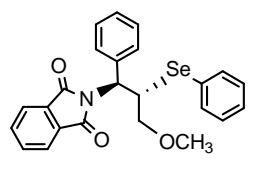

$3 m$

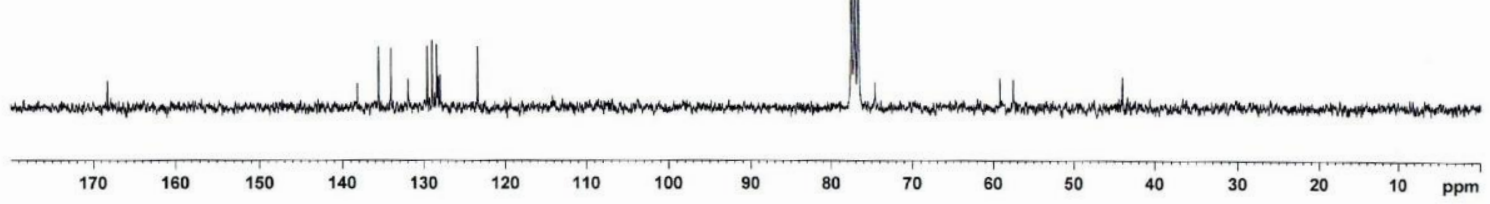

S33 

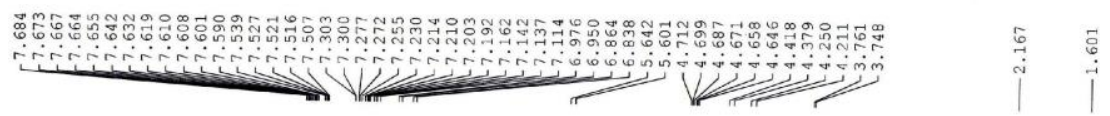

!

${ }^{1} \mathrm{H}$ NMR (300 MHz, $\mathrm{CDCl}_{3}$ )

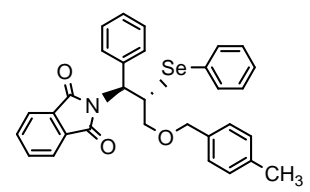

Bn
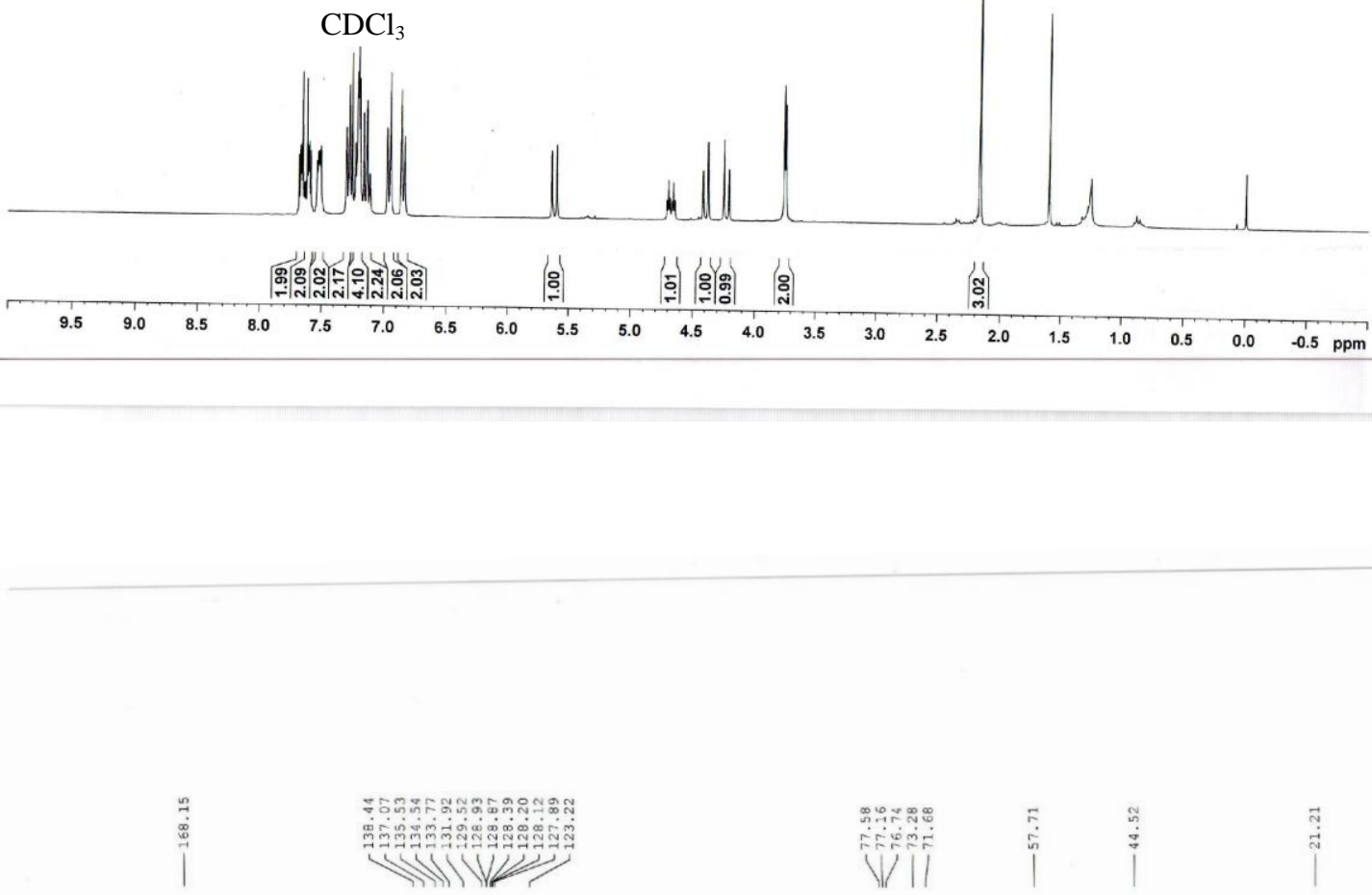

${ }^{13} \mathrm{C}$ NMR $\left(75 \mathrm{MHz}, \mathrm{CDCl}_{3}\right)$

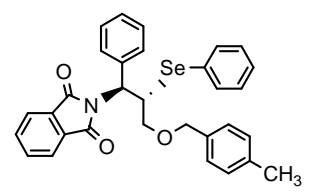

$3 n$
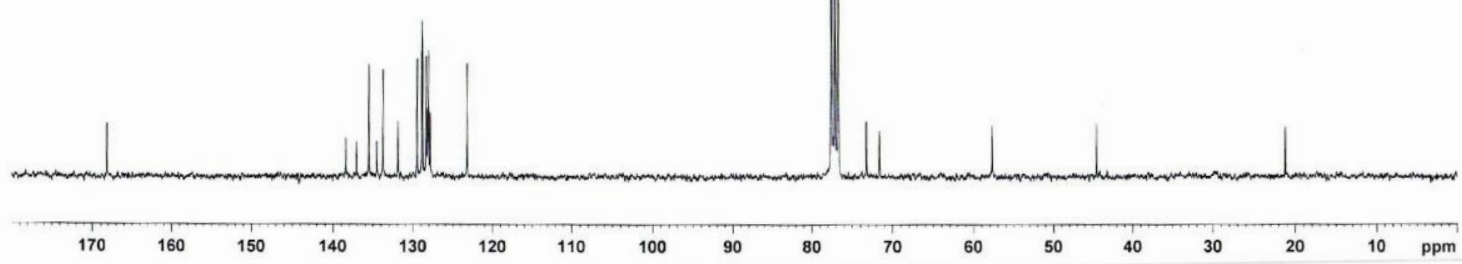

S34 
${ }^{1} \mathrm{H}$ NMR (300 MHz, $\left.\mathrm{CDCl}_{3}\right)$

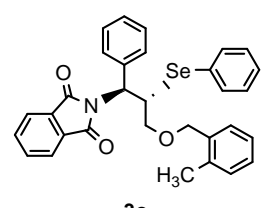

3o
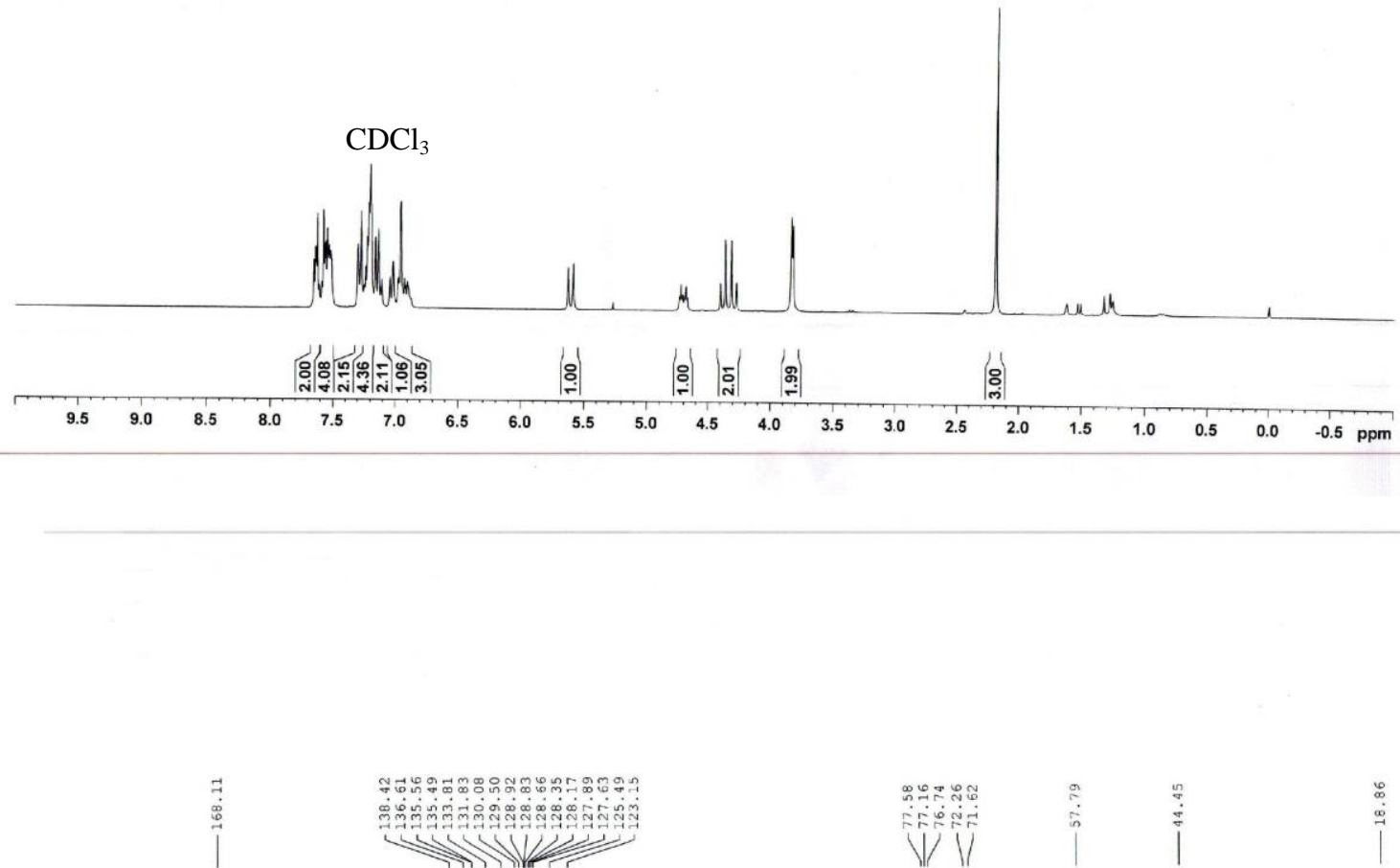

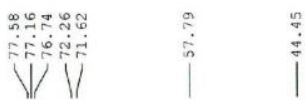

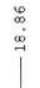

${ }^{13} \mathrm{C} \mathrm{NMR}\left(75 \mathrm{MHz}, \mathrm{CDCl}_{3}\right)$

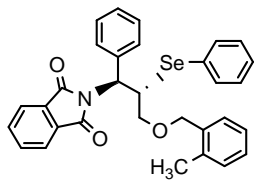

30

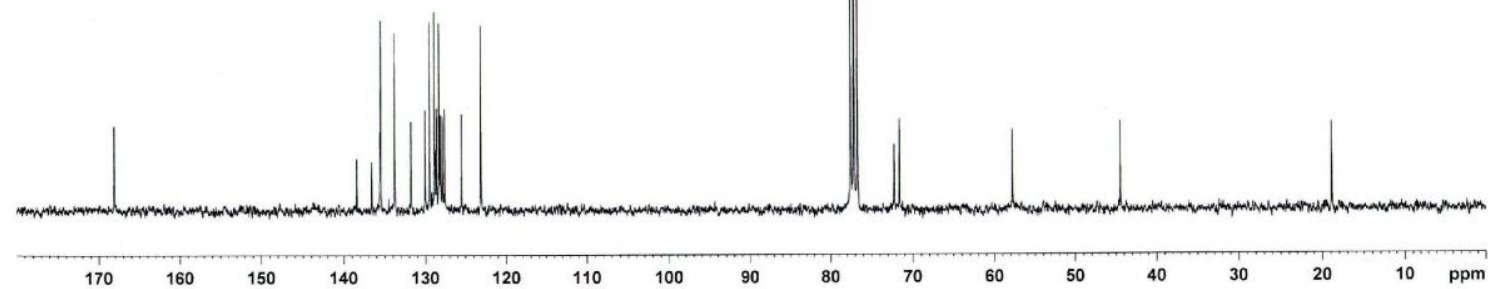



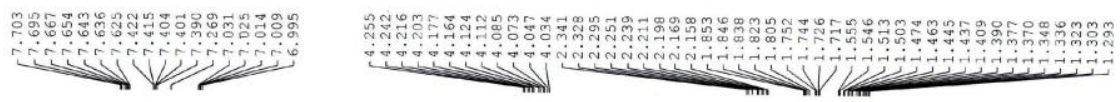

${ }^{1} \mathrm{H}$ NMR (300 MHz, $\mathrm{CDCl}_{3}$ )

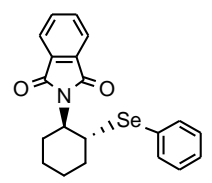

$3 p$
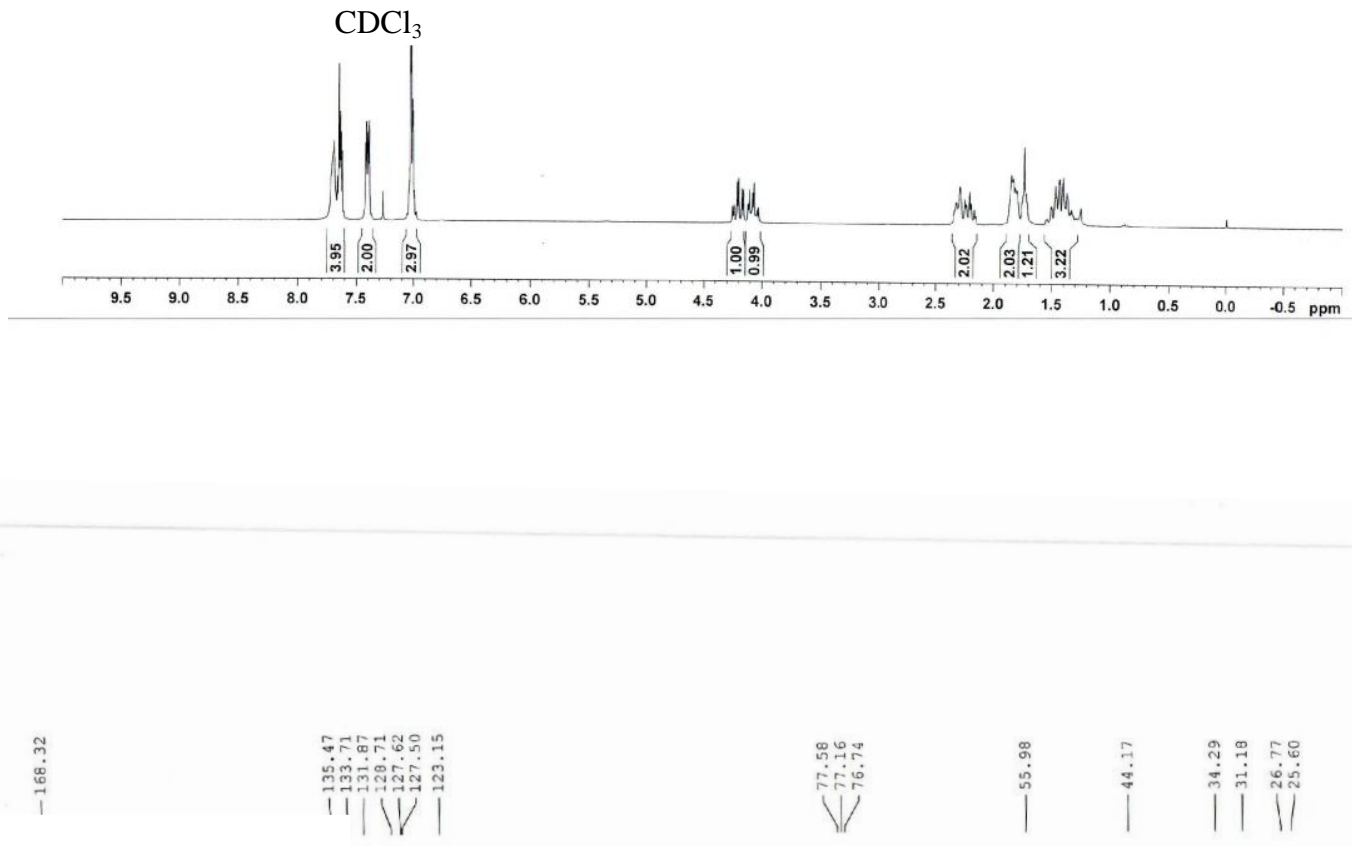

${ }^{13} \mathrm{C}$ NMR $\left(75 \mathrm{MHz}, \mathrm{CDCl}_{3}\right.$ )

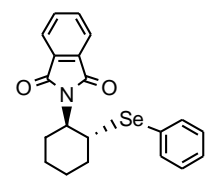

$3 p$

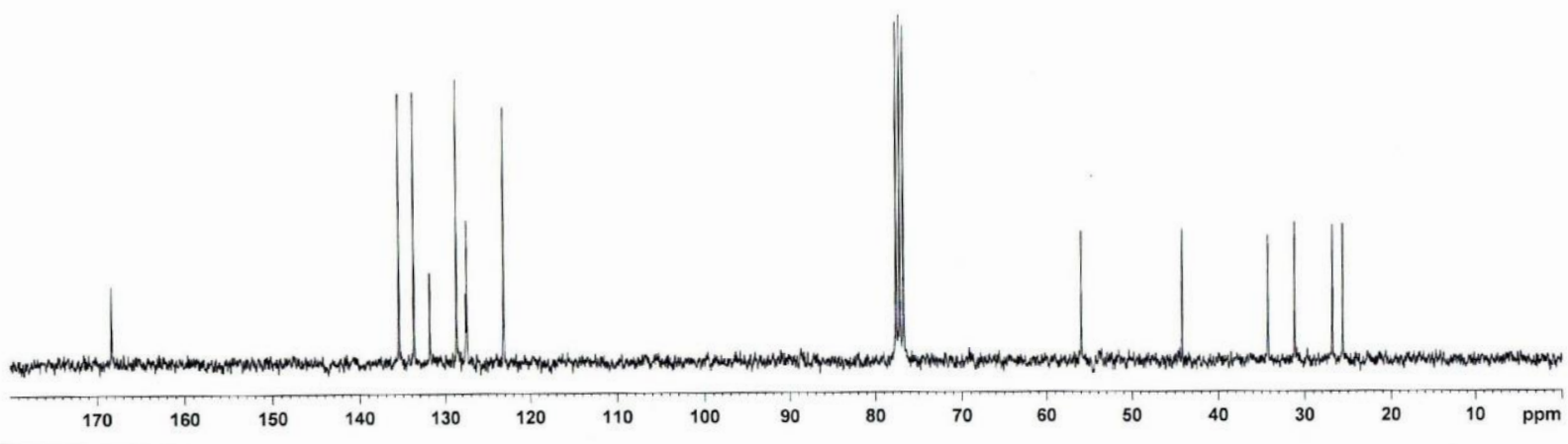

S36 
${ }^{1} \mathrm{H} \mathrm{NMR}\left(300 \mathrm{MHz}, \mathrm{CDCl}_{3}\right)$
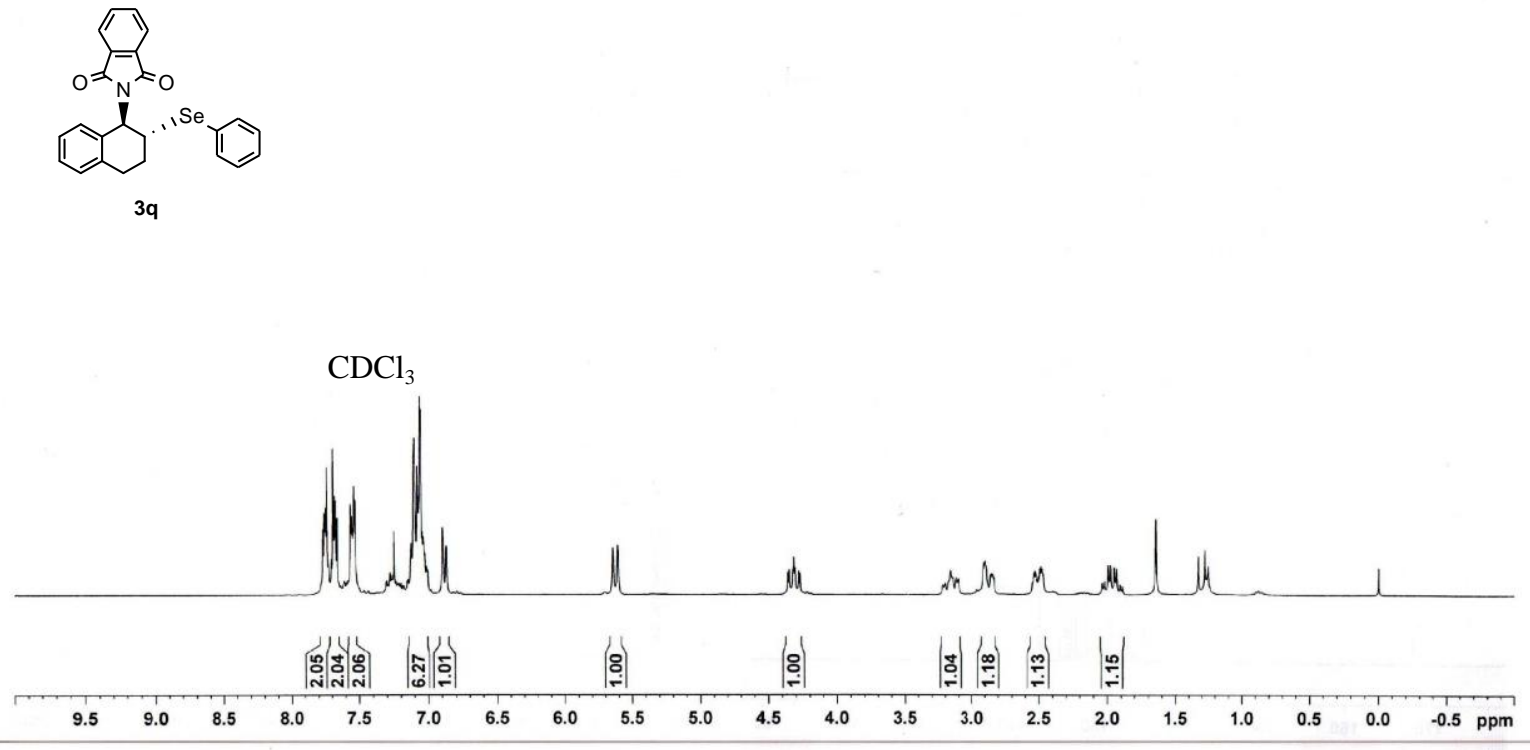

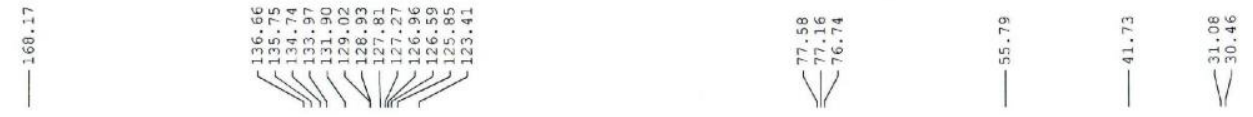

${ }^{13} \mathrm{C} \mathrm{NMR}\left(75 \mathrm{MHz}, \mathrm{CDCl}_{3}\right)$

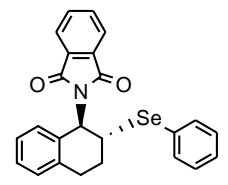

$3 q$

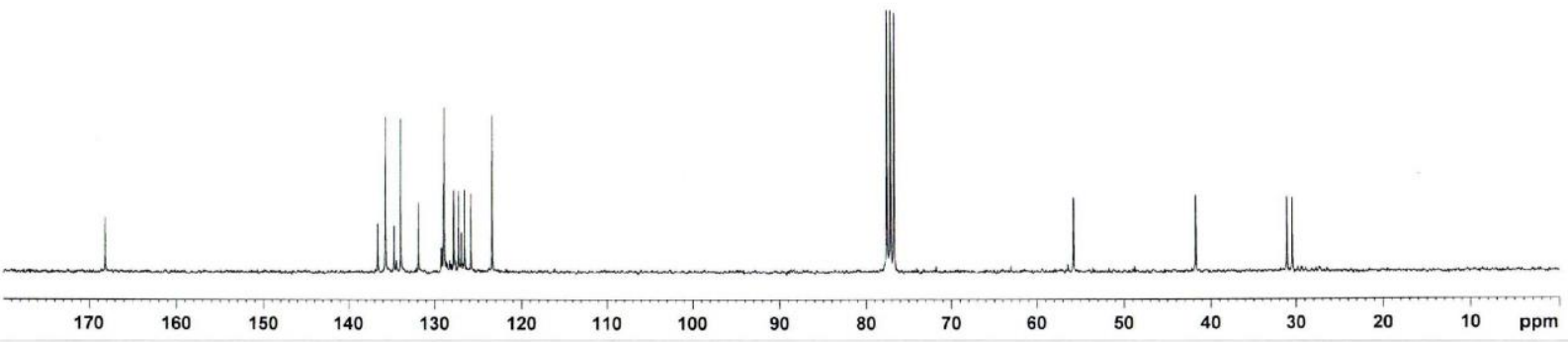




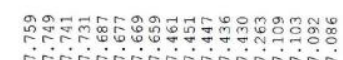

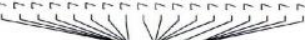

${ }^{1} \mathrm{H}$ NMR (300 MHz, $\mathrm{CDCl}_{3}$ )

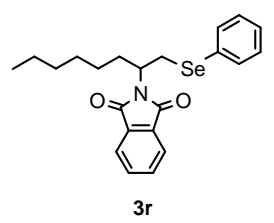

$\mathrm{CDCl}_{3}$

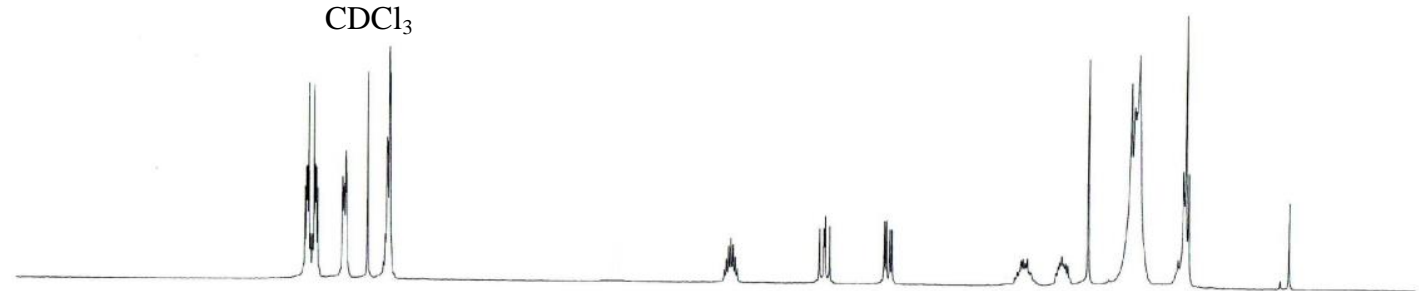

ถู่

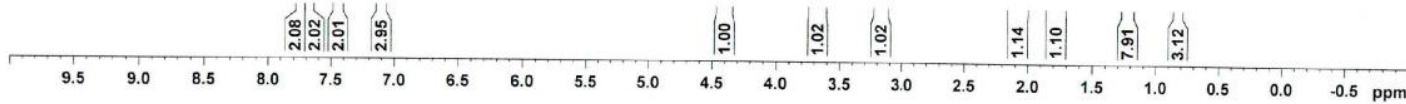

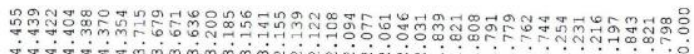

-

|

${ }^{13} \mathrm{C}$ NMR $\left(75 \mathrm{MHz}, \mathrm{CDCl}_{3}\right)$
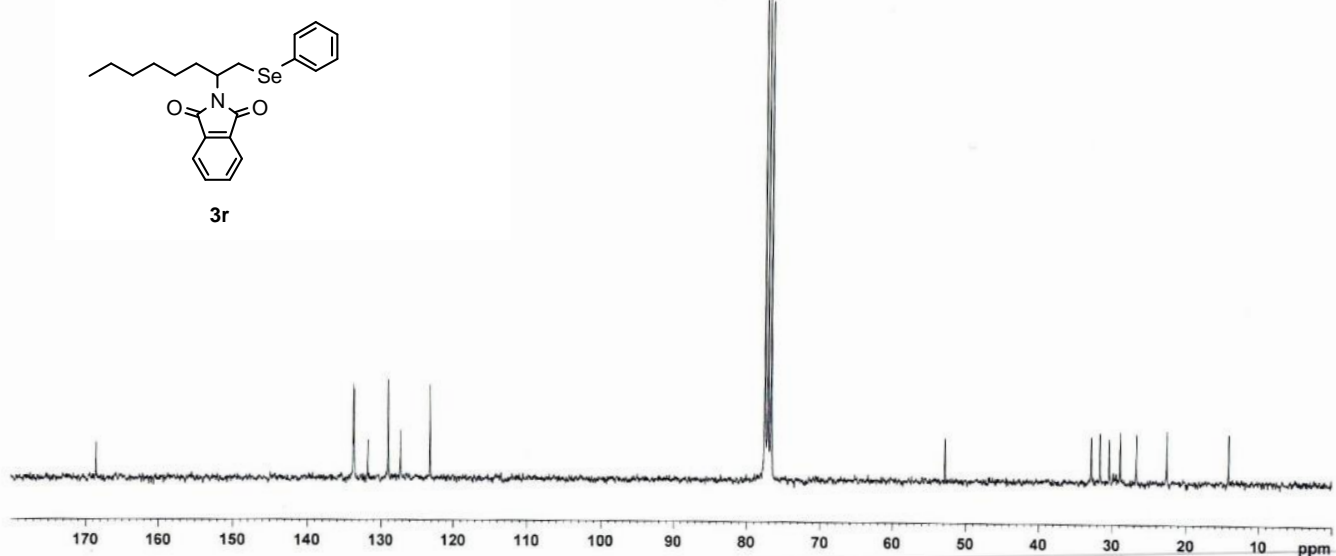
The HMBC spectrum of compound $\mathbf{3 r}\left(400 \mathrm{MHz}, \mathrm{CDCl}_{3}\right)$ :

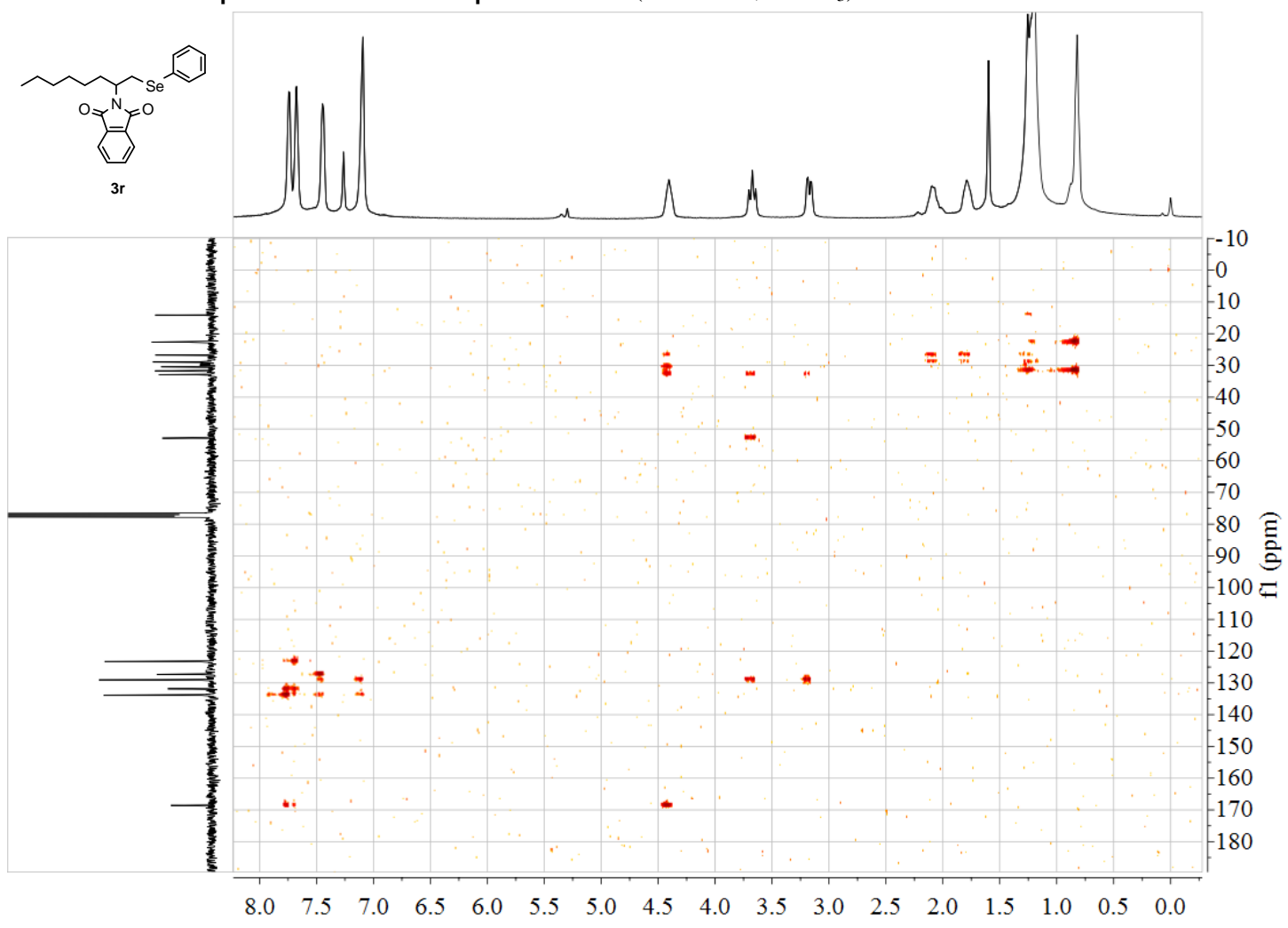

The HSQC spectrum of compound $\mathbf{3 r}\left(400 \mathrm{MHz}, \mathrm{CDCl}_{3}\right)$ :

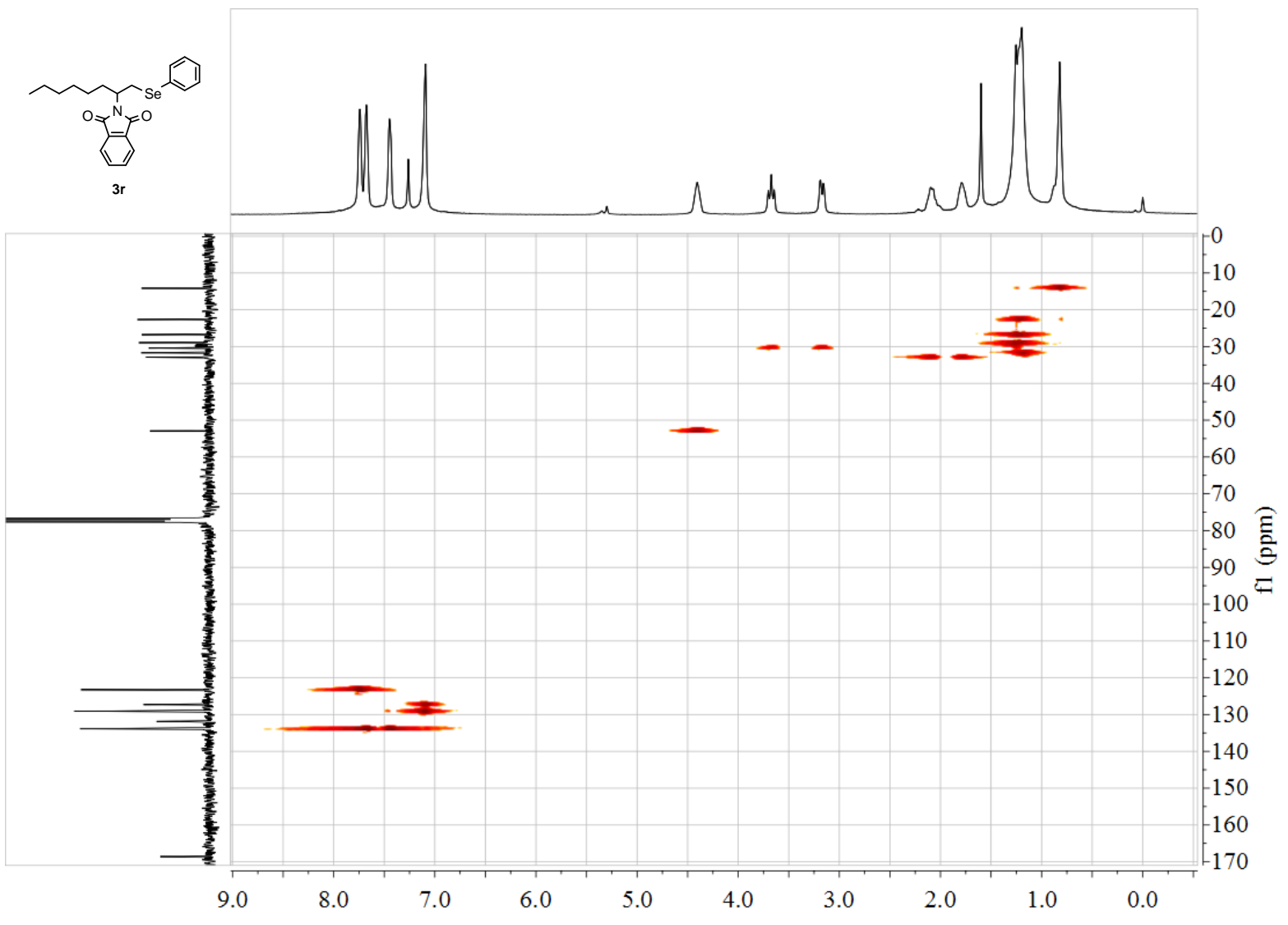




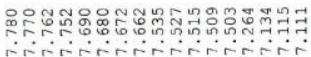

inivin

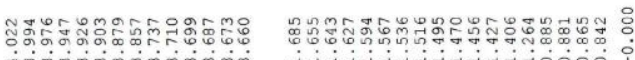

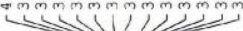

sher

${ }^{1} \mathrm{H}$ NMR $\left(300 \mathrm{MHz}, \mathrm{CDCl}_{3}\right)$
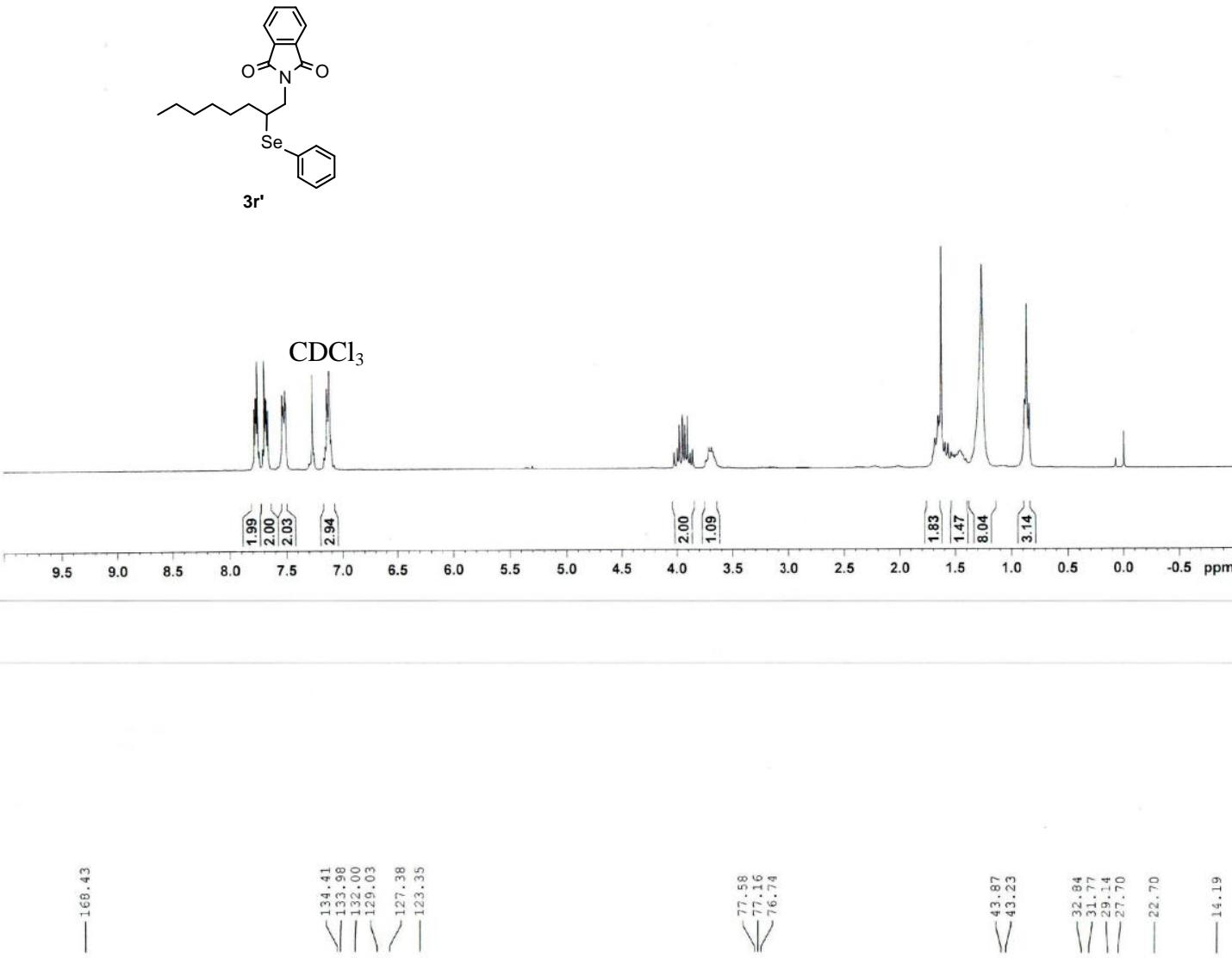

${ }^{13} \mathrm{C}$ NMR (75 MHz, $\mathrm{CDCl}_{3}$ )
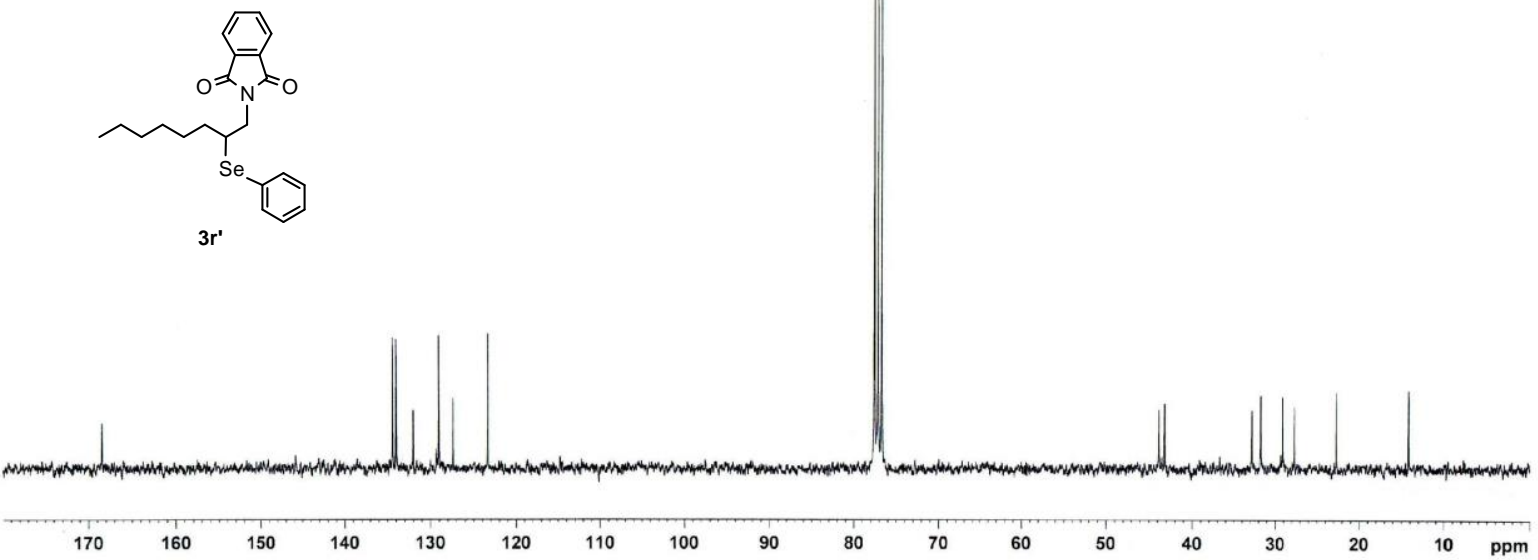


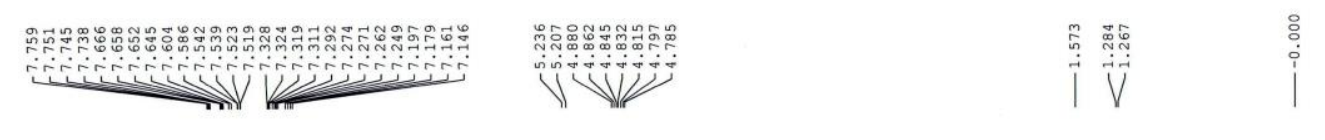

${ }^{1} \mathrm{H}$ NMR $\left(400 \mathrm{MHz}, \mathrm{CDCl}_{3}\right)$

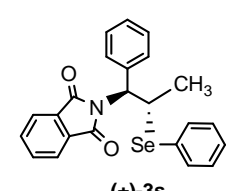

$( \pm)-3 s$

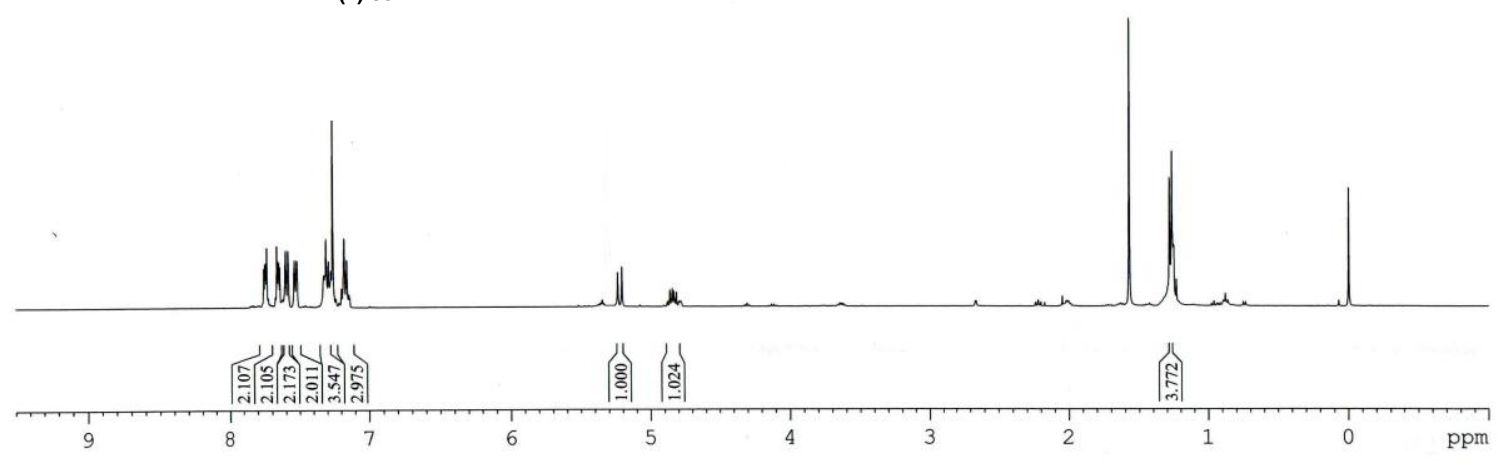

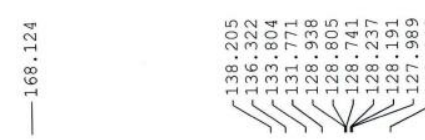

${ }^{13} \mathrm{C}$ NMR $\left(100 \mathrm{MHz}, \mathrm{CDCl}_{3}\right)$

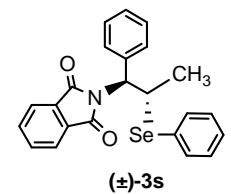

$( \pm)-3 s$

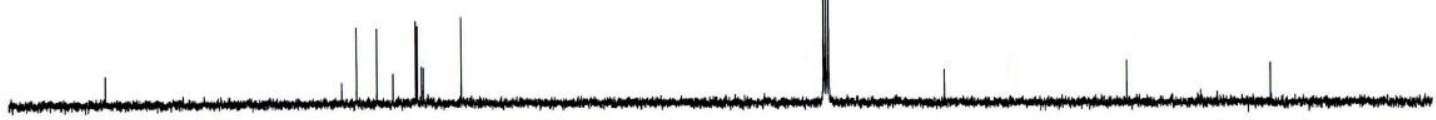

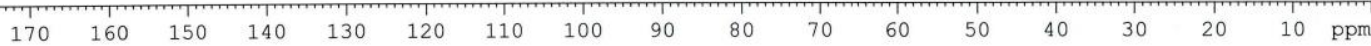



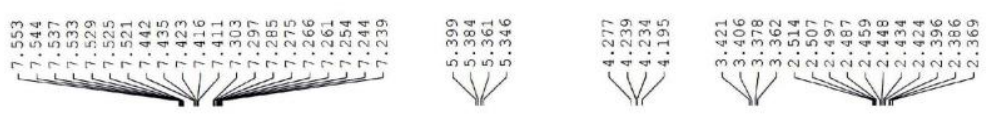

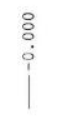

${ }^{1} \mathrm{H}$ NMR (300 MHz, $\mathrm{CDCl}_{3}$ )
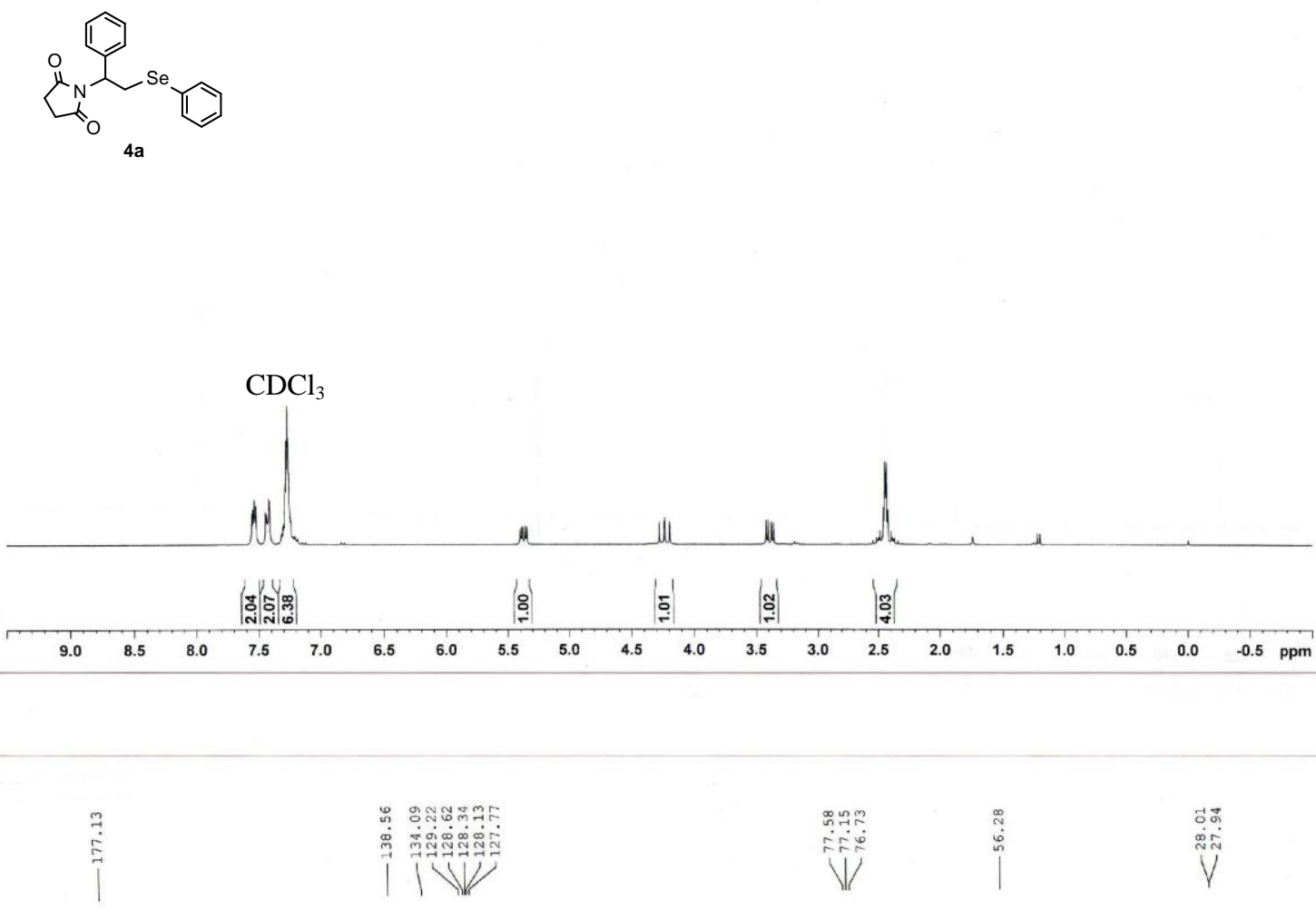

${ }^{13} \mathrm{C}$ NMR $\left(75 \mathrm{MHz}, \mathrm{CDCl}_{3}\right)$
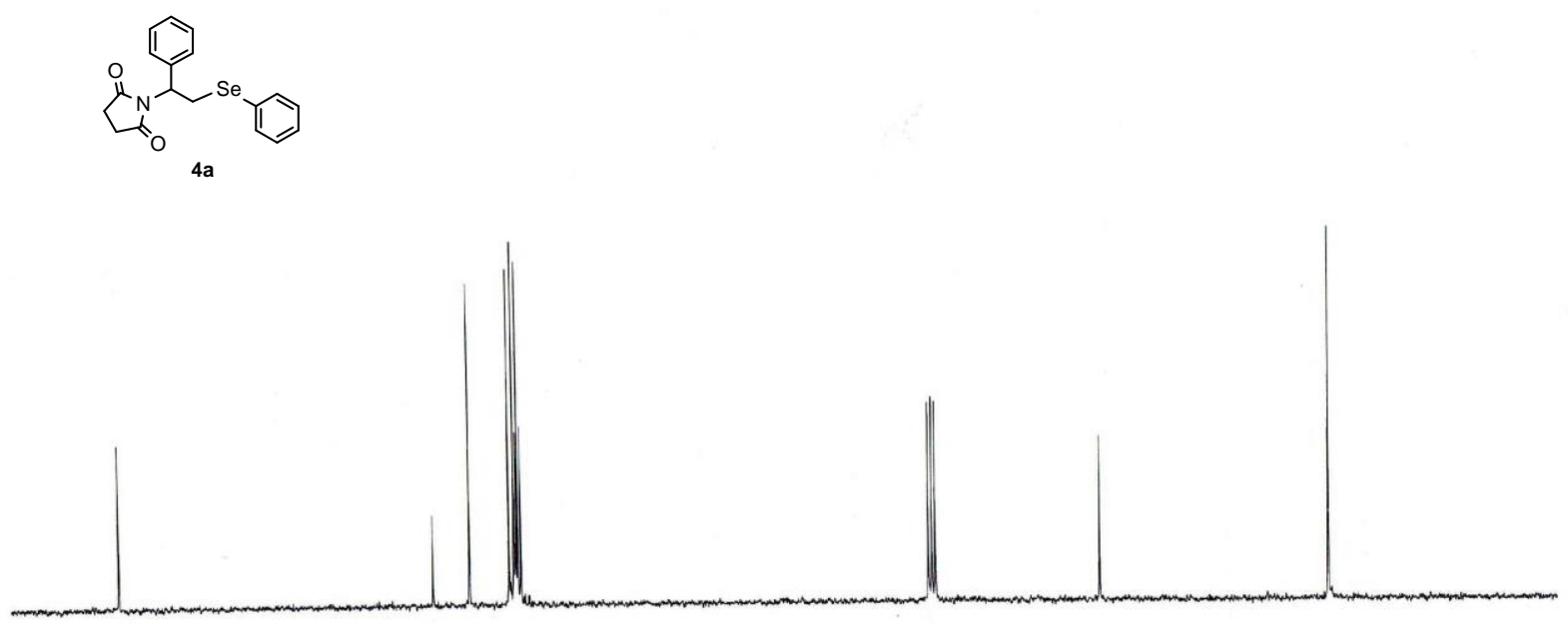

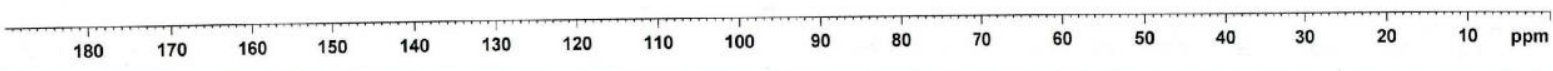

S42 


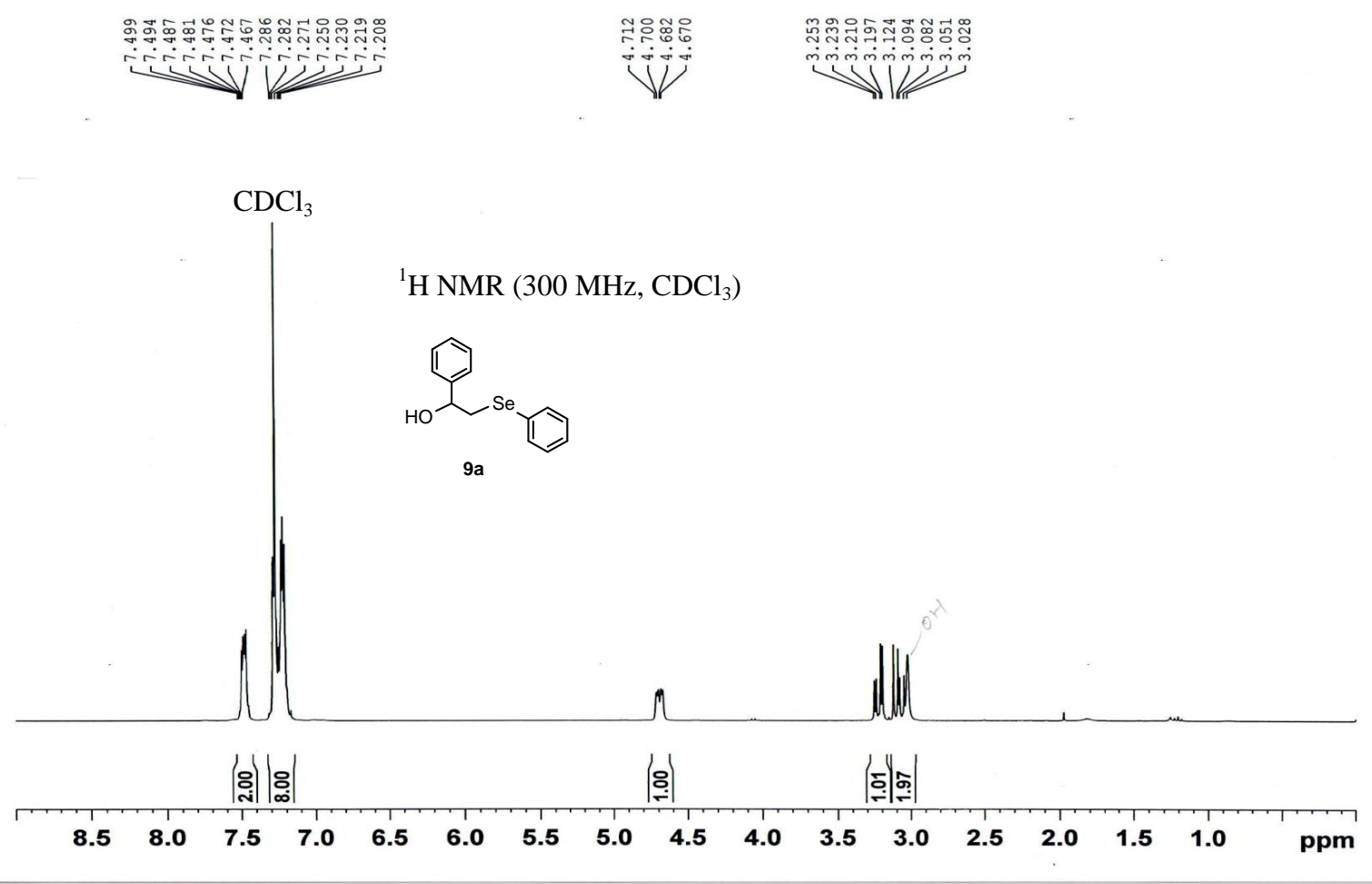

${ }^{13} \mathrm{C}$ NMR $\left(75 \mathrm{MHz}, \mathrm{CDCl}_{3}\right.$ )

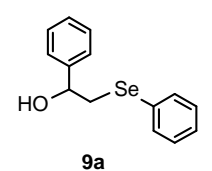

$9 a$

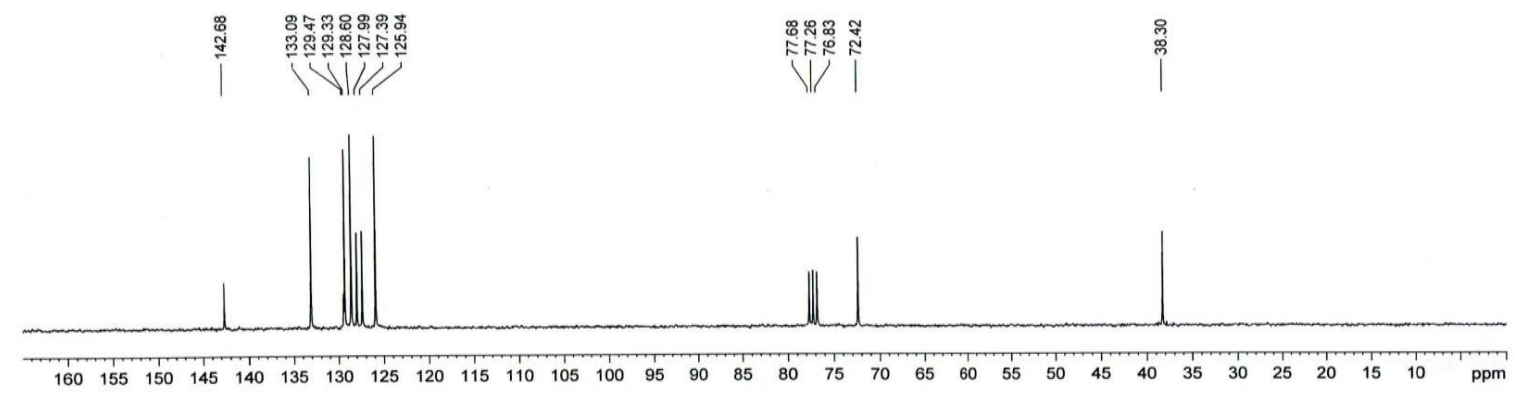



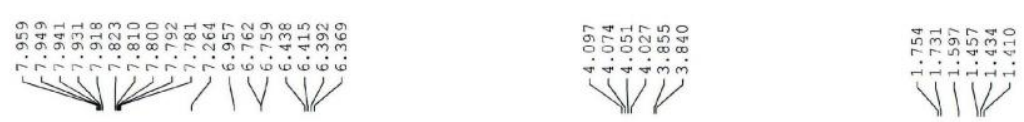

i

${ }^{1} \mathrm{H}$ NMR $\left(300 \mathrm{MHz}, \mathrm{CDCl}_{3}\right.$ )

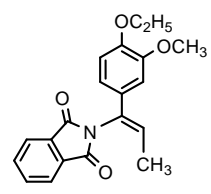

$5 a$

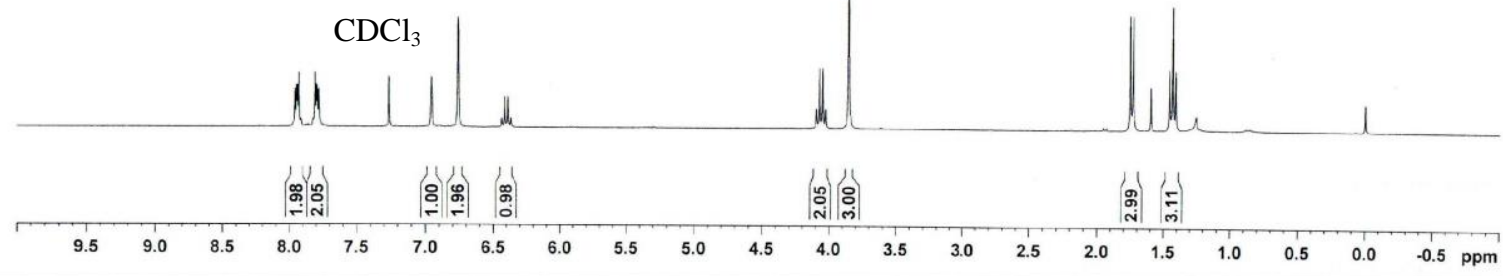

| |

${ }^{13} \mathrm{C} \mathrm{NMR}\left(75 \mathrm{MHz}, \mathrm{CDCl}_{3}\right)$

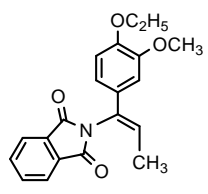

$5 a$

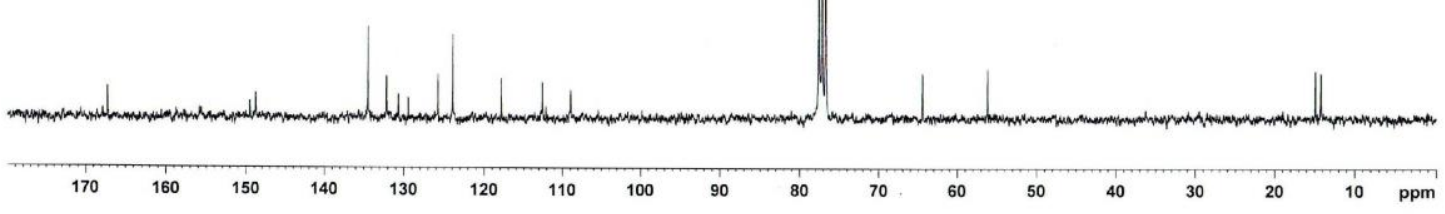

S44 
${ }^{1} \mathrm{H}$ NMR $\left(300 \mathrm{MHz}, \mathrm{CDCl}_{3}\right)$

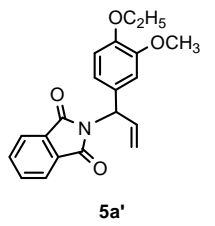

$5 a^{\prime}$

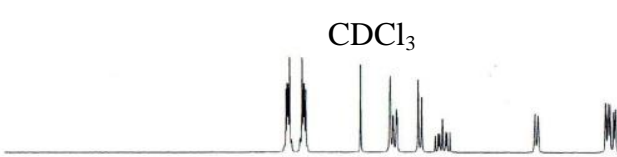

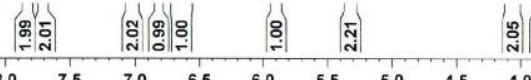

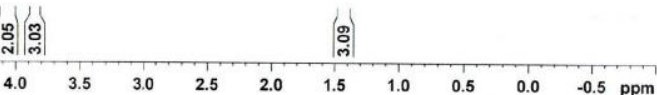

|

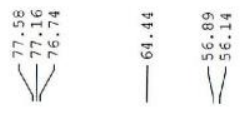

${ }^{13} \mathrm{C}$ NMR $\left(75 \mathrm{MHz}, \mathrm{CDCl}_{3}\right)$

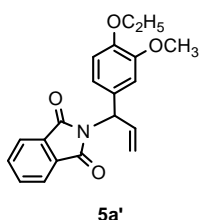

$5 a^{\prime}$

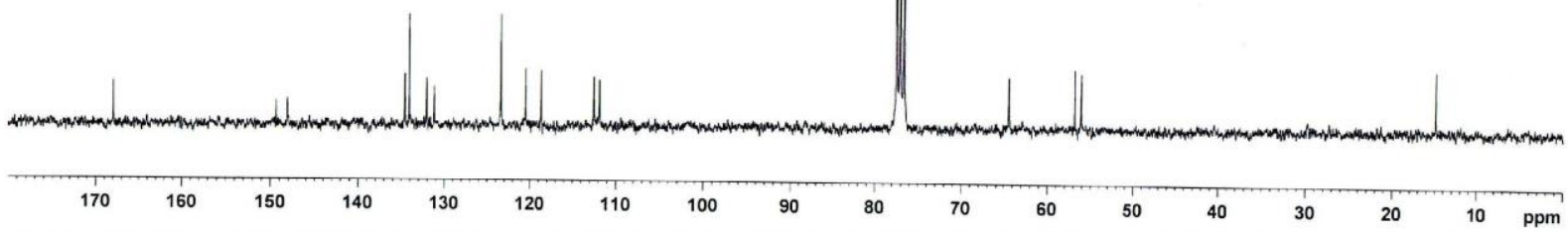




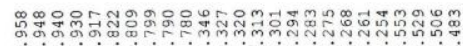

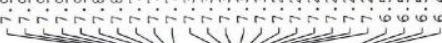

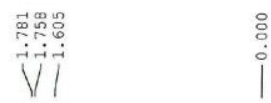

${ }^{1} \mathrm{H}$ NMR (300 MHz, $\left.\mathrm{CDCl}_{3}\right)$

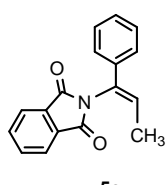

$5 c$

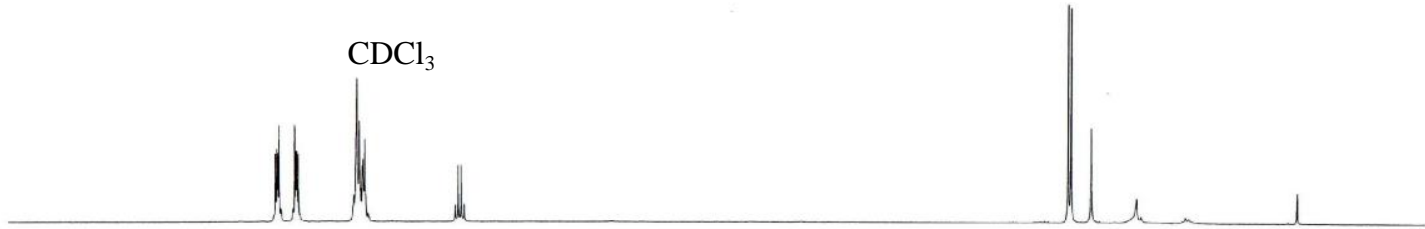

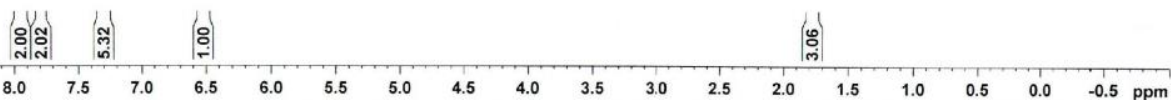
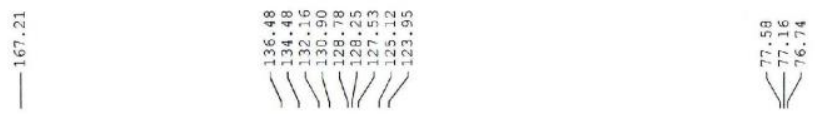

${ }^{13} \mathrm{C}$ NMR $\left(75 \mathrm{MHz}, \mathrm{CDCl}_{3}\right)$

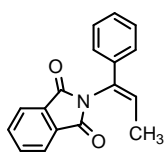

$5 c$

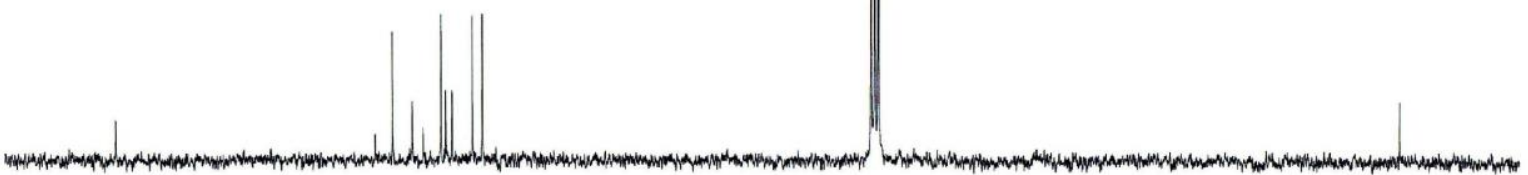

$\begin{array}{llllllllllllllllll}170 & 160 & 150 & 140 & 130 & 120 & 110 & 100 & 90 & 80 & 70 & 60 & 50 & 40 & 30 & 20 & 10 & 0\end{array}$ 
${ }^{1} \mathrm{H}$ NMR $\left(400 \mathrm{MHz}, \mathrm{CDCl}_{3}\right)$
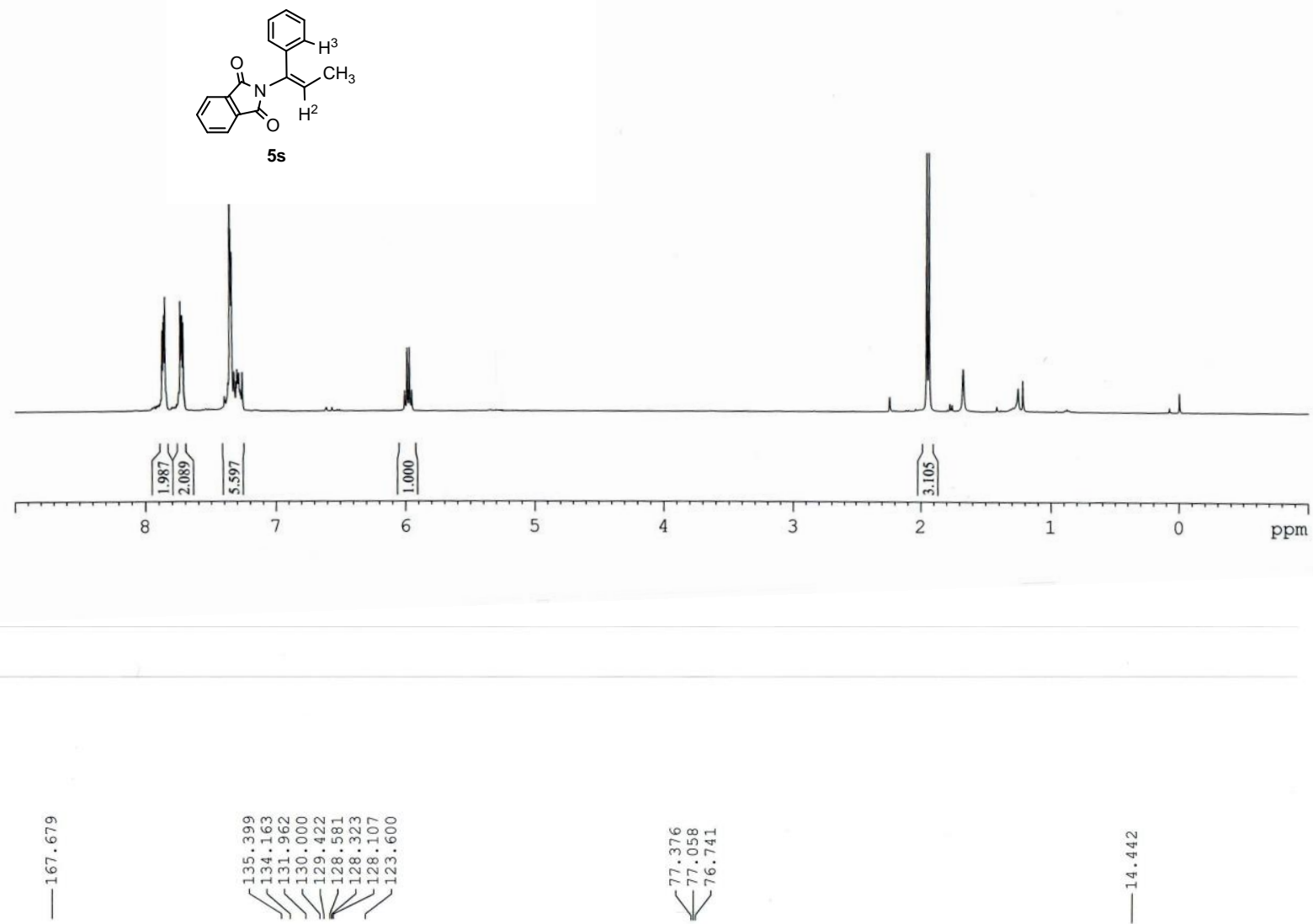

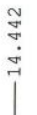

${ }^{13} \mathrm{C}$ NMR $\left(100 \mathrm{MHz}, \mathrm{CDCl}_{3}\right)$
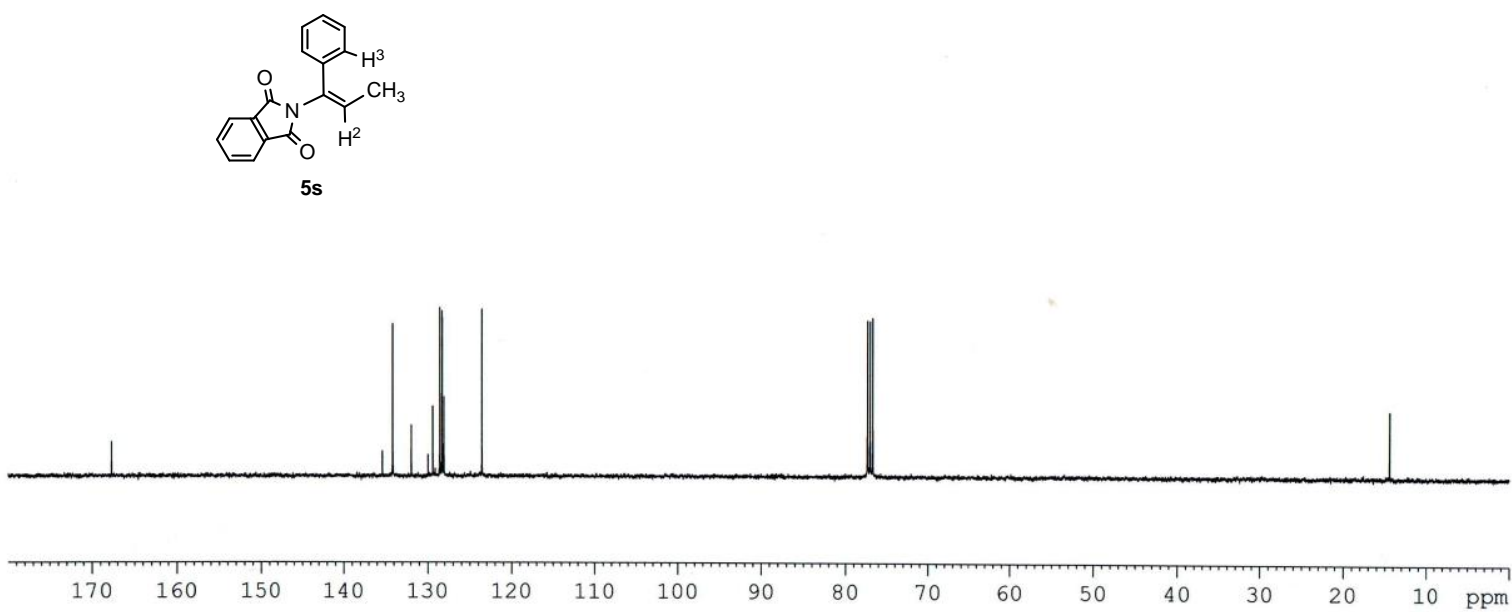
Copies of ROESY Spectra for compounds $5 \mathrm{c}$ and $5 \mathrm{~s}$

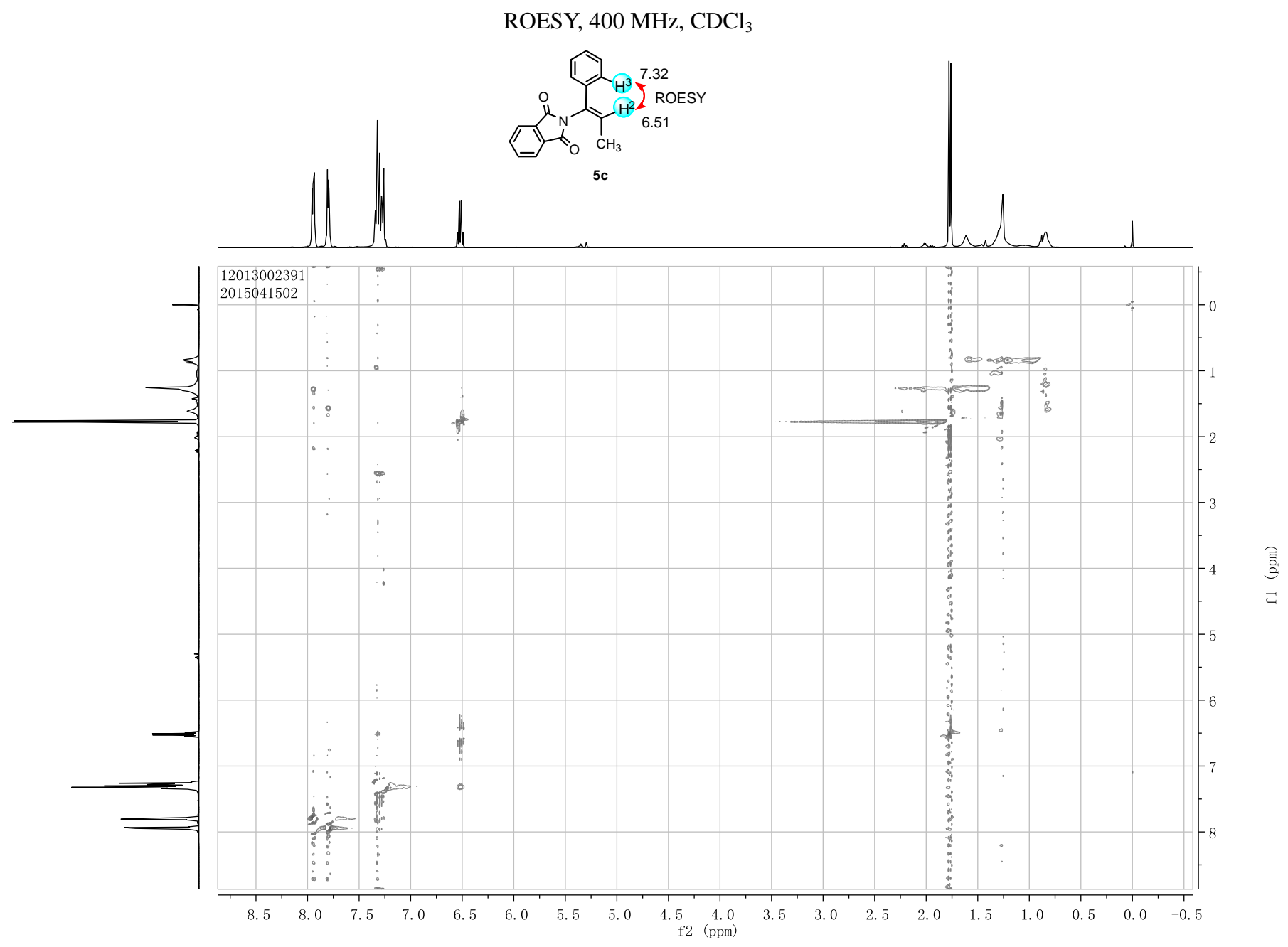

S48 
ROESY, $400 \mathrm{MHz}, \mathrm{CDCl}_{3}$

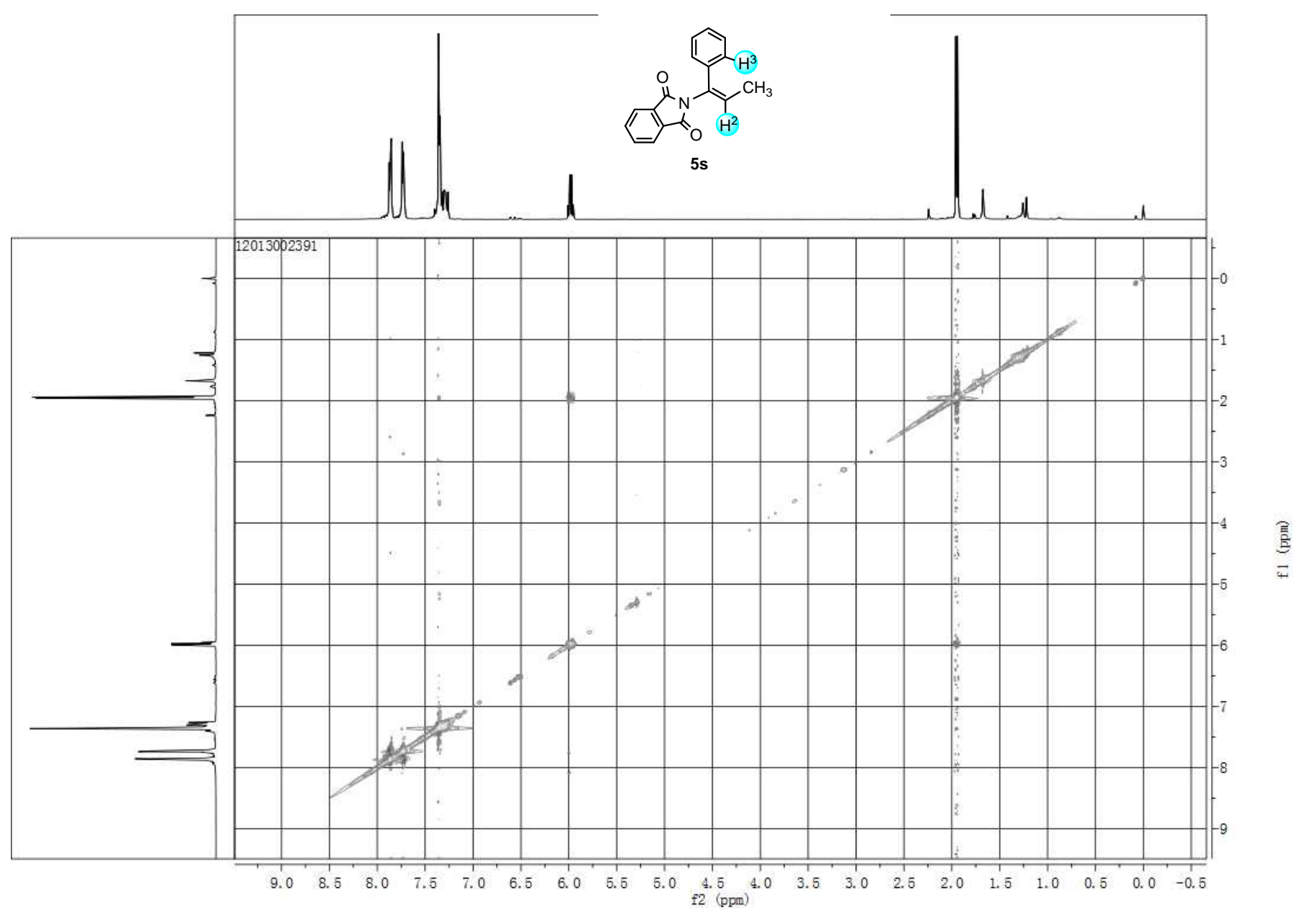

